\title{
BROADBAND ABSOLUTE ABSORPTION MEASUREMENTS OF ATMOSPHERIC CONTINUA WITH MILLIMETER WAVE CAVITY RINGDOWN SPECTROSCOPY
}

\author{
DISSERTATION \\ Presented in Partial Fulfillment of the Requirements for \\ the Degree Doctor of Philosophy in the Graduate \\ School of The Ohio State University
}

By

Andrey I. Meshkov, B.S.

$* * * * *$

The Ohio State University

2006

Dissertation Committee:

Approved by

Professor Frank C. De Lucia, Adviser

Professor Eric Herbst

Professor Thomas Humanic

Professor Douglas Schumacher

Adviser

Graduate Program in Physics 


\begin{abstract}
It has been known for more than sixty years that all of the atmospheric absorption can not be accounted for by the standard lineshape theory. Remarkably, to this day there is no consensus on the physical origin of the "excess" absorption, called an atmospheric continuum. This is certainly due in no small part to the lack of accurate laboratory measurements, performed for a wide range of pressures, temperatures and frequencies. Unlike in low pressure gas phase spectroscopy where rapid change in the narrow lines absorption is used to remove the slowly varying baseline, quantification of slowly varying with frequency atmospheric absorption is much more difficult.

In this thesis we will describe the instrument for the measurement of continua and other slowly varying absorption phenomena in the millimeter and submillimeter (a.k.a. terahertz) spectral region. By replacing the traditional frequency domain phase lock measurement of cavity $\mathrm{Q}$ with the time domain cavity ringdown this instrument can measure continua and broad line absorptions at $\sim 6000$ distinct cavity frequencies from 170 to $260 \mathrm{GHz}$ in $\sim 3$ seconds. These near simultaneous measurements can include both regions dominated by contributions from near line center effects and other regions where "continua" effects are important. The result is
\end{abstract}


a data set that contains, without the use of parameters from other experiments, all of the required experimental information. This approach significantly reduces systematic effects and makes possible more realistic assessment of errors.

The instrument was used to study the absorption of nitrogen, oxygen, dry and moist air at a number of temperatures in -40 to $+70{ }^{\circ} \mathrm{C}$ range and pressures from 0 to 3 atmospheres. Results of the measurements were compared with current absorption models and previous laboratory measurements. In addition, we have used the developed technique to study the pressure broadening and pressure shift temperature behavior of the $183.310 \mathrm{GHz}$ water line, important in the atmospheric remote sensing. 
Dedicated to my parents 


\section{ACKNOWLEDGEMENTS}

In science, as in life, a person can not truly excel in anything without the help of others, whether it comes in a form of sharing knowledge, giving an advice or providing moral support. I am deeply thankful to all the people I had a privilege to work and interact with during my graduate work at the Ohio State University. All your assistance is appreciated and valued greatly.

Of people I want to thank personally, the first is my thesis advisor, Dr. Frank C. De Lucia. This thesis would not be possible without his comprehensive scientific knowledge, vast experience, amazing enthusiasm and ability to always look at things positively.

I am grateful to all the members of the Microwave group for the exchange of ideas, help and friendship.

The help of Eric Weingartner in building the instrument support and the insulation was very much appreciated.

I would like to thank the Physics Department technical support staff for making my experimental research so much easier. Machine shop help was invaluable as many parts of the experiment were made there. Tom Kelch from the graduate student shop was always there for me if I needed to quickly make something myself. Bob Merritt kept the helium flowing and the computer facility staff made sure the right software was there when needed. I am thankful to Kent Ludwig from the low 
temperature shop for his help in designing the liquid nitrogen cooling system. A special thank you goes to Dr. John Hoftiezer from the electronics support for developing the electronic components, critical for the success of the experiment.

The love and understanding of my parents was felt even from beyond the ocean and has helped me to stay the course.

Finally, I want to thank my wife, Varya, for her unconditional love, care, faith and support. 


\section{VITA}

August 23, 1977.................Born, Nikolaev, Ukraine

1997...............................S., Moscow Institute of Physics and Technology, Dolgoprudnyi, Moscow Region, Russia

1997..........................Graduate Teaching Assistant, Physics Department, The Ohio State University

1998-present..................Graduate Research Assistant, Physics Department, The Ohio State University

\section{PUBLICATIONS}

\section{Research Publications}

1. Andrey I. Meshkov, Frank C. De Lucia, "Broadband absolute absorption measurements of atmospheric continua with millimeter wave cavity ringdown spectroscopy," Review of Scientific Instruments, 76, 083103 (2005)

\section{FIELDS OF STUDY}

Major Field: Physics 


\section{TABLE OF CONTENTS}

$\underline{\text { Page }}$

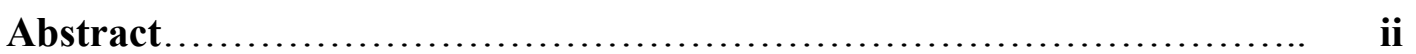

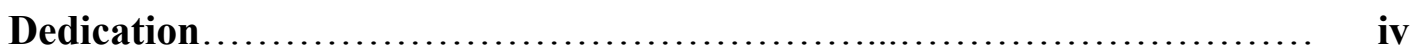

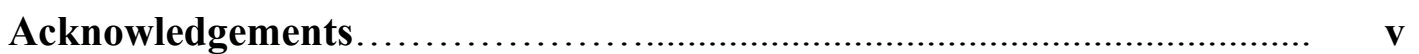

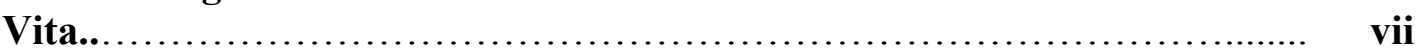

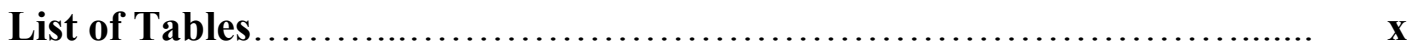

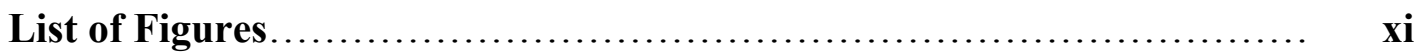

Chapters:

1. Introduction...................................................... 1

2. Microwave Atmospheric Absorption........................... 5

2.1 Radiative Transfer............................................. 5

2.2 Spectral Line Absorption................................. 8

2.3 Atmospheric Main Gaseous Absorbers...................... 11

2.3.1 Nitrogen.............................................. 11

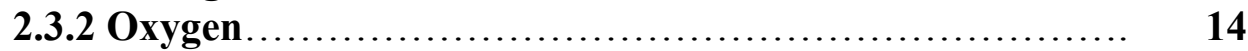

2.3.3 Water ................................................. 18

2.4 Moist Air Absorption Models................................ 19

3. Experiment.................................................. 27

3.1 Introduction.................................................. 27

3.2 Resonator Losses............................................. 29

3.2.1 Mirror Reflection Loss............................... 29

3.2.2 Diffraction Loss..................................... 30

3.2.3 Coupling Schemes and the Coupling Losses.............. 35

3.3 Frequency vs. Time Domain Measurements,

Microwave Ringdown....................................... $\quad \mathbf{4 0}$

3.3.1 Fabry-Perot Resonator Frequency Response.............. 40

3.3.2 Frequency Domain Measurements..................... 44

3.3.3 Time Domain Measurements, Microwave Ringdown...... 47

3.4 System Overview......................................... 50

3.4.1 The Basic System.................................. 50

3.4.2 Fabry-Perot Resonator............................... 51

3.4.3 Pressure and Temperature Effects..................... 56

3.5 Experimental Procedures...................................... 60 


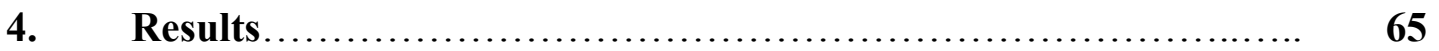

4.1 Empty Resonator Loss.................................. 65

4.2 Systematic Effects....................................... 67

4.2.1 Index of Refraction Effects, Background Pattern, Ar Measurements...................................... 68

4.2.2 Temperature Effects................................. 72

4.2.3 Moist Air Specific Effects............................ 73

4.2.4 Detection Bandwidth Effects.............................. 74

4.3 Filled Resonator Frequency Calibration,

4.4 Gas Absorption Measurements............................. 84

4.4.1 Nitrogen Absorption..................................... 84

4.4.2 Oxygen Absorption.................................. 98

4.4.3 Dry air Absorption................................... 104

4.4.4 Moist Air Absorption................................ 113

4.5 183.310 GHz Water Line Pressure Broadening and Pressure

Shift Measurements..................................... 133

4.6 Conclusions and Future Plans............................. 139

BIBLIOGRAPHY ................................................ 141 


\section{LIST OF TABLES}

Table

Page

3.1 Metal room temperature DC conductivities and the corresponding reflectivity losses at $200 \mathrm{GHz}$, calculated with Hagen-Rubens relation......

4.1 Nitrogen, oxygen, and dry air index of refraction measurement results.....

4.2 Measured nitrogen absorption coefficients $C(T) \ldots \ldots \ldots \ldots \ldots \ldots \ldots \ldots . \ldots 28$

4.3 Oxygen absorption fitting coefficients............................ 102

4.4 Dry air collision induced absorption coefficient $C(T) \ldots \ldots \ldots \ldots \ldots \ldots . \quad 108$

4.5 Moist air foreign continuum coefficient $C_{\text {Foreign }}$ temperature dependence.

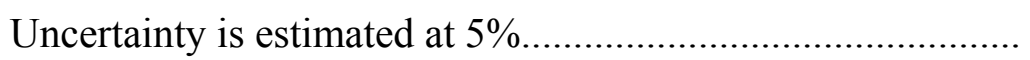

4.6 $183 \mathrm{GHz}$ water transition pressure broadening and pressure shift temperature dependence............................................... 


\section{LIST OF FIGURES}

Figure

2.1 Nitrogen absorption as a function of frequency for various temperatures from Stone et al. ${ }^{25}$

$2.2 \mathrm{O}_{2}$ spin-rotational energy levels and allowed transitions ${ }^{17}$ (transition

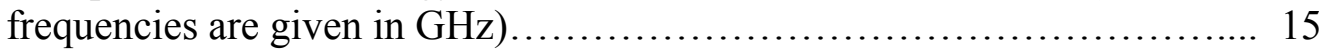

2.3 Dry air absorption below $1000 \mathrm{GHz}$ calculated with MPM89 $9^{28}$ for $24{ }^{0} \mathrm{C}$ and $101 \mathrm{kPa}$

2.4 Moist air absorption below $1000 \mathrm{GHz}$ calculated for a number of different humidities with MPM89 $9^{28}$ for $24{ }^{0} \mathrm{C}, 760$ Torr total pressure.

2.5 The calculated by $\mathrm{Ma}$ and Tipping $\mathrm{H}_{2} \mathrm{O}-\mathrm{N}_{2}$ continuum absorption (solid line) compared with MPM89 (dotted) and MPM93 (dashed) predictions, taken from $\mathrm{Ma}$ and Tipping ${ }^{9}$

3.1 A resonator, composed from two mirrors with radii of curvature $R_{1}$ and $R_{2}$, separated by the distance $L$

3.2 The mirror spot size $\omega_{m}$ and the spot size at the waist of the beam $\omega_{0}$ for a symmetric resonator with fixed mirror separation $L$ as a function of the mirror curvature $R$.

3.3 Diffraction loss $\delta_{d}$ (in \%) as a function of the mirror radius and the beam waist size.

3.4 Block diagrams of the two coupling schemes to couple the radiation in and out of the resonator cavity.

3.5 Dielectric coupling film 
3.7 Resonator response as a function of frequency. Numerical computation results for $0.1 \mathrm{GHz} / \mathrm{s}$ and $30 \mathrm{GHz} / \mathrm{s}$. Experimental results for $30 \mathrm{GHz} / \mathrm{s}$, routinely used in the FASSST measurements

3.8 Block diagram of the experimental setup

3.9 Empty resonator loss per pass as a function of frequency. Single scan, taking about 3 seconds and containing $\sim 6000$ ringdowns is shown. For the $10 \mathrm{~m}$ mirror separation, absorption of $10^{-5} \mathrm{~cm}^{-1}$ is equivalent to $1 \%$ loss per pass.

3.10 Measured resonator loss per pass comparison with the calculated losses. Calculated reflection, diffraction, input and output coupling losses are shown along with the total calculated loss and the total measured loss

3.11 Mirror reflectivity loss, calculated by subtracting the Mylar coupling in, the aperture coupling out and the diffraction losses from the measured total resonator loss per pass. Solid line is a fit of the $200-270 \mathrm{GHz}$ range to a square root frequency behavior.

3.12 Temperatures of the five chamber sections during the cool down to about $-80{ }^{0} \mathrm{C}$ and the chamber return to the room temperature after the nitrogen cooling was turned off.

3.13 The empty resonator mirrors separation as a function of temperature, calculated from the measured resonator free spectral range. Solid line is a linear fit of the data...

3.14 Triggering scheme. The sum $\mathrm{V}_{\mathrm{r}}+\mathrm{V}_{\mathrm{j}}$ of the ramp voltage and the SVC jump voltage is applied to the BWO, resulting in a rapid frequency jump of the source frequency....

3.15 Typical amplitude and mode voltage separation variations of the resonator modes. Higher order FP modes are clearly visible, especially at the high frequency end of the scan.

3.16 Absorption of 1 Torr of water vapor. The five lines used for the frequency calibration are marked.

4.1 Subtraction of two consecutive scans (grey) and two groups of 50 scans, averaged (black), of the empty resonator

4.2 Baseline structure of the empty resonator loss. 50 scans averaged, Fourier Transform processed. The structure on the zoomed part of the graph is due to the detector reflections. 
4.3 FT processed loss of the resonator filled with 2280 Torr (3 atm) of Ar, after subtracting the empty resonator loss. No averaging (grey) and 250 points average $(\mathbf{\Lambda})$

4.4 Moist continuum absorption dependence on the product of water and dry air pressures at $-23^{\circ} \mathrm{C}$. Two days of data are shown with the same temperature and close starting water pressures (at 1 atmosphere of the total moist air pressure)

4.5 Moist air absorption with 2 Torr partial water pressure. The solid line is a fit to the Van Vleck-Weisskopf profile and a quadratic in frequency continuum absorption term. Only frequencies with absorption below one half of the peak absorption ( $\square$ ) are used in the fit. The rest of the points ( $\square$ ) are presented on the same graph, showing no signs of the line profile distortion related to insufficient detecting bandwidth.

4.6 Typical small scale structure of the frequency-voltage dependence of a BWO. Resonance separation as a function of the resonance number for frequency scans of an empty resonator (black) and a resonator filled with $3 \mathrm{~atm}$ of nitrogen (red)....

4.7 The BWO small scale structure shift $d k$ of the resonator filled with 3 atm of nitrogen with respect to an empty resonator as function of the FabryPerot resonance number $k$. The residual of the fit to a linear function is presented above....

4.8 The room temperature nitrogen, oxygen, and dry air indices of refraction pressure dependence. The residual of the nitrogen data fit to a linear function is presented above.

4.9 Nitrogen, oxygen, and dry air index of refraction temperature dependence. Solid lines are fits to the power law temperature dependence.

4.10 Absorption of $3 \mathrm{~atm}$ of pure nitrogen as a function of frequency. Single scan, no averaging (grey), 66 point averages (black markers) and the $v^{2}$ fit (solid black line).

4.1150 scan-averaged absorption of 3 atm of pure nitrogen as a function of frequency. Systematic effect in the form of the standing waves of varying periods can be seen after the averaging.

4.12 The magnitude of the room temperature nitrogen absorption $v^{2}$ fit coefficient as a function of the nitrogen pressure squared. Solid line is a linear fit of the data. 
4.13 Nitrogen absorption temperature dependence. Solid line is a result of the temperature power law fit. Dotted lines are the $3 \sigma$ confidence bands. The residual of the fit is shown at the top of the figure on an expanded scale.

4.14 A subset of 100 point averaged nitrogen absorption data for a number of temperatures and pressures. Pressures are 2280 Torr (3 atm), unless otherwise noted. Solid line is a fit function given by equation (4.20)......

4.15 Histogram of the nitrogen global fit deviation.

4.16 Comparison of our measurements of the $C(T)=\frac{\alpha_{\text {Nitr }}}{P^{2} v^{2}}$ with measurements made by Ho et al. ${ }^{67}$ and Dagg et al. ${ }^{68}$

4.17 Room temperature nitrogen absorption measurements $C(300 K)=\frac{\alpha_{N i t r}(300 K)}{P^{2} v^{2}}$ by Birnbaum and Maryott ${ }^{66}$ at $24.35 \mathrm{GHz}(\mathbf{\square})$, Ho et al. ${ }^{67}$ at $9.26 \mathrm{GHz}(\bullet)$, Dagg et al. ${ }^{68}$ at $70 \mathrm{GHz}(\boldsymbol{\Delta})$, Dagg et al. ${ }^{69}$ at $140 \mathrm{GHz}(\boldsymbol{\nabla})$, Occelli et al. ${ }^{70}$ at $152 \mathrm{GHz}, 316 \mathrm{GHz}$, and $403 \mathrm{GHz}$, averaged ( $\mathbf{(})$ comparison with our measurements ( $\mathbf{4})$. All measurements are scaled to $300 \mathrm{~K}$ using a 3.4 temperature exponent.

4.18 Comparison of our room temperature 3 atm oxygen absorption measurements with the Liebe MPM89, MPM92 and MPM93 models......

4.19 Room temperature oxygen absorption measurements. Solid lines are results of the fit with parameterization given by 4.27 .

4.20 The fit coefficient $C_{1}(T)$ for the constant in frequency oxygen absorption term temperature dependence

4.21250 point average of the room temperature absorption for 3 atm of nitrogen, oxygen, and dry air. Straight lines are fits to a simple quadratic in frequency dependence for nitrogen and the dry air....

4.22 Comparison of the Debye absorption of the Liebe MPM93 model ( $\square$ ) with results of the fit of our data with equation (4.30) ( $\square$ )

4.23 Temperature dependence of the dry air collision induced absorption. Solid line is a result of the temperature power law fit. Dotted lines are the $3 \sigma$ confidence bands.

4.24 A subset of 100 point averaged dry air absorption data for a number of temperatures and pressures. Pressures are 2280 Torr (3 atm), unless otherwise noted. Solid line is a fit function given by equation (4.20). 
4.25 100 point averaged room temperature absorption of $3 \mathrm{~atm}$ of dry air $(\boldsymbol{\Delta})$. Liebe MPM89 prediction including the oxygen lines contributions (..), without oxygen lines (---), and without oxygen lines, but with the CIA contribution multiplied by 1.3 (solid line). The solid line is also the result of the fit of our data, given by 4.37 .

4.26 The 20 point averaged room temperature moist air absorption for the 760 Torr total pressure and $0.35,1.0,1.5$ and 2.4 Torr of water partial pressure $(+)$. Solid lines are predictions of the MPM89. 760 Torr dry air absorption $(\bullet)$ is shown to demonstrate the relative size of the effect.

4.27 30 point average of the moist air absorption for $1 \mathrm{~atm}$ total pressure and 1 Torr of water vapor pressure. Solid line is a fit to a Van Vleck - Weisscopf line profile and a frequency squared term. The residual of the fit is shown at the top of the figure on an expanded scale.

4.28 The moist air foreign continuum $A_{\text {Foreign }}$ vs. the product of the dry air and water pressures. The starting total pressure is 760 Torr, the starting water vapor pressure is 2.4 Torr. The residual of the fit to a straight line is shown at the top of the figure on an expanded scale.

4.29 The moist air foreign continuum coefficient $A_{\text {Foreign }}$ vs. the product of the dry air and water pressures. 760 Torr total pressure, $0.35,1.0,1.5,1.9$, and 2.4 Torr of water (red). The pump down from the starting total pressure of 760 Torr and water vapor pressure of 2.43 Torr (black). The pump down from the starting total pressure of 380 Torr and the water vapor pressure of 1.2 Torr (blue)

4.30 The $F\left(P_{D r y}\right)$ dependence on the dry air pressure for 760 Torr total and 2.4 Torr water starting pressures. Solid line is a linear fit of the data....

4.31 Pure water continuum coefficient $A_{\text {MoistCont }}$, divided by the water pressure, as a function of the water pressure

$4.321 \mathrm{~atm}$ moist air foreign continuum absorption coefficient $A_{\text {Foreign }}$ for a number of temperatures and water pressures

$4.33-43{ }^{\circ} \mathrm{C}$ moist air foreign continuum coefficient $A_{\text {Foreign }}$ vs. the product of the dry air and water pressures.

4.34 The temperature dependence of the moist air foreign continuum coefficient $C_{\text {Foreign }}$, measured at $1 \mathrm{~atm}$. Solid line is a fit to a power law. Dotted lines are the $3 \sigma$ confidence bands. 
4.35 Moist air continuum coefficient as a function of the product of the dry air and water pressures. Starting water pressure is 2.4 Torr. Starting total pressure is 760 Torr. Full moist continuum $A_{\text {MoistCont }}$ (red), foreign continuum $A_{\text {Foreign }}$ (black), calculated by subtracting the water self continuum and the dry air continuum, and the foreign continuum $A_{\text {Foreign }}^{W L}$ (blue), calculated by further subtracting the linear with water term $A_{W L}$ from the fit with equation (4.49) is shown. Respective residuals of the fit to a straight line are shown at the top of the figure on an expanded scale.

4.36 Moist air absorption continuum coefficients $C_{W L}(\boldsymbol{\Lambda}), C_{F o r e i g n}^{W L}(+)$, and $C_{\text {Foreign }}(\mathbf{-})$ for the five starting water pressures from 0.35 to 2.4 Torr. $C_{W L}$, deduced from the pure water measurements, is shown as a straight line on the top plot.

4.37 The temperature dependence of the foreign moist air continuum coefficient $C_{\text {Foreign }}^{W L}(\mathrm{red}), 1 \mathrm{~atm}$ moist air foreign continuum absorption coefficient $C_{\text {Foreign }}$ (black) and the MPM 89 prediction (green).............

4.38 The addition of the third mirror to aid in separating the gas phase absorption from the rest of the resonator losses.

4.39 Room temperature $183 \mathrm{GHz}$ water line width as a function of the total pressure for four water starting pressures from 0.35 to 2.4 Torr. Solid line is a linear fit of the data. Residuals of the fit are shown at the top of the figure on an expanded scale. ...

4.40 Room temperature $183 \mathrm{GHz}$ water line center frequency as a function of the total pressure for four water starting pressures from 0.35 to 2.4 Torr...

4.41 $183 \mathrm{GHz}$ water line pressure broadening coefficient $\gamma_{B}$ temperature dependence. Solid line is a fit to a power law. Dotted lines are the $3 \sigma$ confidence bands.

4.42 $183 \mathrm{GHz}$ water line pressure shift coefficient $\gamma_{S}$ temperature dependence. Solid line is a fit to a power law with the temperature exponent fixed at $-1.39$ 


\section{CHAPTER 1}

\section{INTRODUCTION}

We all live in a huge natural laboratory we call the atmosphere. Understanding its properties is of great importance both because it presents interesting fundamental problems and because quantitative measurements of the atmospheric properties lead directly to improvements in our everyday lives. Spectroscopy provides us with one of the best-suited tools for atmospheric studies.

Out of all spectral regions, microwaves (I will use this as a general term to include centimeter, millimeter and submillimeter waves, covering the full subterahertz frequency range) play a special role in the remote sensing of the atmosphere. Microwaves can penetrate clouds, their scattering by aerosol particles is negligible. Microwaves provide both day and night coverage since the blackbody radiance for a source at the typical atmospheric temperature peaks around $15 \mathrm{THz}$ and gives enough intensity in the microwave region. Plus, the ground state population at atmospheric temperatures is much higher than the population of vibrationally excited states, giving us the benefit of dealing with pure rotational transitions of the ground state which are easier to understand and are less subjected 
to perturbations. Pressure broadened line profiles contain a wealth of useful information and collisions are the dominant broadening mechanism in the stratosphere.

It has been known at least since the measurements near atmospheric pressure of Becker and Autler ${ }^{1}$ that all of the atmospheric absorption can not be accounted for by standard lineshape theory. Remarkably, in the intervening years, no consensus even on the physical origins of this "excess" absorption has emerged. For contributions associated with atmospheric water, suggestions have included contributions from the wings of the strong lines around $1 \mathrm{THz}$, contributions from the wings of nearby lines, dimers and polymers, etc. ${ }^{2-8}$

These diverse physical explanations result in no small part from a lack of experimental constraints, especially from laboratory experiments with well characterized molecular mixtures that have been observed over a range of frequencies, temperatures and pressures. In fact, only measurements at a few discrete frequencies, mostly at or above room temperature have been made. ${ }^{4,9}$ It has been customary to account for this extra absorption in atmospheric models by the inclusion of an empirical continuum, typically defined as whatever absorption remains after the contributions of individual lines and their lineshapes have been removed. $^{10}$

While the continuum contributions can be large, often more than half of the opacity in the atmospheric windows, ${ }^{4}$ the difficulty (both in the lab and in the atmosphere) of accurately characterizing the temperature, pressure, and especially 
the molecular content of samples has made experimental quantification difficult. Moreover, some of the contributions while weak in the laboratory have significant impact on long path measurements, especially in satellite limb sounding geometries.

For reasons that are closely related to $1 / \mathrm{f}$ noise, it is easier to measure with accuracy a change (especially if it is "rapid" in time, frequency, etc.) than it is to measure an absolute quantity. Low pressure gas phase spectroscopy takes advantage of this to separate spectral lines from slowly varying baseline caused by power fluctuations in sources, interference effects, etc. Because Doppler broadened lines are typically very narrow, $\Delta v / v \sim 10^{-6}$, this has been highly successful and very small fractional absorptions are routinely studied.

However, the quantification of slowly varying phenomena, such as spectra in solids or absorption associated with high pressure gases is much more difficult, especially if these absorptions are weak or need to be measured with high accuracy. The usual approach to this problem at long wavelength has been measurement via phase lock techniques of the change of cavity $Q$ with changes in sample concentration. ${ }^{11-15}$ However, even in these cases $1 / \mathrm{f}$ effects can be important as cavity Q changes with time due to variations in temperature, coupling, sample concentration, etc.

In this thesis, an instrument for the measurement of continua effects in the millimeter and submillimeter (a. k. a. terahertz) spectral region for mixtures of gases is developed. The instrument is based upon a fast scanning cavity ring down approach that makes possible the measurement of continua and broad line absorptions at $\sim 6000$ distinct cavity frequencies from 170 to $260 \mathrm{GHz}$ in 
$\sim 3$ seconds. The instrument's $10 \mathrm{~m}$ Fabry Perot cavity $\left(\mathrm{Q} \sim 5 \times 10^{6}\right)$ is in a chamber whose temperature can be controlled between $-80{ }^{\circ} \mathrm{C}$ and $+100{ }^{\circ} \mathrm{C}$ and whose pressure can be varied between $\sim 10^{-5}$ Torr and 10 atmospheres.

The 3 second scan results in statistical uncertainty in the absorptions of $\sim 2 \times 10^{-7} \mathrm{~cm}^{-1}$ at each of the 6000 frequencies. From these data an uncertainty of less than $1 \%$ for the weakest atmospheric continua (the dry air continuum) can be determined. For comparison, the continuum parameters that might be considered for inclusion in atmospheric remote sensing retrievals vary over a range of $10 \%$ to more than $50 \%$. This uncertainty significantly impacts quality and validity of atmospheric retrievals from a variety of remote sensing platforms. While much lower statistical uncertainties are achievable with modest additional observation times, it is much more important that the near simultaneous measurements at many frequencies produce a data set that contains, without the use of parameters from other experiments, all of the required experimental information. This approach significantly reduces systematic effects and makes possible more realistic assessment of uncertainties. 


\section{CHAPTER 2}

\section{MICROWAVE ATMOSPHERIC ABSORPTION}

\subsection{RADIATIVE TRANSFER}

Radiation interacts with the atmosphere via one of two processes: extinction (absorption and scattering) and emission. Measuring the details of these interactions by either measuring absorption of radiation from an external source or measuring emission of the atmosphere can provide us with invaluable information on atmospheric gas mixing ratios, number density and temperature profiles.

In this chapter I will give a short overview of the basic radiation transfer theory, describe the formalism for calculating microwave absorption due to the gas spectroscopic lines, and discuss in detail the microwave absorption patterns of nitrogen, oxygen and water as well as an excess continuum atmospheric absorption.

Radiation interacts with matter via one of two mechanisms: extinction (decreases radiation intensity $I_{v}$ ) and emission (increases radiation intensity). Extinction can be written in a differential form as

$$
d I_{v}(\text { extinction })=-e_{v} I_{v} d s
$$


Similar equation can be written for the emission

$$
d I_{v}(\text { emission })=S_{v} d s
$$

where $e_{v}$ and $S_{v}$ are simply proportionality coefficients called the extinction coefficient and the source term, respectively. Full radiation interaction with air can be now written as

$$
d I_{v}=d I_{v}(\text { extinction })+d I_{v}(\text { emission })
$$

or

$$
\frac{d I_{v}}{d s}=-e_{v} I_{v}+S_{v}
$$

Equation (2.4) is a differential form of the radiative transfer equation. It looks simple but determination of the $e_{v}$ and $S_{v}$ is where the difficulties are hidden.

In general, extinction coefficient for the atmosphere can be written as a sum of the air absorption, air scattering, aerosol absorption and aerosol scattering coefficients. Because the microwave wavelengths $(0.3 \mathrm{~mm}$ and larger $)$ are much larger than the air molecules, air scattering is negligible at microwave frequencies. For the non-scattering, clear atmosphere (a good approximation for the entire stratosphere and above) the only important extinction mechanism is air absorption $\alpha_{v}$.

The source term for the atmosphere in local thermal equilibrium (valid up to mesosphere for rotational states of air molecules) is given by the absorption coefficient times the Planck function $B_{v}(T)^{16}$

$$
\begin{gathered}
S_{v}=\alpha_{v} B_{v}(T), \\
B_{v}(T)=\frac{2 h v^{3}}{c^{2}} \frac{1}{e^{\frac{h v}{k T}}-1} .
\end{gathered}
$$


Now equation (2.4) can be solved analytically which leads to the integral form of the radiative transfer equation

$$
I_{v}(0)=\int_{0}^{\infty} \alpha_{v}(s) B_{v}(T(s)) e^{-\tau_{v}(s)} d s
$$

with the opacity $\tau_{v}(s)$ defined as

$$
\tau_{v}(s)=\int_{0}^{s} \alpha_{v}\left(s^{\prime}\right) d s^{\prime}
$$

It is clear now that given the atmospheric attenuation, one can uniquely calculate the radiation intensity at the detector. The inverse is not true. Different atmospheric conditions can produce the same radiation intensity at the point of measurement. Retrieving the atmosphere profiles is therefore an object of intricate frequency and altitude scan schemes as well as complicated mathematical deconvolution modeling. Not surprisingly, understanding of the frequency and temperature behavior of the atmospheric gas absorption is crucial for the atmospheric profile retrieval. 


\subsection{SPECTRAL LINE ABSORPTION}

In general, the absorption coefficient for an individual spectral line is given by

$$
\alpha(v)=n S(T) F(v)
$$

where $n$ is the number density of the absorbing molecules, $S(T)$ is the transition line intensity and $F(v)$ is the line shape function.

Line intensities of microwave rotational transitions depend on fundamental molecular quantities like the dipole moment and partition function and are readily available for all of the relevant atmospheric gases from the spectroscopic databases like the JPL database ${ }^{17}$ or HITRAN ${ }^{18}$. These databases contain $S(T)$ for some reference temperature $T_{0}$ with simple conversion to other temperatures ${ }^{17}$

$$
S(T)=S\left(T_{0}\right) \frac{T}{T_{0}} \frac{Q\left(T_{0}\right)}{Q(T)} \exp \left(\frac{E_{f}+E_{i}}{2 k T}\left(1-\frac{T}{T_{0}}\right)\right) .
$$

One only needs to know the energy levels for the transition (available in the databases) and partition functions (also available either as mathematical expressions or as a table for a set of temperatures).

This leaves us with a line shape function. There are three mechanisms involved in forming the line profile - natural line broadening due to the finite lifetime of an excited state, Doppler (or thermal) broadening due to molecular motion and collision (or pressure) broadening. Natural line broadening is completely negligible for microwave transitions in the atmosphere. Doppler broadening has a simple Gaussian profile with a half width at half maximum of ${ }^{19}$

$$
\gamma_{D}=\frac{v}{c} \sqrt{\frac{2 k T}{m} \ln 2}
$$


and should be taken into account by convoluting it with the pressure broadened line profile in the upper stratosphere and above.

All the way up to the upper stratosphere the main line shape-forming mechanism is collisional broadening. Collisional broadening is much more complicated than the Doppler broadening and its theoretical calculation formalism is ever evolving.

In fact there is no theoretical model so far that would allow derivation of a line shape function that is valid both at the line center and far wings of the line. All models have to rely on approximations that make them valid only in some regions of the line profile. The most widely used in radiative transfer modeling is the Van Vleck-Weisskopf line shape $\mathrm{e}^{20}$

$$
F_{V V W}(v)=\left(\frac{v}{v_{0}}\right)^{2} \gamma_{c}\left(\frac{1}{\left(v-v_{0}\right)^{2}+\gamma_{c}^{2}}+\frac{1}{\left(v+v_{0}\right)^{2}+\gamma_{c}^{2}}\right) .
$$

It is worth mentioning that since this line shape function is derived in the impact approximation (collision duration is short compared to the time between the collisions) it is, strictly speaking, only derived to describe the line center region and can not be fully trusted in the far wings of the line.

Unlike for the Doppler broadening, pressure broadened line width is not easily calculable and generally has to be measured in the laboratory. For atmospheric pressures a pressure broadened line width can be considered as a linear function of all of the collisional partners partial pressures. In practice though, only "self" and "air" collisions are usually considered for atmospheric studies.

$$
\gamma_{c}=C_{\text {air }}\left(P_{\text {air }}-P_{\text {self }}\right)+C_{\text {self }} P_{\text {self }} .
$$


Pressure broadening is also temperature dependent. Its functional dependence on the temperature is usually assumed to be a power law ${ }^{21}$

$$
\gamma_{c}(T)=\gamma_{c}\left(T_{0}\right)\left(\frac{T_{0}}{T}\right)^{n}
$$

Equations (2.13) and (2.14) can be combined to write the final expression for the collision broadened line width as

$$
\gamma_{c}(T)=C_{\text {air }}\left(P_{\text {air }}-P_{\text {self }}\right)\left(\frac{T_{0}}{T}\right)^{n_{-} \text {air }}+C_{\text {self }} P_{\text {self }}\left(\frac{T_{0}}{T}\right)^{n_{-} \text {self }}
$$

As was mentioned before, values of $C_{\text {air }}, C_{\text {self }}, n_{-}$air, $n_{-}$self have to be measured in the laboratory and tabulated for all the relevant transitions of all the important atmospheric gases.

It seems like we have now built a straightforward recipe for calculating the absorption of any gas with known composition and thermodynamic variables. Empirically though it has been known for a long time ${ }^{22}$ that actual absorption in the atmosphere is greater than given by the line-by-line calculations. Some insight into this discrepancy will be given next. 


\subsection{ATMOSPHERIC MAIN GASEOUS ABSORBERS}

Nitrogen ( $\left.\mathrm{N}_{2}-78.08 \%\right)$, Oxygen $\left(\mathrm{O}_{2}-20.95 \%\right)$, Argon (Ar-0.93\%) and water ( $\mathrm{H}_{2} \mathrm{O}$-variable, up to a few percent) compose $99.96 \%$ of the atmosphere by the volume. ${ }^{23}$ Argon, an atomic gas, has no transitions in the microwave and is a neutral observer only adding to the system's total pressure. Nitrogen and Oxygen, although both are diatomic gases with similar molecular masses, have quite different absorption spectra and will be discussed separately below. Water is the most important (and the most controversial) atmospheric microwave absorber because of its relative abundance and permanent electric dipole moment. Of the remaining atmospheric gases, $\mathrm{CO}_{2}$ is the most abundant at $330 \mathrm{ppm}$ (parts per million) or $0.033 \%$ with no other gas with more than $10 \mathrm{ppm} . \mathrm{CO}_{2}$, a symmetric linear molecule, has no dipole moment. Remaining gases like $O_{3}$ (up to $10 \mathrm{ppm}$ in the upper stratosphere - the so called ozone layer), $C O$ (0.01 to $1 \mathrm{ppm}$ ), $\mathrm{CH}_{4}$ (up to $1.7 \mathrm{ppm}$ ), $N_{2} \mathrm{O}$ (up to $0.3 \mathrm{ppm}$ ) and others, although very important for the atmospheric chemistry, are generally themselves the object of a retrieval and only contribute to the total absorption close to the centers of their spectral lines and at higher altitudes (lower pressures, sharper lines) because of their low number density.

\subsubsection{Nitrogen}

Nitrogen is the most abundant atmospheric gas at almost $80 \%$. As a homonuclear diatomic molecule, nitrogen has no permanent electric dipole moment and therefore has no rotational spectrum due to electric dipole transitions. However there is another mechanism that allows nitrogen to absorb microwave radiation. It is 
commonly referred to as a collision-induced absorption, or CIA. When two molecules collide, a transient dipole moment is induced in both molecules, which leads to a broad, non-resonant absorption.

The induced dipole moment magnitude is a product of the electric field vector of one molecule at the location of the other and the molecular polarizability tensor $\widetilde{\beta}$

$$
\mu_{\text {induced }}^{1}=\widetilde{\beta} \cdot E_{21}
$$

Since the lifetime of the induced dipole is basically the interaction time between the molecular pair, the line width of the induced transitions can be simply estimated $\operatorname{as}^{24}$

$$
\gamma \approx \frac{1}{t_{\text {coll }}} \approx \frac{V}{d}
$$

where $d$ is an effective distance of the interaction and $V$ is a thermal molecular velocity. For the nitrogen-nitrogen interaction $d$ is about 7 Bohr radii. This leads to $\gamma$ of the order of several terahertz for the atmospheric temperature range, which clearly makes the individual lines unresolvable.

Although intrinsically much smaller than the resonant dipole transition in intensity, CIA can be significant if the gas has high number density and no other absorption mechanisms active, both being the case for $N_{2}$. Because each collision involves two molecules (neglecting higher order multimolecular collisions), CIA is generally proportional to the pressure squared. The pure rotational CIA spectrum of $N_{2}$ peaks in the $3 \mathrm{THz}(0.1 \mathrm{~mm})$ region with microwave absorption being simply the low frequency end of this spectrum, as shown in Figure 2.1, taken from the Stone et al. $^{25}$ 
Borysow and Frommhold ${ }^{26}$ derived the nitrogen CIA absorption from the basic quantum mechanical principles. They considered quadrupol and hexadecapole interactions as well as the molecular overlap induction as the dipole inducing mechanisms and used available laboratory measurements to determine molecular parameters to be used in the fit. Their model is consistent with the data of Stone et al. and can be used to predict the absorption at frequencies and temperatures not available from the laboratory results. Recently, Boissoles et al. ${ }^{27}$ used this theoretical formalism to calculate the CIA of $\mathrm{N}_{2}-\mathrm{O}_{2}$ and $\mathrm{O}_{2}-\mathrm{O}_{2}$ mixtures.

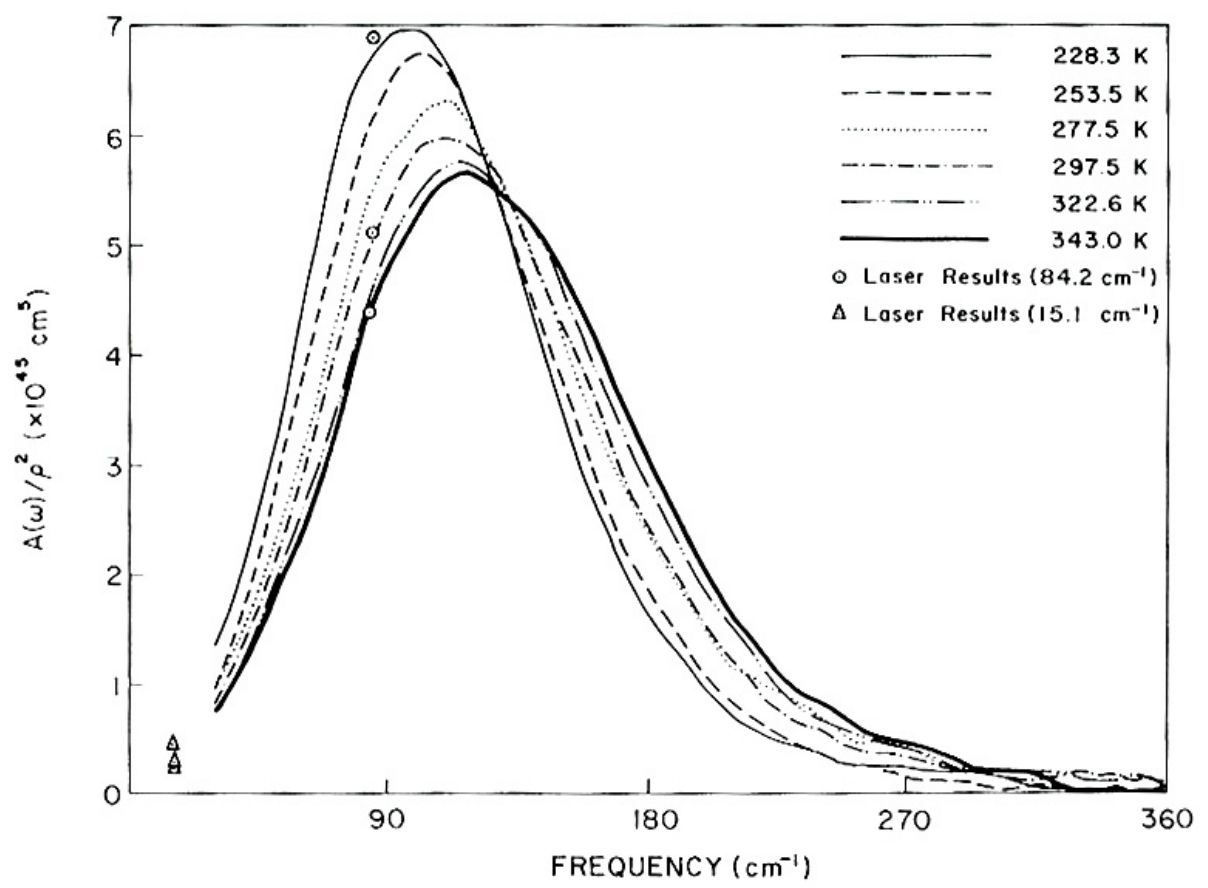

Figure 2.1: Nitrogen absorption as a function of frequency for various temperatures from Stone et al. ${ }^{25}$ 
Liebe $^{28}$ has incorporated the Stone et al. data into his atmospheric millimeterwave propagation model (MPM, to be discussed in more details later), using the following parameterization for the microwave nitrogen absorption

$$
\alpha_{N_{2}}^{M P M}=C P_{N_{2}}^{2} v^{2} \Theta^{3.5}\left(1-1.210^{-5} v^{1.5}\right),
$$

where $\Theta=\frac{300 K}{T}$. Note the pressure squared dependence as mentioned above and roughly frequency squared dependence below $300 \mathrm{GHz}$.

One last thing worth mentioning with respect to the collision-induced absorption is that $\mathrm{CO}_{2}$ has almost 75 times greater microwave CIA coefficient than nitrogen; ${ }^{29}$ yet $\mathrm{CO}_{2}$ atmospheric absorption is negligible because of the low abundance of $\mathrm{CO}_{2}$ compared to $\mathrm{N}_{2}$.

\subsubsection{Oxygen}

Just like nitrogen, oxygen is a homonuclear diatomic molecule and has no permanent electric dipole moment. The oxygen molecule, however, is unusual because although it has an even number of electrons, electron spins are not all paired. In fact, oxygen ground state is ${ }^{3} \Sigma_{1}$ with zero average orbital angular momentum along the molecular axes but with two parallel electron spins. ${ }^{19}$ Consequently the oxygen molecule has a permanent magnetic dipole. A magnetic dipole interaction is not as strong as a typical molecular electric dipole interaction (one Bohr magneton is only about $1 / 100$ of a Debye - unit of molecular electric dipole moment). Yet this intrinsic weakness of the magnetic interaction is compensated by the great abundance $(\sim 21 \%)$ of oxygen in the atmosphere. 
Coupling of the different angular momenta in the oxygen molecule is best described by the Hund's case $b$. The spin $S$ is coupled with the molecular rotation angular momentum $\boldsymbol{N}$ to give the total angular momentum $\boldsymbol{J}=\boldsymbol{N}+\boldsymbol{S}$. Spin-rotational energy levels of oxygen are shown in Figure 2.2.

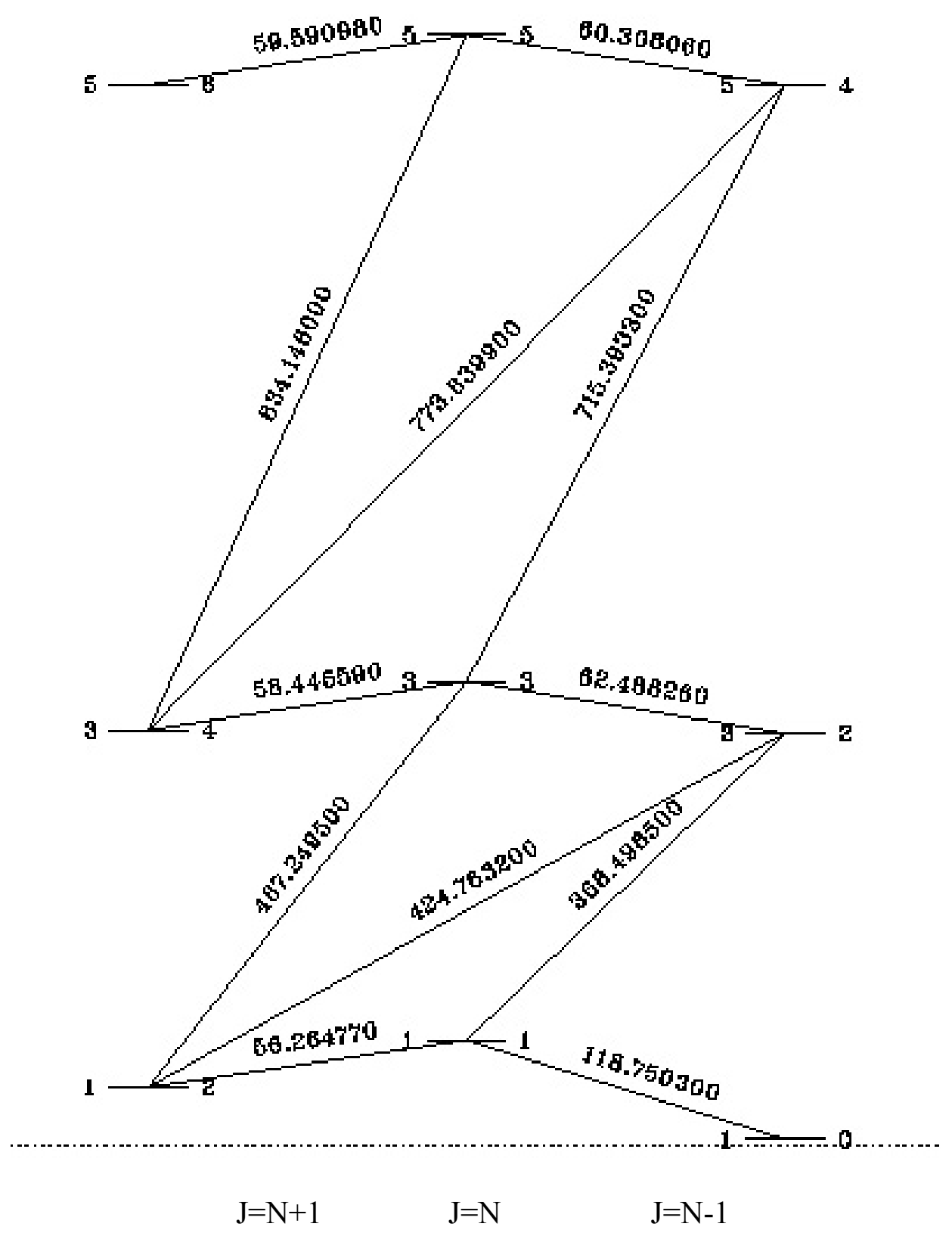

Figure 2.2: $\mathrm{O}_{2}$ spin-rotational energy levels and allowed transitions ${ }^{17}$ (transition frequencies are given in GHz). 
Magnetic dipole transitions between these levels give rise to seven single spectral lines under $1 \mathrm{THz}$ plus a group of spectral lines between $50 \mathrm{GHz}$ and $70 \mathrm{GHz}$, corresponding to transitions without the change in the molecular rotation angular momentum $N$. For $300 \mathrm{~K}, 30$ of these "60 GHz band" lines have intensities greater than $1 \%$ of the strongest $(N=9, v=61.2 \mathrm{GHz})$ line (based on the data from JPL line catalogue).

The standard formalism that was described above can be applied to calculate the absorption of all the lines above $100 \mathrm{GHz}$. One has to be more careful with the $60 \mathrm{GHz}$ band, especially in the troposphere where pressure broadened line width is much greater than the line separation. The Van Vleck - Weisskopf line shape has to be modified ${ }^{30}$ to describe the line overlap effects.

Another important oxygen absorption component is the Debye absorption. Quantum-mechanically the origin of this absorption lies in the fact that magnetic dipole matrix has diagonal elements, corresponding to non-resonant $\Delta N=\Delta J=0$ transitions. ${ }^{31}$ It can also be understood classically by assuming that during each molecular collision the radiation electric field loses energy by aligning molecular dipoles according to the Boltzman distribution $\exp \left(-\frac{E \mu}{k T}\right) \cdot 32$ Classical derivation gives the same result as quantum theory, with the (non resonant) absorption given by

$$
\alpha_{O_{2}}^{\text {Debye }}=\frac{8 \pi^{2} n \mu^{2}}{3 k T c} \frac{v^{2} \gamma}{v^{2}+\gamma^{2}}
$$

Note that Van Vleck-Weisskopf line shape reduces to the Debye case when the line center frequency $v_{0}$ is taken to be zero. 
As always, the details of $\gamma$ behavior need to be known to calculate the Debye absorption. Liebe uses the following parameterization in his MPM89 ${ }^{28}$ model

$$
\gamma_{\text {Debye }}=5.610^{-3}(p+1.1 e) \Theta(\mathrm{GHz})
$$

where $\mathrm{p}$ and e are partial pressures for dry air and water vapor in $\mathrm{kPa}$. Even for the ground pressures of $100 \mathrm{kPa}, \gamma_{\text {Debye }}$ is only about $0.6 \mathrm{GHz}$, which means that the Debye absorption is proportional to the value of $\gamma_{\text {Debye }}$ and can be considered constant for all frequencies above $10 \mathrm{GHz}$.

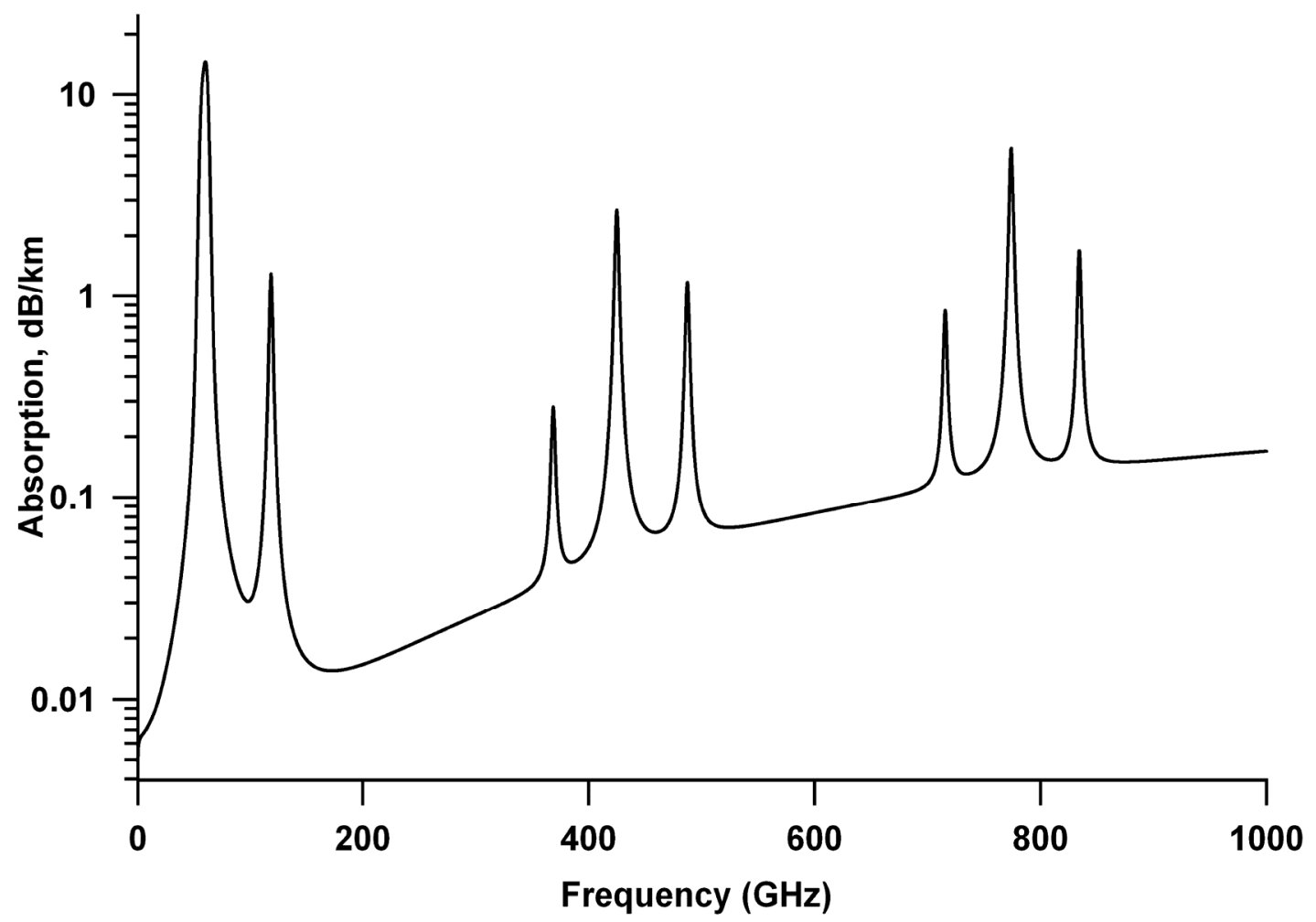

Figure 2.3: Dry air absorption below $1000 \mathrm{GHz}$ calculated with MPM89 ${ }^{28}$ for $24{ }^{\circ} \mathrm{C}$ and $101 \mathrm{kPa}$. 
To summarize, the dry air (no water vapor) atmospheric absorption in the microwave region consists of seven sharp resonant absorption lines of oxygen, a nonresonant "continuum" absorption due to the nitrogen and oxygen collision induced absorptions and a frequency independent above $10 \mathrm{GHz}$, constant oxygen Debye absorption. Prediction of the Liebe model is shown in Figure 2.3. Laboratory measurements are hard in the windows between the oxygen lines because of the small absolute value of the absorption. At $300 \mathrm{GHz}$, for example, the dry air absorption is only about $0.6 \%$ per kilometer, too small to be measured in the laboratory by a direct absorption, yet clearly big enough to be very important for atmospheric studies, where long path lengths are always encountered.

\subsubsection{Water}

The last, but most certainly not the least, water is the main microwave absorber in the atmosphere. Water number density varies greatly with temperature and altitude, going from as low as $0.1 \mathrm{ppm}$ up to $4 \%$ at the ground level. ${ }^{33}$ Yet the water contribution to the atmospheric absorption is far greater than it would be suggested by a simple abundance argument because of its small moments of inertia and its permanent electric dipole moment. Called by Liebe ${ }^{34}$ a "Nemesis for the millimeter wave propagation", water vapor plays an enormously important role in atmospheric absorption modeling.

Moreover, the explanation of the experimentally measured excess absorption far from the line centers compared to the calculated due to the separate water line absorptions has been a controversial subject of discussions for a long time now. Some of the hypotheses put forward to explain this extra absorption are far wings of the 
strong infrared lines, collision induced absorption for transitions that would not be allowed in the isolated molecule and absorption due to the water dimers.

In the next section I will give an overview of the most widely used models for calculating the moist air atmospheric absorption, discuss their advantages and limitations, describe the existing theoretical models for the excess continuum absorption and provide a summary for the current state of the water vapor attenuation in the atmosphere studies.

\section{$2.4 \quad$ MOIST AIR ABSORPTION MODELS}

Water is an asymmetric top molecule with a 1.94 Debye permanent electric dipole moment. ${ }^{19}$ It has 12 lines in the sub-terahertz region that are more than two orders of magnitude stronger than the strongest oxygen line. Three lines $(557 \mathrm{GHz}$, $752 \mathrm{GHz}$ and $988 \mathrm{GHz}$ ) are more than 5 orders of magnitude stronger. For example, close to the center of the $557 \mathrm{GHz}$ line and at high relative humidity, water vapor absorbs $99 \%$ of the radiation in just one meter. Far wings of these strong lines alone can be enough to dominate the dry air absorption. Even in the "windows" away from the line centers moist air absorption can reach up to $10 \mathrm{~dB} / \mathrm{km}$ for high frequencies even on a relatively dry day at $10 \% \mathrm{RH}$.

Any moist air absorption model needs to deal with water lines first. The line intensities are known with a great precision and are readily available in the spectroscopic databases. Line shape functions, on the other hand, both in their mathematical representation and physical behavior of the broadening parameters, differ from one model to another. 
The Millimeter-Wave Propagation Model (MPM) ${ }^{28,35-38}$ was developed by Hans J. Liebe to give a quick, computationally simple way of calculating the atmospheric absorption for different humidities at the frequency range below $300 \mathrm{GHz}$. It was later extended up to $1000 \mathrm{GHz}$ by including more lines and improving the set of continuum parameters in 1989 (MPM89) ${ }^{28}$ and 1992 (MPM92) ${ }^{38}$ and was revisited once more in 1993 (MPM93). ${ }^{37}$ Although all parameterizations remained the same, the values of MPM parameters are slightly different in different model versions. Later experimental evidence was found to be somewhere between the MPM89 and MPM93 versions, depending on the particular temperature and frequency regions considered. ${ }^{39}$

For his line-by-line calculations Liebe used six different parameters for each water line. Two parameters were used to describe the line strength and its temperature behavior and three more were needed for the pressure broadened line width characterization. Liebe provided all six parameters for each line based on either available experimental data or theoretical predictions. A standard Van VleckWeisskopf line shape function was used for the line profile calculations.

The water continuum, which in Liebe's case is simply the difference between the total absorption and the line-by-line absorption, is parameterized in the MPM as

$$
\alpha_{c}=v^{2} e\left(b_{s} e \Theta^{10.5}+b_{f} p \Theta^{3}\right)
$$

where $e$ and $p$ are again water and dry air partial pressures. The first and the second terms in this continuum parameterization are commonly referred to as the selfcontinuum and the foreign-continuum. In MPM89, $b_{s}$ is 31.5 times greater than $b_{f}$. The temperature dependence of the self-continuum is also much higher than for the foreign-continuum. Another general feature of the water continuum absorption is that this absorption increases as a frequency squared in the microwave region. 
Figure 2.4 shows the total moist air absorption, calculated for a number of different humidity values according to the Liebe MPM89 model.

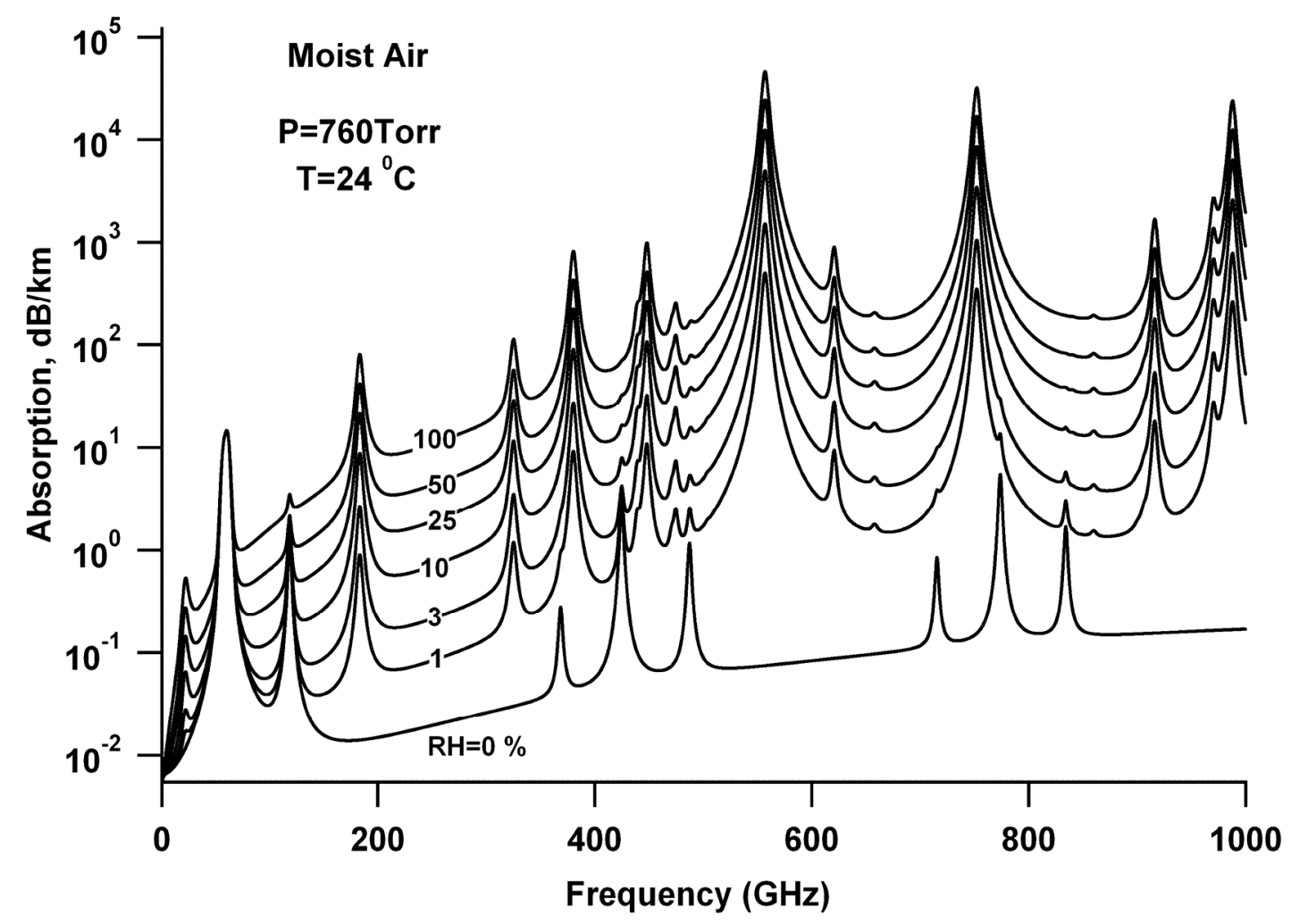

Figure 2.4: Moist air absorption below $1000 \mathrm{GHz}$ calculated for a number of different humidities with MPM89 $9^{28}$ for $24{ }^{0} \mathrm{C}, 760$ Torr total pressure.

In his model, published in 1998, Rosenkranz ${ }^{39}$ uses slightly different line shape function and incorporates experimental results that were not available in 1993, but essentially gives the same parameterization for the continuum absorption as Liebe in his MPM. Among the changes are the increase of the $b_{f}$ by $15 \%$ (partially because of the difference in the used line-shapes) and a 7.5 instead of 10.5 power law coefficient in the self-continuum temperature dependence. 
Although Liebe and Rosenkranz models succeed in giving a simple way of calculating the total atmospheric absorption at a given frequency for most of the standard atmospheric conditions, they do not even attempt to define the origin of the excess water continuum, giving instead the best fit parameterization of the experimentally measured absorption. This clearly limits their applications and makes the extrapolation to the regions where experimental data is not available questionable, with no real way to estimate how much error would be introduced. For example, most water vapor laboratory measurements so far were limited for various reasons to temperatures above the water freezing point, $273 \mathrm{~K}$. Since tropospheric temperatures go as low as $200 \mathrm{~K}$, both experimental measurements and a sound understanding of the underlying physics are required to predict the tropospheric absorption.

As mentioned above, the infrared line wings, collision induced absorption and water dimers were used as possible explanations of the water continuum. Each theory needs to be examined closely since all can, in principle, be accounted for the extra absorption.

Microwave transitions of the water dimer $\left(\mathrm{H}_{2} \mathrm{O}\right)_{2}$ have been observed in molecular beam experiments ${ }^{40}$ and a theoretical calculations based on the interaction potential were published that predict the equilibrium constants for water dimerization in the atmosphere. ${ }^{41,42}$ Water dimers relation to the atmospheric water continuum has been historically the subject of heated discussions and controversial statements. ${ }^{43}, 44$ Dimers could be a part of the self-continuum absorption, which is proportional, just as dimer concentration should be, to the water partial pressure squared. However, if dimers do have a broad absorption spectrum varying as frequency squared, the frequency dependence would seem to be accidental. ${ }^{29}$ 
Far wing theory and collision induced absorption theory are actually interrelated and both use similar mathematical models in getting their results. In a sense no absorption is possible without the interaction (collisions) with other molecules since without collisions any excited molecule would simply reemit the absorbed photon due to the finite lifetime of the excited state without any change to the molecule's kinetic energy. The term CIA is usually used to describe the absorption due to the fact that some transitions that would not be allowed in a non perturbed molecule can become allowed when the intermolecular interaction potential introduces sufficiently large perturbations to the molecular Hamiltonian. Far wing theories usually extend the standard impact approximation to include the long-range molecular interactions between the molecules. Generally speaking, both mechanisms are present for any gas; the question is only, which one is more dominant. Water collisions with nitrogen can lead to water transitions taking place that were not allowed by the selection rules for a free water molecule. This effect has not yet been rigorously calculated for any asymmetric rotor type molecule. $^{29}$

It seems though that the far wing formalism originally developed for the infrared water continuum absorption by Clough et al. ${ }^{45}$ and later improved by Ma and Tipping 2 , 9, 46-49 can be extended into the microwave region with theoretical calculations consistent with presently available experimental data.

Although the full mathematical formalism is rather complicated, the idea of the far wing absorption calculations is straightforward. ${ }^{9,}{ }^{50}$ For a low-pressure case where binary collision approximation is valid, the absorption coefficient of a gas sample per unit volume can be written as

$$
\alpha(\omega)=n_{\text {pair }} \frac{4 \pi^{2}}{3 \hbar c} \omega\left(e^{\frac{\hbar \omega}{k T}}-1\right) F(\omega)
$$


where $n_{\text {pair }}$ is a number density of $\mathrm{H}_{2} \mathrm{O}-\mathrm{N}_{2}$ collision pairs, which is proportional to the product of pressures of $\mathrm{H}_{2} \mathrm{O}$ and $\mathrm{N}_{2} . F(\omega)$ is the Fourier transform of the correlation function $C(t)$ of the dipole moment operator

$$
F(\omega)=\frac{1}{\pi} \operatorname{Re} \int_{0}^{\infty} \exp (i \omega t) C(t) d t
$$

and

$$
C(t)=\operatorname{Tr}\left(\mu^{+} \mathrm{e}^{-i H t} \rho \mu \mathrm{e}^{i H t}\right)
$$

where $\mu$ is the water dipole moment operator and $\rho$ is the pair density matrix. The total Hamiltonian for the pair can be written as the sum of the unperturbed water molecule Hamiltonian, unperturbed nitrogen molecule Hamiltonian and an interaction between the two molecules

$$
H_{\text {total }}=H_{\mathrm{H}_{2} \mathrm{O}}+\mathrm{H}_{\mathrm{N}_{2}}+H_{\mathrm{H}_{2} \mathrm{O}-\mathrm{N}_{2}}
$$

Now, given the molecular interaction potential and after making physically reasonable approximations, it is possible to numerically calculate the absorption as a function of frequency and temperature.

Ma and Tipping only included the water dipole - nitrogen quadrupole interaction for the anisotropic part of the potential and used the standard LennardJones potential as the isotropic part of the interaction Hamiltonian. Their model predictions, calculated for $270 \mathrm{~K}$ and $330 \mathrm{~K}$, are shown in Figure 2.5 along with Liebe MPM89 and MPM93 model predictions. 
The authors also provided a simple analytical parameterization of their results calculated for twelve different temperatures from $220 \mathrm{~K}$ to $330 \mathrm{~K}$, applicable for frequencies up to $450 \mathrm{GHz}$ as follows

$$
\alpha(v, T)=1.591510^{-7} P_{\mathrm{H}_{2} \mathrm{O}} P_{\mathrm{N}_{2}}\left(\frac{300}{T}\right)^{4.982} v^{2.059} \mathrm{~dB} / \mathrm{km} \quad(P \text { in } \mathrm{kPa})
$$

No uncertainties are given or discussed, but it is clear that these calculations are consistent with empirically fitted functional behavior of the foreign continuum of the atmospheric water absorption. This is the first theoretical model that is able to predict water vapor atmospheric absorption without having to add an empirical or semi empirical continuum absorption term, which is a big step forward. Yet it is too soon to close the foreign water vapor continuum term discussion. The authors, for instance, suggest that several refinements could be made to the presented calculations but that more experimental data is needed first to check the validity of their calculated results, especially at low temperatures.
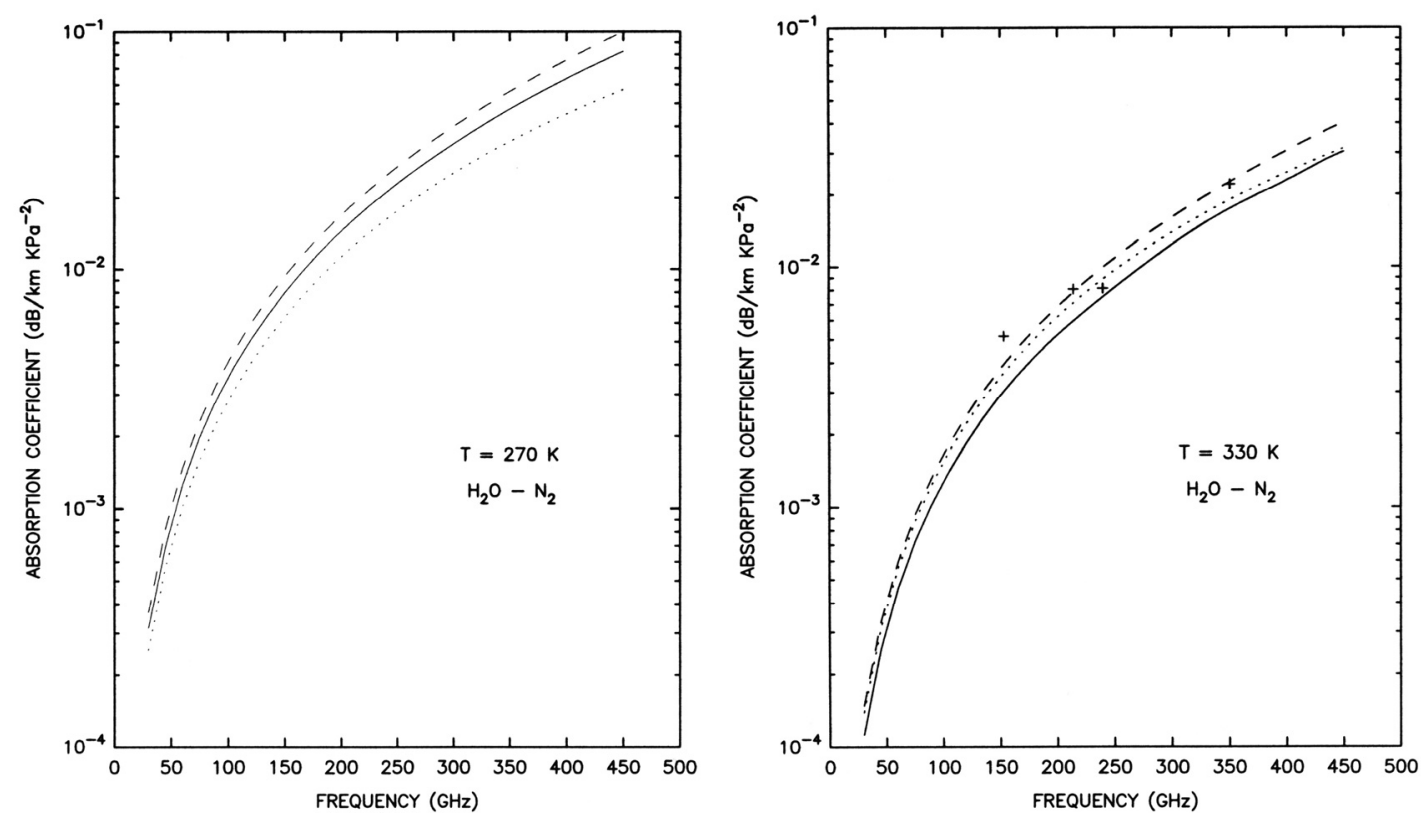

Figure 2.5: The calculated by $\mathrm{Ma}$ and Tipping $\mathrm{H}_{2} \mathrm{O}-\mathrm{N}_{2}$ continuum absorption (solid line) compared with MPM89 (dotted) and MPM93 (dashed) predictions, taken from Ma and Tipping. ${ }^{9}$ 
As for the self-broadening part of the water vapor absorption, the part that is proportional to the water partial pressure squared, the question of its physical origin is still open. Any of the described above physical mechanisms can be accountable for this term with exceptionally high temperature dependence.

Of all the atmospheric gases water vapor is the leading and yet the least understood absorber in the microwaves. In 1947 Van Vleck published a paper ${ }^{22}$ in Physical Review where he demonstrated that the theoretically calculated "residual term", or the attenuation due to far wings of the lines other than the one studied (at 22.2 GHz) is much smaller than what was measured experimentally. Van Vleck mentioned that the explanation could be that the theory of the far wing line shapes is incorrect and stated that "more study of the question, both theoretically and experimentally, is desirable".

Fifty-seven years, many scientists' man-hours and much money spent later, the same general statement can still be repeated without being too far off. Especially because the water vapor continuum absorption modeling accuracy remains one of the main limiting factors in most of the current microwave atmospheric retrieval experiments. 


\section{CHAPTER 3}

\section{EXPERIMENT}

\subsection{INTRODUCTION}

In this section I will address the fundamental physics behind the experiment operation and describe the experimental setup and the measurement procedure.

The measurement of changes in the cavity $Q$ as opposed to observations of direct signal loss due to the gas absorption has proven to be more advantageous for the measurement of wide, slowly varying with frequency, phenomena. It has been demonstrated $^{51}$ that absorption measurement sensitivities of $\sim 4 \times 10^{-9} \mathrm{~cm}^{-1}$ can be achieved with a Fabry-Perot resonator and a phase-locked radiation source. If measurements of controlled gas samples could be made with this accuracy for all the relative atmospheric conditions, this would improve the current atmospheric absorption uncertainties by orders of magnitude and provide invaluable data for the theoretical community to better understand underlying physics of atmospheric absorption. With this in mind we have developed the system described in detail in this thesis. 
Two mirror Fabry-Perot resonators have been successfully used in gas absorption measurements. The gas absorption contribution to the total loss can be extracted by either studying the $\mathrm{Q}$ behavior as a function of gas density or by subtracting the measured or calculated losses of an empty resonator.

As the radiation passes between the Fabry-Perot mirrors, its intensity decreases due to the resonator losses. The total fractional radiation intensity loss per pass $\delta_{t}$ can be written as a sum of four terms

$$
\delta_{t}=\delta_{r}+\delta_{d}+\delta_{c}+\delta_{g}
$$

where $\delta_{r}$ is the reflection loss, $\delta_{d}$ is the diffraction loss, $\delta_{c}$ is the coupling loss, and $\delta_{g}$ is the gas absorption loss. Empty cavity losses can be estimated from known parameters (e.g. reflection loss) and by use of the Gaussian beam propagation theory (e.g. diffraction loss). Mirror reflection loss depends on the choice of mirror material and is the only loss that is fundamental and unavoidable. Diffraction losses can be made as small as desired by increasing the mirror diameters. Coupling losses depend on how the radiation is coupled in and out of the resonator cavity. Because the magnitude and frequency behavior of all the resonator losses is important for the system operation, I will discuss each loss separately. 


\subsection{RESONATOR LOSSES}

\subsubsection{Mirror reflection loss}

To estimate the reflection loss of a metallic mirror in the microwave we can use the Hagen-Rubens relation

$$
\delta_{r}=2 \sqrt{\frac{v}{\delta_{D C}}}
$$

where $\delta_{r}$ is the reflection loss, $v$ is the radiation frequency and $\delta_{D C}$ is the metal direct current conductivity. Although this relation underestimates the reflection loss, especially at higher frequencies ${ }^{52}$, it can still be used as a starting point. Table 3.1 lists the room temperature DC conductivities and the corresponding reflectivity losses at $200 \mathrm{GHz}$ for copper, gold, aluminum and silver, calculated with equation (3.2). Copper was chosen as a material for the Fabry-Perot resonator mirrors because of the price-reflectivity consideration. Experimental measurements of the microwave copper conductivity found in the literature vary around $0.2 \%$ reflectivity loss when scaled to $200 \mathrm{GHz}^{52,53}$

\begin{tabular}{ccc}
\hline Metal & Conductivity $\left(10^{17} \mathrm{~s}^{-1}\right)$ & $\delta_{r}(\%)$ \\
Copper & 5.364 & 0.122 \\
Silver & 5.671 & 0.118 \\
Gold & 4.065 & 0.140 \\
Aluminum & 3.396 & 0.154 \\
\hline
\end{tabular}

Table 3.1: Metal room temperature DC conductivities and the corresponding reflectivity losses at $200 \mathrm{GHz}$, calculated with Hagen-Rubens relation. 


\subsubsection{Diffraction loss}

Well developed Gaussian beam propagation formalism can be used to calculate the supported modes and their characteristics in an optical system. Detailed derivation can be found in many textbooks, ${ }^{54,55}$ I will only present and discuss the results for the fundamental mode of the two-mirror optical resonator, that is the resonator, composed from two mirrors with radii of curvature $R_{1}$ and $R_{2}$, separated by the distance $L$, as shown in Figure 3.1.

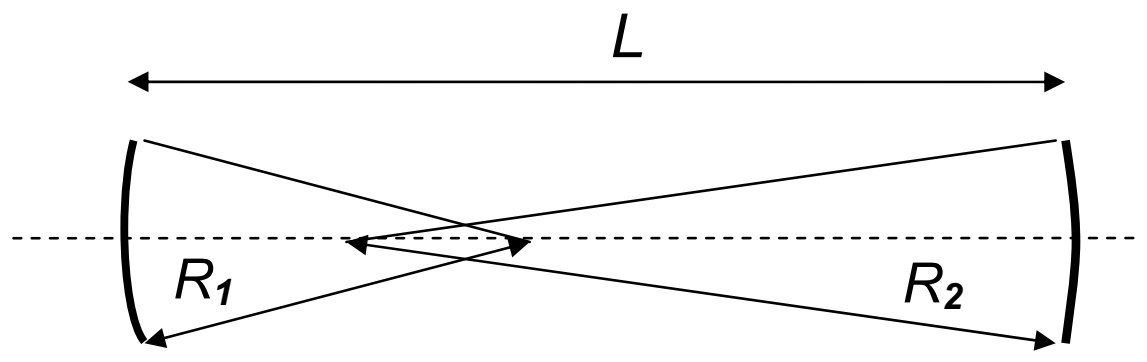

Figure 3.1: A resonator, composed from two mirrors with radii of curvature $R_{1}$ and $R_{2}$, separated by the distance $\mathrm{L}$

For this system the spot sizes (the radius where the radiation field is $e$ times smaller than in the mode center) of the fundamental mode (from now on unless otherwise noted I will only discuss the fundamental $\mathrm{TEM}_{00}$ mode) on the mirrors are

$$
\omega_{1}=\sqrt{\frac{L \lambda}{\pi} \sqrt{\frac{R_{1}^{2}\left(L-R_{2}\right)}{L\left(L-R_{1}\right)\left(R_{1}+R_{2}-L\right)}}}
$$

and

$$
\omega_{2}=\sqrt{\frac{L \lambda}{\pi} \sqrt{\frac{R_{2}^{2}\left(L-R_{1}\right)}{L\left(L-R_{2}\right)\left(R_{1}+R_{2}-L\right)}}} .
$$


The beam waist spot size is

$$
\omega_{0}=\sqrt{\frac{\lambda}{\pi} \sqrt{\frac{L\left(L-R_{1}\right)\left(L-R_{2}\right)\left(R_{1}+R_{2}-L\right)}{\left(R_{1}+R_{2}-2 L\right)^{2}}}},
$$

where $\lambda$ is the radiation wavelength.

For the symmetric case $\left(R_{1}=R_{2}=R\right)$ these formulas reduce to

$$
\omega_{1}=\omega_{2}=\sqrt{\frac{L \lambda}{\pi} \sqrt{\frac{R^{2}}{L(2 R-L)}}}
$$

and

$$
\omega_{0}=\sqrt{\frac{L \lambda}{2 \pi} \sqrt{\frac{2 R-L}{L}}} .
$$

For a resonator to be stable the following relation must be satisfied

$$
0<\left(1-\frac{L}{R_{1}}\right)\left(1-\frac{L}{R_{2}}\right)<1
$$

which simplifies for the symmetric resonator to

$$
L<2 R
$$

The size of the mirrors is what dictates the diameter of the vacuum chamber. Therefore, given the mirror separation L, the configuration with the smallest mirror mode size should be used. It can be easily checked that for the stable symmetric resonator the mirrors mode sizes are smallest if $\mathrm{L}=\mathrm{R}$. Because the focal distance of a mirror with the curvature $\mathrm{R}$ is equal to $\mathrm{R} / 2$, foci of the two mirrors coincide and this case is called confocal. 


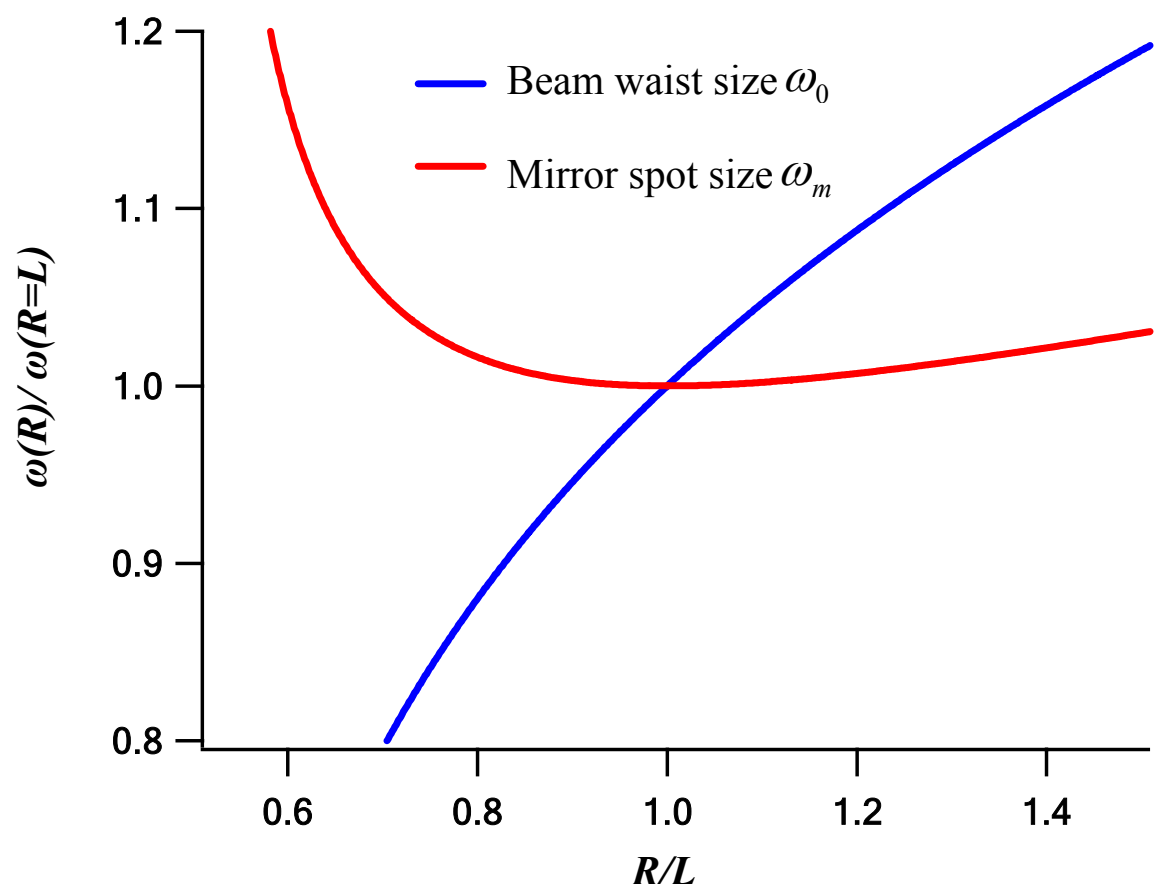

Figure 3.2: The mirror spot size $\omega_{m}$ and the spot size at the waist of the beam $\omega_{0}$ for a symmetric resonator with fixed mirror separation $L$ as a function of the mirror curvature $R$.

In practice, if the two mirrors are not exactly identical, using the confocal configuration can lead to highly uneven mode sizes on the mirrors. It can be noticed from the fact that the ratio $\frac{\omega_{1}}{\omega_{2}}=\sqrt{\frac{L-R_{2}}{L-R_{1}}}$ is a rapidly changing function when both $R_{1}$ and $R_{2}$ are close to $L$. For a symmetric resonator with fixed $L$, the mirror spot size and the spot size at the waist of the beam is plotted in Figure 3.2 as a function of the mirror curvature. If mirror curvatures are $R_{1}=R_{2} \sim 0.8 L$, it increases the mirror spot size by only about $1.6 \%$, but solves the mentioned above problem with the confocal configuration. It also reduces the beam waist size by $\sim 12 \%$. 
The radiation intensity $I(r)$ in the Gaussian beam with a spot size $\omega$ varies with the distance $r$ from the beam center as

$$
\frac{I(r)}{I(0)}=\operatorname{Exp}\left[-2\left(\frac{r}{\omega}\right)^{2}\right]
$$

From this, the diffraction loss $\delta_{d}$ can be calculated as the fraction of the Gaussian beam power passing outside of the circle of the radius $R$ :

$$
\delta_{d}=\frac{\int_{R}^{+\infty} I(r) 2 \pi r d r}{\int_{0}^{+\infty} I(r) 2 \pi r d r}=\operatorname{Exp}\left[-2\left(\frac{R}{\omega}\right)^{2}\right]
$$

the same mathematical expression as for the radiation intensity.

Plot of $\delta_{d}$ as a function of the ratio $\frac{R}{\omega}$ is shown in Figure 3.3.

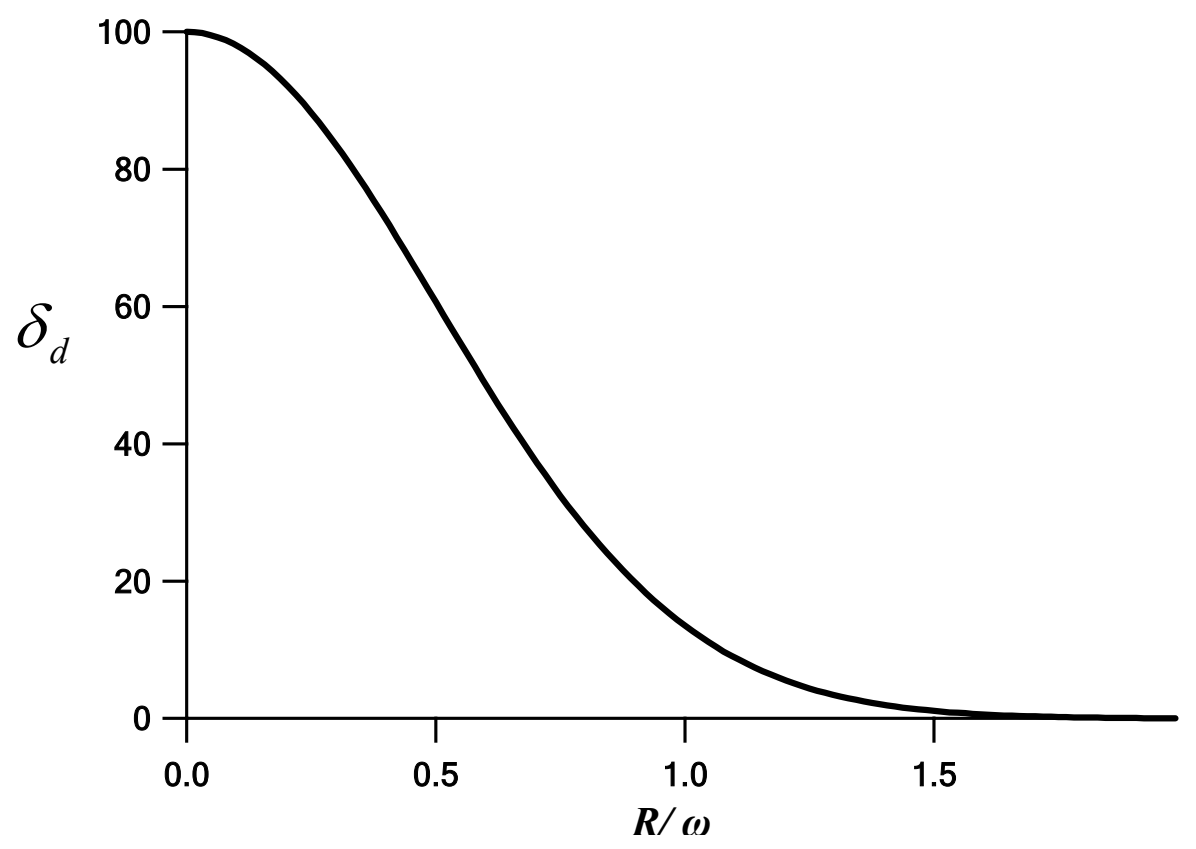

Figure 3.3: Diffraction loss $\delta_{d}($ in $\%$ ) as a function of the mirror radius and the beam waist size. 
Equation (3.11) gives the following values for the diffraction loss of the beam with a spot size $\omega$ on the mirror of radius $R: 13.5 \%$ of the total power is lost when $R=\omega$, about $1.1 \%$ is lost when $R=1.5 \omega$, and less than $0.04 \%$ is lost when $R=2 \omega$.

By substituting (3.6) into (3.11) we can derive that for the given experimental setup (fixed $R$ and $L$ ) the diffraction loss decreases exponentially with the increase in frequency as

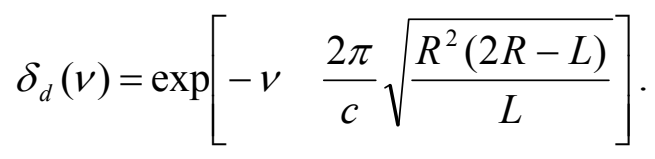




\subsubsection{Coupling schemes and the coupling losses}

In general, radiation can be coupled in and out of the resonator cavity via an aperture in one on the mirrors (hole coupling) or with a beam splitter, positioned inside the cavity. If the small (low loss) aperture is used to couple the radiation in, it radiates energy into a large solid angle, therefore decreasing the coupling efficiency. The beam splitter, used to couple the radiation into the cavity, does not have this problem and was therefore used in our experiment.

To decide on the strategy to couple the radiation out of the cavity, we have carefully considered two possible setups, schematically shown in Figure 3.4.

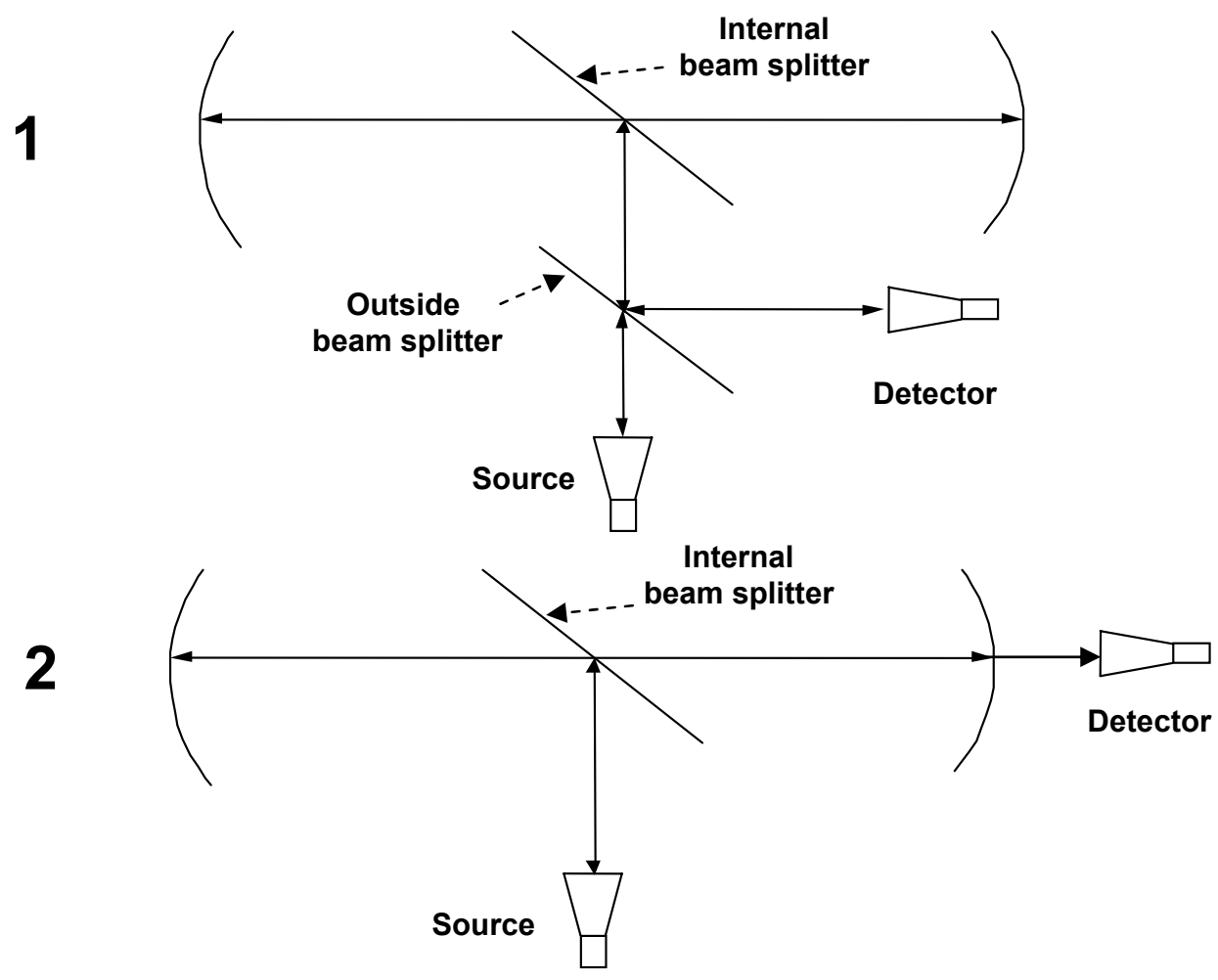

Figure 3.4: Block diagrams of the two coupling schemes to couple the radiation in and out of the resonator cavity. 
Scheme 1 uses the beam splitter to couple radiation both in and out of the cavity while Scheme 2 couples radiation in via the beam splitter and out via the aperture in one of the Fabry-Perot mirrors. To maximize the detector signal, the external beam splitter in the first setup has to reflect exactly half of the radiation. Theoretical calculations of the empty resonator loss and output intensity at the resonance are similar for both cases and lead to the following results (detailed derivation for the second scheme is given in the next section) for small coupling coefficients:

Scheme 1:

$$
\begin{gathered}
I_{1}^{\text {res }} \text { out }=I_{\text {in }} \frac{\delta_{c i}{ }^{2}}{4\left(\delta_{c i}+\delta_{o}\right)^{2}} \\
\delta_{1 t}=\delta_{o}+\delta_{c i}
\end{gathered}
$$

Scheme 2:

$$
\begin{gathered}
I_{2}^{r e s}{ }_{\text {out }}=I_{\text {in }} \frac{\delta_{c i} \delta_{c o}}{\left(\delta_{c i}+\delta_{o}+\frac{1}{2} \delta_{c o}\right)^{2}} \\
\delta_{2 t}=\delta_{o}+\delta_{c i}+\frac{1}{2} \delta_{c o}
\end{gathered}
$$

Where $I_{\text {in }}$ is the input radiation intensity, $I_{1}^{\text {res }}$ out and $I_{2}^{\text {res }}$ out are the output radiation intensities, $\delta_{1 t}$ and $\delta_{2 t}$ are the total fractional radiation intensity losses per pass for schemes 1 and 2, respectively; $\delta_{c i}$ is the internal beam splitter coupling coefficient, $\delta_{c o}$ is the coupling loss of the iris on the second scheme's coupling mirror, and $\delta_{o}$ is all other remaining system loses including the diffraction loss and the gas absorption loss, presumed to be the same for both setups. 
Let us compare the output intensities while keeping the total loss the same for both schemes. First, it is straightforward to show for the second setup that if $\delta_{2 t}$ is kept constant $\left(\delta_{c i}+\frac{1}{2} \delta_{c o}\right.$ is kept constant), than the highest output intensity is, not surprisingly, achieved when $\delta_{c i}=\frac{1}{2} \delta_{c o}$. For this case if the total coupling loss per pass is the same for both schemes, that is $\delta_{c}=\delta_{1 c i}=\delta_{2 c i}+\frac{1}{2} \delta_{2 c o}=2 \delta_{2 c i}=\delta_{2 c o}$, the output intensities for the two cases are

$$
I_{2}^{\text {res }}{ }_{\text {out }}=2 I_{1}^{\text {res }} \text { out }=I_{\text {in }} \frac{\delta_{c}{ }^{2}}{2\left(\delta_{c}+\delta_{o}\right)^{2}} .
$$

The second scheme therefore has two times the output intensity of the first with the same total resonator loss. The second scheme also reduces the detector coupling to the higher order modes of the resonator that have nodes in the mirror center. It can also be shown that if the output aperture is kept small relative to the mode size, which is always the case in our setup, Fabry-Perot mode structure is not effected and all the formalism derived for the mirrors without apertures can be used. ${ }^{56-58}$ Because of these reasons, the second scheme was the one we chose. 


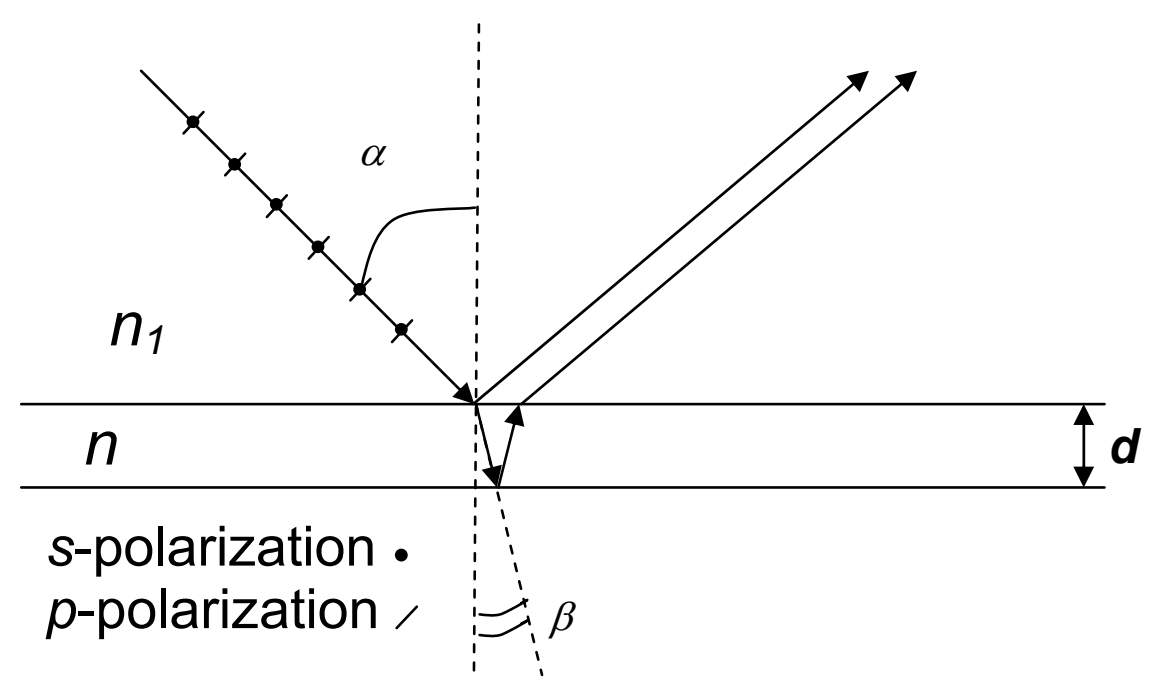

Figure 3.5: Dielectric coupling film.

For a dielectric film with the index of refraction $\mathrm{n}$ and the thickness $\mathrm{d}$, positioned at an angle $\alpha$ with respect to the radiation, propagating in the media with the index of refraction $n_{1}$ (Figure 3.5), the reflection coefficients for the $s$ and $p$ polarizations are given by

$$
\begin{gathered}
\delta_{c i}^{s, p}=\frac{4 r_{s, p} \operatorname{Sin}[x]}{\left(1-r_{s, p}\right)^{2}+4 r_{s, p} \operatorname{Sin}^{2}[x]} \\
r_{s}=\frac{\operatorname{Sin}^{2}[\alpha-\beta]}{\operatorname{Sin}^{2}[\alpha+\beta]}, \\
r_{p}=\frac{\operatorname{Tan}^{2}[\alpha-\beta]}{\operatorname{Tan}^{2}[\alpha+\beta]}, \\
x=2 \pi n v d \operatorname{Cos}[\beta],
\end{gathered}
$$

and

$$
\beta=\operatorname{ArcSin}\left[\frac{n_{1}}{n} \operatorname{Sin}[\alpha]\right]
$$


The notation $\delta_{c i}^{s, p}$ is used for the reflection coefficient because the beam splitter reflection coefficient acts as a coupling loss. With each pass the fraction $\delta_{c i}^{s, p}$ of the radiation gets reflected out of the resonator and lost.

Mylar film is a commonly used beam splitter material in the microwave because it is readily available, transparent in the optical region, and robust. It also contracts when heated above its production temperature to relieve the stress. This contraction is used to make high quality beam splitters by heating, with a heat gun, the Mylar film, fixed inside the beam splitter holder. The Mylar index of refraction in the microwave is $1.83 .^{59,60}$

As mentioned above, if the output coupling apertures are small, the Gaussian beam propagation theory results for the resonator without apertures can be used. ${ }^{56-58}$ For an aperture of radius $a$, the output coupling loss can be calculated from the equation (3.10) to be

$$
\delta_{c o}=1-\exp \left[-2\left(\frac{a}{\omega}\right)^{2}\right] \text {, }
$$

which for small $a$ becomes

$$
\delta_{c o} \approx 2\left(\frac{a}{\omega}\right)^{2} .
$$

Equation (3.1), written for the second coupling scheme, is now

$$
\delta_{t}=\delta_{r}+\delta_{d}+\delta_{c i}+\frac{1}{2} \delta_{c o}+\delta_{g}
$$

where $\delta_{r}, \delta_{d}, \delta_{c i}$ and $\delta_{c o}$ can all be estimated by using the appropriate equations presented above. 


\subsection{FREQUENCY VS. TIME DOMAIN MEASUREMENTS, MICROWAVE RINGDOWN}

\subsubsection{Fabry-Perot resonator frequency responce}

In the above discussion of the coupling schemes, equation (3.14) was presented for the resonance intensity. A derivation of the frequency response of the Fabry-Perot resonator, including the intensity at the resonance, is given below.

Consider the plane $A$ at the output coupling mirror, as shown in Figure 3.6. If $E_{\text {in }}$ is the radiation field intensity going into the cavity in the $\mathrm{TE}_{00}$ mode, then after the radiation reflects from the beam splitter and from the first mirror, the field at the plane $A$ just before the second mirror reflection is given by

$$
E_{A}^{(0)}=E_{i n} r_{b s} r_{m} t_{b s} \operatorname{Exp}\left(\varphi_{0}\right)
$$

where $r_{b s}=\sqrt{\delta_{c i}}$ is the reflection coefficient of the beam splitter, $r_{m}=\sqrt{1-\delta_{r}}$ is the mirror reflectivity coefficient, $t_{b s}=\sqrt{1-\delta_{c i}}$ is the transmission coefficient of the beam splitter, and $\varphi_{0}$ is the phase of the radiation field. After the first full pass, the radiation field at the plane $A$ is given by

$$
E_{A}{ }^{(1)}=E_{i n} r_{b s} r_{m} \exp \left(\varphi_{0}\right) t_{b s}{ }^{2} r_{m}{ }^{2} r_{c o} \exp (-\Delta \varphi)=E_{A}{ }^{(0)} t_{b s}{ }^{2} r_{m}{ }^{2} r_{c o} \exp (\Delta \varphi),
$$

where $\Delta \varphi=-\frac{4 \pi L}{\lambda}-$ the phase delay accumulated in one full pass of distance $2 L$ and $r_{c o}=\sqrt{1-\delta_{c o}}$ is the output coupling coefficient. 


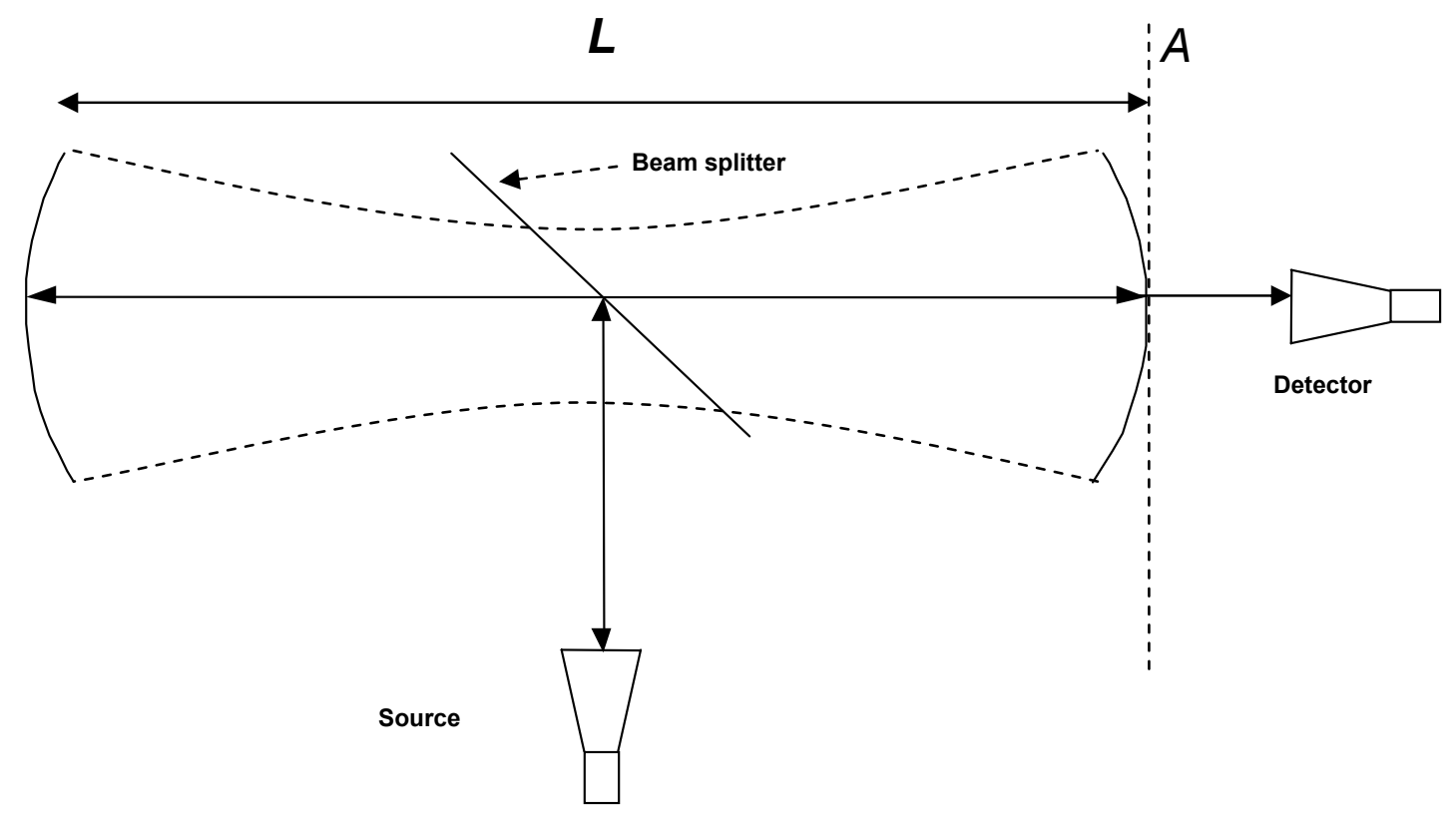

Figure 3.6: Fabry-Perot resonator block diagram.

After the $n$-th pass,

$$
E_{A}{ }^{(n)}=E_{A}{ }^{(1)}\left(t_{b s}{ }^{2} r_{m}{ }^{2} r_{c o} \exp (-\Delta \varphi)\right)^{n}=E_{A}{ }^{(1)} q^{n},
$$

where

$$
q=t_{b s}{ }^{2} r_{m}{ }^{2} r_{c o} \exp (\Delta \varphi)
$$

The total field intensity is the sum of all $E_{A}{ }^{(n)}$

$$
E_{A}{ }^{\text {total }}=\sum_{n=0}^{+\infty} E_{A}{ }^{(n)}=E_{A}{ }^{(1)} \sum_{n=0}^{+\infty} q^{n}=(|q|<1)=E_{A}{ }^{(1)} \frac{1}{1-q} .
$$


Taking into account the relations between the field intensity and the radiation intensity

$$
I_{A}=E_{A}^{\text {total }} E_{A}^{\text {total }}{ }^{*}
$$

and

$$
I_{\text {in }}=E_{\text {in }} E_{\text {in }}^{*},
$$

we can combine the equations (3.26)-(3.32) to derive the response of the resonator as a function of frequency:

$$
I_{\text {out }}=I_{A} \delta_{c o}=I_{\text {in }} \frac{\delta_{c i} \delta_{c o} T^{2}}{\left(1-2 T \operatorname{Cos}[\Delta \varphi]+T^{2}\right)},
$$

with

$$
T=\left(1-\delta_{c i}\right)\left(1-\delta_{r}\right) \sqrt{1-\delta_{c o}} \text {. }
$$

If $\delta_{c i}, \delta_{r}$, and $\delta_{c o}$ are all small $(<<1)$, equation (3.33) is simplified to

$$
I_{\text {out }}=I_{\text {in }} \frac{\delta_{c i} \delta_{c o}}{\left(1-2 T \operatorname{Cos}[\Delta \varphi]+T^{2}\right)} .
$$

Close to the resonance, $\operatorname{Cos}[\Delta \varphi] \approx 1-\frac{\Delta \varphi^{2}}{2}$ and equation (3.32) takes the form of the well known Lorentzian profile

$$
I_{\text {out }}=I_{\text {in }} \frac{a}{b+\Delta \varphi^{2}}
$$

$I_{\text {out }}$ is maximum when $\operatorname{Cos}[\Delta \varphi]=1$.

$$
I_{\text {out }}^{\max }=I_{\text {in }} \frac{\delta_{c i} \delta_{c o}}{\left(\delta_{r}+\delta_{c i}+\frac{1}{2} \delta_{c o}\right)^{2}}
$$


$I_{\text {out }}$ is minimum when $\operatorname{Cos}[\Delta \varphi]=-1$.

$$
I_{\text {out }}^{\min }=I_{\text {in }} \frac{\delta_{c i} \delta_{c o}}{4}
$$

with

$$
\frac{I_{\text {out }}^{\min }}{I_{\text {out }}^{\max }}=\frac{\left(\delta_{r}+\delta_{c i}+\frac{1}{2} \delta_{c o}\right)^{2}}{4} .
$$

Condition for the maximum output intensity is satisfied when $\Delta \varphi=2 \pi k$, where $k$ is an integer. The resonant frequencies are therefore

$$
v_{k}=k \frac{c}{2 L},
$$

with the frequency spacing between the two closest resonances

$$
\Delta v=\frac{c}{2 L}
$$

and the resonance full width half maximum $\delta v$ given by

$$
\frac{\delta v}{\Delta v}=\frac{\delta_{r}+\delta_{c i}+\frac{1}{2} \delta_{c o}}{\pi}=\frac{\delta_{t}}{\pi} .
$$

For $1 \%$ loss per pass $\left(\delta_{r}+\delta_{c i}+\frac{1}{2} \delta_{c o}=1 \%\right)$,

$$
\frac{I_{\text {out }}^{\min }}{I_{\text {out }}^{\max }} \sim 2.510^{-5}
$$

and

$$
\frac{\delta v}{\Delta v} \sim 3.210^{-3}
$$


so the response function consists of sharp Lorentzian-shaped resonances (fringes), separated by the Fabry-Perot free spectral range (FSR) $\Delta v$. The resonator loss can be calculated by using equation (3.42) if $\delta v$ and $\Delta v$ are measured from the resonator frequency response.

\subsubsection{Frequency domain measurements}

The derivation presented above explicitly required the radiation frequency to stay the same for all of the interacting wave fronts. In other words, equation (3.35) describes the response of the resonator, assuming the frequency of the electromagnetic radiation is swept infinitely slowly; so that at each frequency the system is given infinite time to reach the equilibrium. In real experiments, if the source frequency is scanned, at any given time a number of different frequencies are present inside the cavity, with amplitudes that depend on the scan speed. This limits the maximum possible scan speed if the resonance profile needs to be reconstructed without distortion. ${ }^{61}$

As an example, consider the time dependence of the output signal for the FabryPerot resonator with $\delta_{t}=1 \%$ and $L=10 \mathrm{~m}$. If the source of resonant frequency is turned on at the time $t=0$, it will take about $20 \mu S$ for the radiation to reach $90 \%$ of the final intensity. It is clear that if the source frequency is swept too fast, equation (3.35) is no longer valid and a different formalism has to be used.

Let us consider in detail what happens when the source angular frequency varies with time linearly as

$$
\omega(t)=\omega_{0}+d \omega \frac{t}{\tau}
$$

where $d \omega$ is defined by how much the frequency changes during the time 
$\tau=\frac{2 L}{c}$ it takes for the radiation to make a full pass. To simplify the computation, let us assume that at the time $t=0$ the source is resonant with the cavity, that is,

$$
\omega_{0} \tau=2 \pi
$$

For this case at any time $t$ the phase of the radiation is given by

$$
\varphi(t)=\varphi_{0}+\int_{0}^{t} \omega(t) d t=\varphi_{0}+\omega_{0} t+\frac{d \omega t^{2}}{2}
$$

Following the same steps as for the fixed frequency case with the only difference that the $\varphi_{n}(t)$ is now

$$
\varphi_{n}(t)=\varphi(t-n \tau)=\varphi_{0}+\omega_{0}(t-n \tau)+\frac{d \omega(t-n \tau)^{2}}{2}
$$

we can write the field amplitude at the detector mirror at the time $t$ as

$$
\begin{aligned}
& E_{A}(t)=E_{0} \sum_{n=0}^{+\infty} q \exp \left(i \varphi_{n}(t)\right)= \\
& =E_{0} \sum_{n=0}^{+\infty} q \exp \left(i\left(\varphi_{0}+\omega_{0} t-\omega_{0} n \tau+\frac{d \omega t^{2}}{2}-d \omega n \tau+\frac{d \omega(n \tau)^{2}}{2}\right)\right)
\end{aligned}
$$

Using equation (3.46), we can rewrite (3.49) as

$$
E_{A}(t)=E_{0} \exp \left(i\left(\varphi_{0}+\omega_{0} t\right)\right) \sum_{n=0}^{+\infty} q \exp \left(i\left(\frac{d \omega t^{2}}{2}-d \omega n \tau+\frac{d \omega(n \tau)^{2}}{2}\right)\right)
$$

Although this expression can not be reduced to a simple analytical form as it was done with equation (3.30), the sum can be truncated at high enough $\mathrm{n}$ and calculated numerically. The output intensity of the Fabry-Perot resonator at the time $t$ can now be calculated using (3.33) as

$$
I_{\text {out }}(t)=I_{A}(t) \delta_{c o}=E_{A}(t) E_{A}(t)^{*} \delta_{c o}
$$


Results of the numerical calculations are presented in Figure 3.7. To avoid the distortion of the resonator response discussed above, the frequency needs to be swept at a rate no greater than $\sim 0.1 \mathrm{GHz} / \mathrm{s}$, which corresponds to about 15 minutes scan of the full $90 \mathrm{GHz}$ range. For a comparison, the calculation was carried out and the results were compared with the experimental measurements for the scan rate of 30 $\mathrm{GHz} / \mathrm{s}$ (3 second full scan), routinely used in the FASSST system ${ }^{62}$.

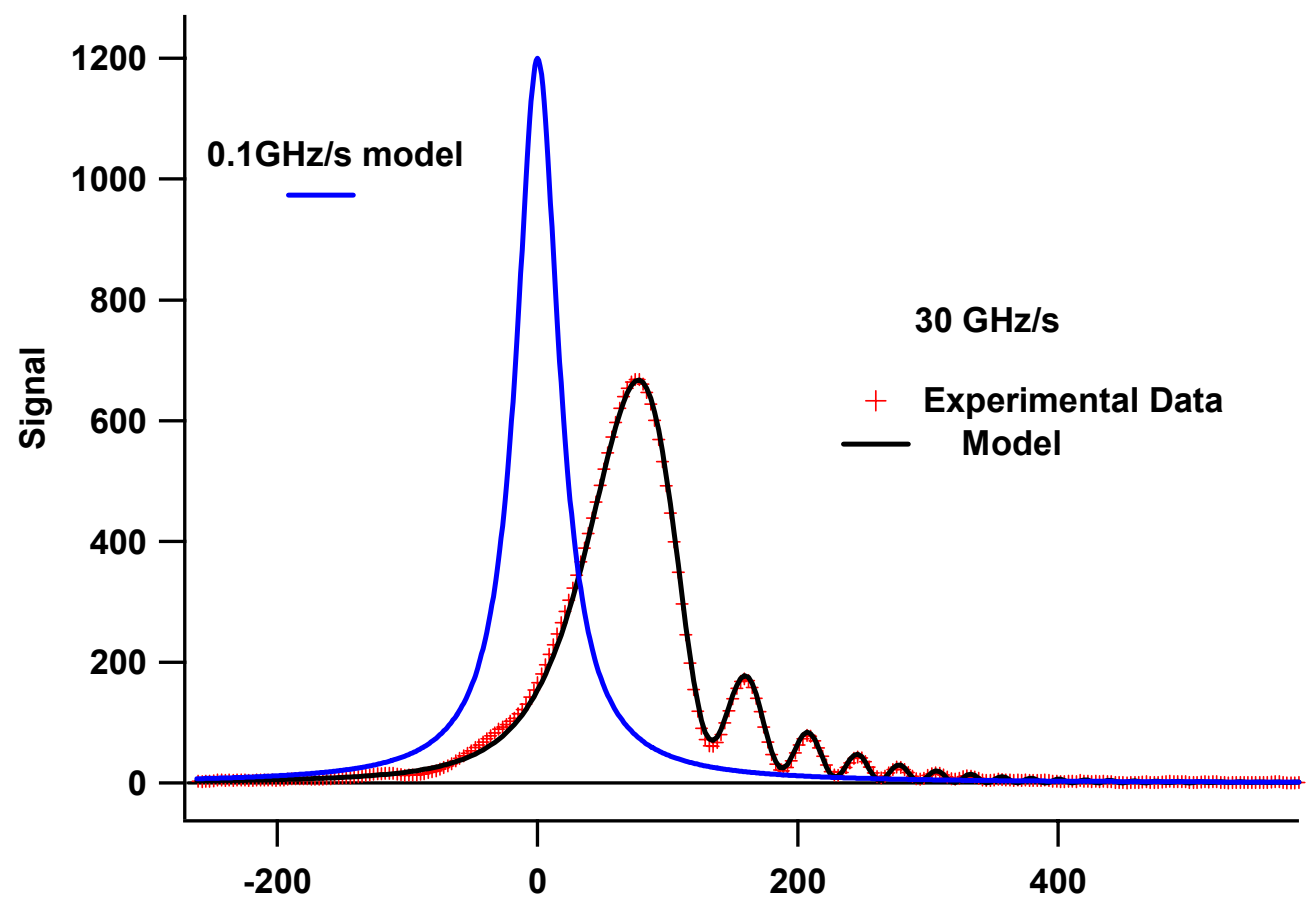

Figure 3.7: Resonator response as a function of frequency. Numerical computation results for $0.1 \mathrm{GHz} / \mathrm{s}$ and $30 \mathrm{GHz} / \mathrm{s}$. Experimental results for $30 \mathrm{GHz} / \mathrm{s}$, routinely used in the FASSST measurements.

Very good agreement of the experimentally measured resonator response with the model prediction led to the idea of using the details of the response to measure the resonator loss. Because of the small scale structure in the BWO frequency-voltage dependence and the time consuming numerical computations, this turned out to be 
impractical. The time was not spent in vain though, as while analyzing and discussing the roughly exponential periodic "ringing" of the resonator response, the idea of making the time domain measurements instead of frequency domain emerged.

\subsubsection{Time domain measurements, microwave ringdown}

The amount of the gas absorption is proportional to the mirror separation, making the long path advantageous. On the other hand, as shown above, as the mirror separation increases, the fundamental physics requires longer measurement times. This limits the maximum scan rate and restricts the number of possible absorption measurements in a given time period.

Another way of measuring the cavity Q is to measure the decay (ringdown) time of the photons inside the cavity. This ringdown time is directly related to the cavity loss via known system parameters. As for the derivation of the Fabry-Perot frequency response function, let us start with a single frequency. In this case if the input radiation is instantly turned off, the output radiation (and the detector signal) will not become zero at the same instant. The radiation that is already in the resonator will keep bouncing between the mirrors, decreasing in intensity with time because of the resonator losses. If $\delta_{t}=\frac{\Delta I}{I}$ is a fractional loss of the radiation intensity in a single pass ( $2 \delta_{t}$ - loss per round trip), then assuming $\delta_{t}<<1$, for a Fabry-Perot cavity of length $L$ :

$$
\frac{d I}{d t}=\frac{\Delta I}{L / c}=-I \delta_{t} \frac{c}{L},
$$


or

$$
I=I_{0} \exp \left(-\delta_{t} \frac{c}{L} t\right)=I_{0} \exp \left(-\frac{t}{\tau}\right)
$$

where

$$
\tau=\frac{L}{c \delta_{t}}
$$

By measuring the ringdown time $\tau$, one can readily obtain the resonator loss $\delta_{t}$ from (3.54). For a mirror separation $L$ of $10 \mathrm{~m}$ and a loss per pass $\delta_{t}$ equal to $1 \%$, the ringdown time $\tau$ is $\sim 3.3 \mu \mathrm{s}$. The above derivation is valid for any frequency, whether it is on or off the resonance. This makes it possible to measure resonator losses in time domain by measuring the ringdown decay times instead of retrieving the full frequency response function in frequency domain.

When frequency is scanned, at any time the radiation of varying frequencies is present inside the resonator. Because the $\delta_{t}$ is a slowly varying with frequency function, no matter what the radiation frequency is, the radiation intensity will decay according to the equation (3.53) if the input is turned off. This means that even for high speed scans where the standard frequency domain formalism is no longer valid, the decays stay exponential and the ringdown time does not depend on the speed of the scan or the place on the resonance curve. Making time domain ringdown measurements therefore presents a way to avoid the limitations on the scan speeds. 
Numerically the radiation intensity time dependence can be calculated by modifying equation (3.50). If the input signal is turned off at a time $t$, the summation needs to start from the $n=\frac{t}{\tau}$ instead of $n=0$ to give

$$
E(t)=E_{0} \exp \left(i\left(\varphi_{0}+\omega_{0} t\right)\right) \sum_{n=\frac{t}{\tau}}^{+\infty} q \exp \left(i\left(\frac{d \omega t^{2}}{2}-d \omega n \tau+\frac{d \omega(n \tau)^{2}}{2}\right)\right)
$$

If the signal is not turned off but instead the frequency is instantaneously changed in a way that the new frequency is now far from the resonance, the result for low loss resonator will be virtually the same. Indeed, because the intensity away from the resonance is negligible compared with the intensity at the resonance (equation (3.43)), jumping in frequency space away from the resonance will lead to the exponential decay to the non resonant intensity, which is close to zero.

Assuming for simplicity that the new frequency is exactly in the middle between the two consecutive resonances, the resonator response can be modeled with the following equation

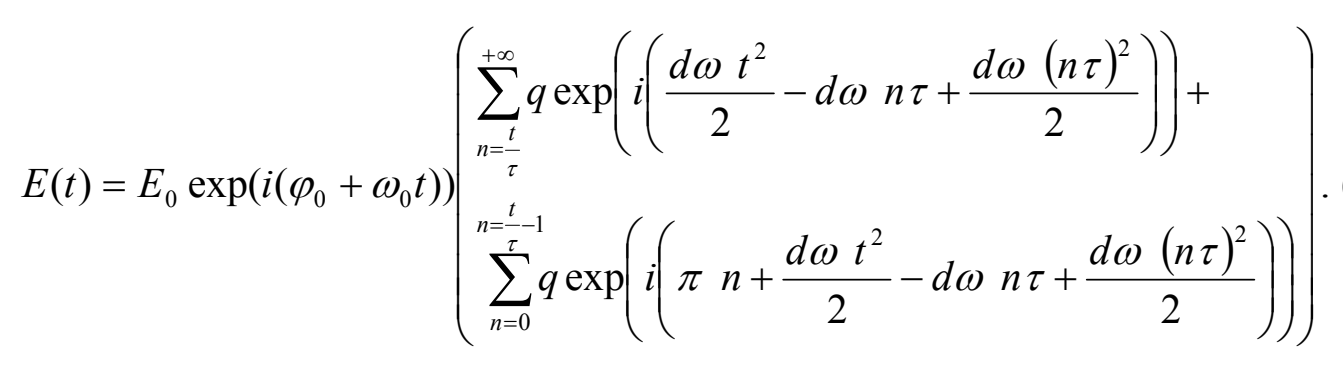

Numerical simulations show that the ringdown time to high order does not depend on the exact place that the ringdown starts on the resonance curve, or the scan speed. This was later checked and confirmed experimentally. Details of the experimental fulfillment of the time domain ringdown measurement idea are described in the following chapter. 


\subsection{SYSTEM OVERVIEW}

\subsubsection{The basic system}

The block diagram of the system is shown in Figure 3.8. The system includes a microwave radiation source, 10-meter Fabry-Perot resonator cavity, liquid helium cooled InSb bolometer and the necessary electronics. The microwave radiation source is an ISTOK OB-66 (former OB-24) backward wave oscillator (BWO) tube, covering the $170-260 \mathrm{GHz}$ frequency range. Similar tubes are available from ISTOK that cover the $\sim 100-1000 \mathrm{GHz}$ range. An electromagnet is used to provide the uniform magnetic field required for the tube operation. Two wire grid polarizers rotated at $\pm 45^{\circ}$ relative to each other and a Faraday rotator provide a quasi-optical isolator that minimizes the radiation coupling back to the source. The Fabry-Perot resonator is coupled with the radiation source via a 0.5 mil Mylar beam splitter and with the InSb detector via a $1 / 8^{\prime \prime}$ diameter iris in one of the mirrors. The detector signal is amplified with a QMC ULN95 low noise wideband preamplifier and then is used as an input to the SRS DG535 pulse generator and a National Instruments data acquisition board with a $5 \mathrm{MHz}$ sampling rate, which is used to transfer data to a computer for subsequent processing. 


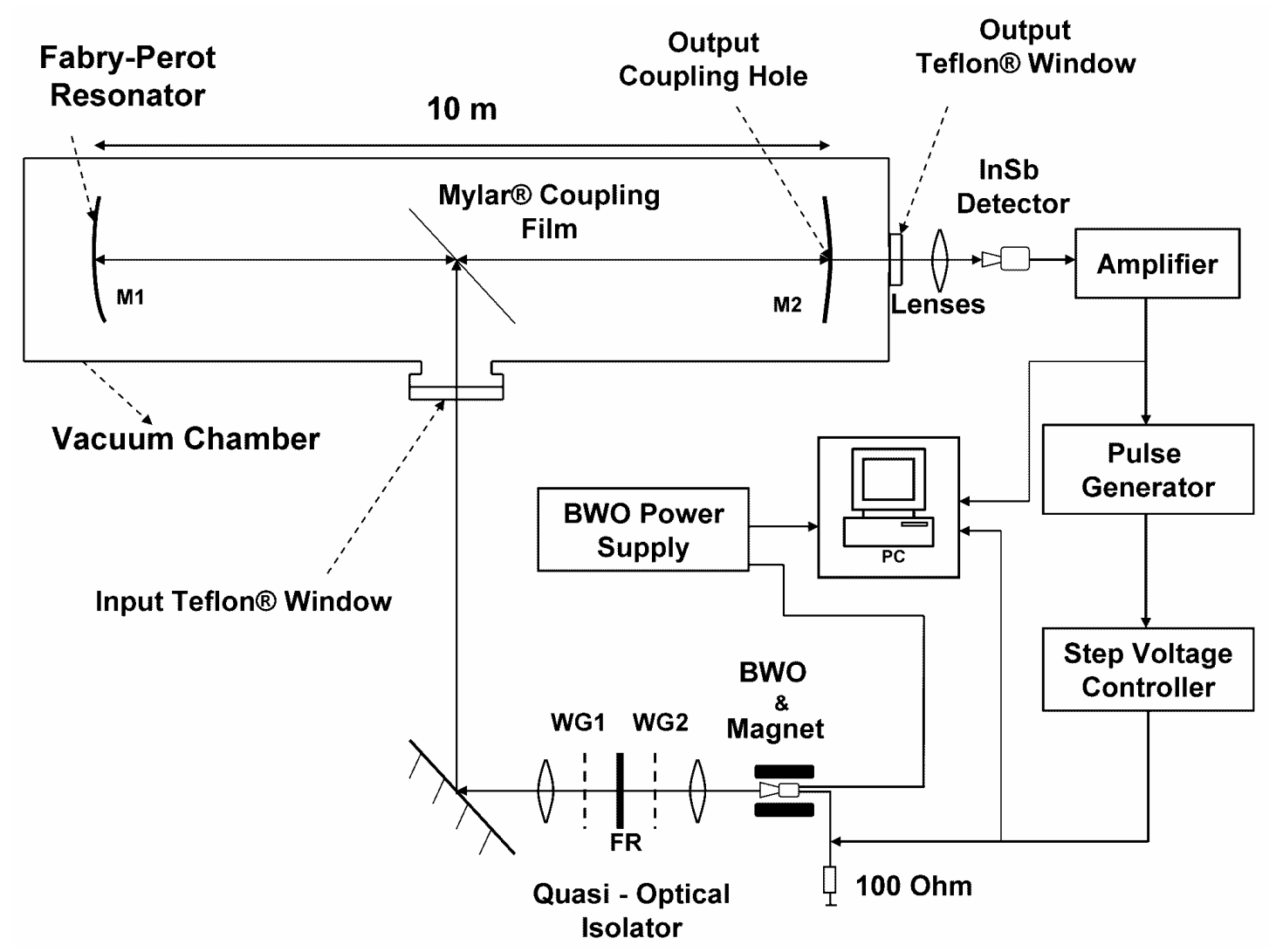

Figure 3.8: Block diagram of the experimental setup.

\subsubsection{Fabry-Perot resonator}

The Fabry-Perot resonator mirrors are constructed from oxygen-free high conductivity (OFHC) copper, have 12" $(30.5 \mathrm{~cm})$ diameters, $25^{\prime}(762 \mathrm{~cm})$ radii of curvature, and are separated by $\sim 10$ meters. The free spectral range (FSR) of this resonator is $15 \mathrm{MHz}$, which leads to about 6000 resonances in the $170-260 \mathrm{GHz}$ frequency range. This $15 \mathrm{MHz}$ spacing between available measurements is adequate for all the relevant atmospheric pressures since typical pressure broadening parameters are of the order of a few MHz/Torr. 
As mentioned above, the total fractional radiation intensity loss per pass $\delta_{t}$ can be written as a sum of five terms

$$
\delta_{t}=\delta_{r}+\delta_{d}+\delta_{c i}+\frac{1}{2} \delta_{c o}+\delta_{g}
$$

where $\delta_{r}$ is the reflection loss, $\delta_{d}$ is the diffraction loss, $\delta_{c i}$ is the Mylar splitter coupling loss, $\delta_{c o}$ is the output hole coupling loss, and $\delta_{g}$ is the gas absorption loss. Gas absorption can therefore be measured by subtracting the losses with and without the gas in the cavity, provided all other losses stay the same. However, these losses do change with the gas fill. The beam splitter coupling coefficient, the resonator mode size and therefore output coupling, and the diffraction loss all change as the gas index of refraction changes. These changes, although small, are an example of the systematic effects that can hinder the study of the underlying physics.

The total loss of the empty resonator is shown in Figure 3.9. It is below $1 \%$ per pass $\left(1 \times 10^{-5} \mathrm{~cm}^{-1}\right)$ for the whole frequency range, translating to a resonator quality factor of 5 to 7 million. Diffraction losses can be seen at the low frequency end of the scan. The periodic structure is the result of interfering stray reflections, including detector reflections and possibly low-incidence chamber wall reflections, which modulate the coupling loss coefficients of the cavity. Because we make measurements at many frequencies, this systematic effect is revealed and can be considered in the gas absorption analysis. 


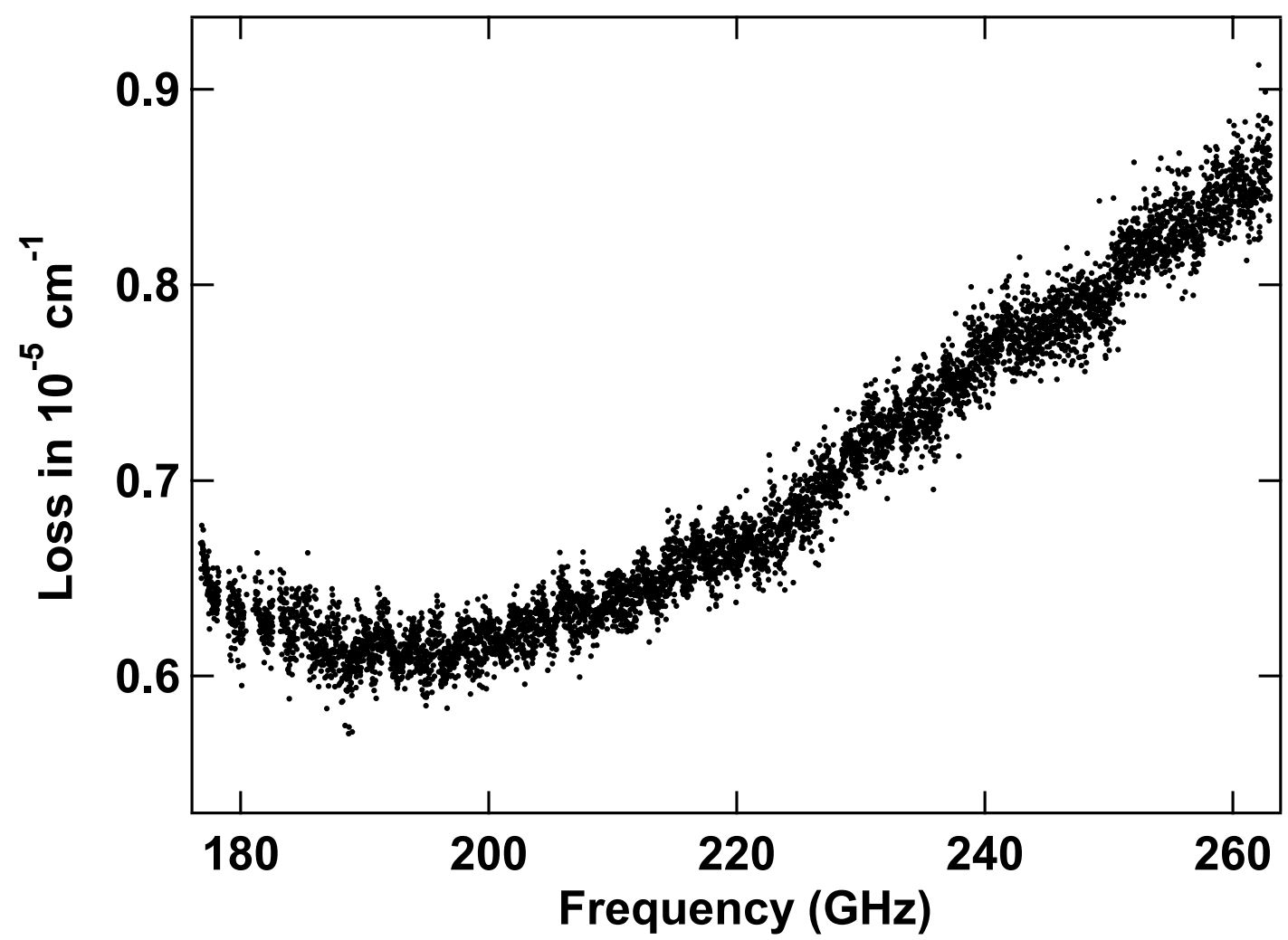

Figure 3.9: Empty resonator loss per pass as a function of frequency. Single scan, taking about 3 seconds and containing $\sim 6000$ ringdowns is shown. For the $10 \mathrm{~m}$ mirror separation, absorption of $10^{-5} \mathrm{~cm}^{-1}$ is equivalent to $1 \%$ loss per pass.

Figure 3.10 shows the measured resonator loss per pass along with the contributions from the Mylar beam splitter, output coupling aperture, mirror reflection and the diffraction loss, calculated using the appropriate equations presented in the section 3.2 and the system parameters given above. Calculated total loss is almost two times smaller than the measures loss. Measured diffraction loss at the low frequency end of the scan is also greater than predicted. We expected the main reason for the greater than predicted measured loss to be the greater mirror reflection loss than given by equation (3.2). It has in fact been suggested ${ }^{63}$ that the predicted by equation (3.2) reflection loss should be roughly doubled to give a 
better agreement with the experimental results for a smooth mirror surface. Surface roughness only further reduces the reflectance. ${ }^{64}$ To check if it was indeed the main reason for the above disagreement, we have performed a number of tests to get a better understanding of other resonator losses.

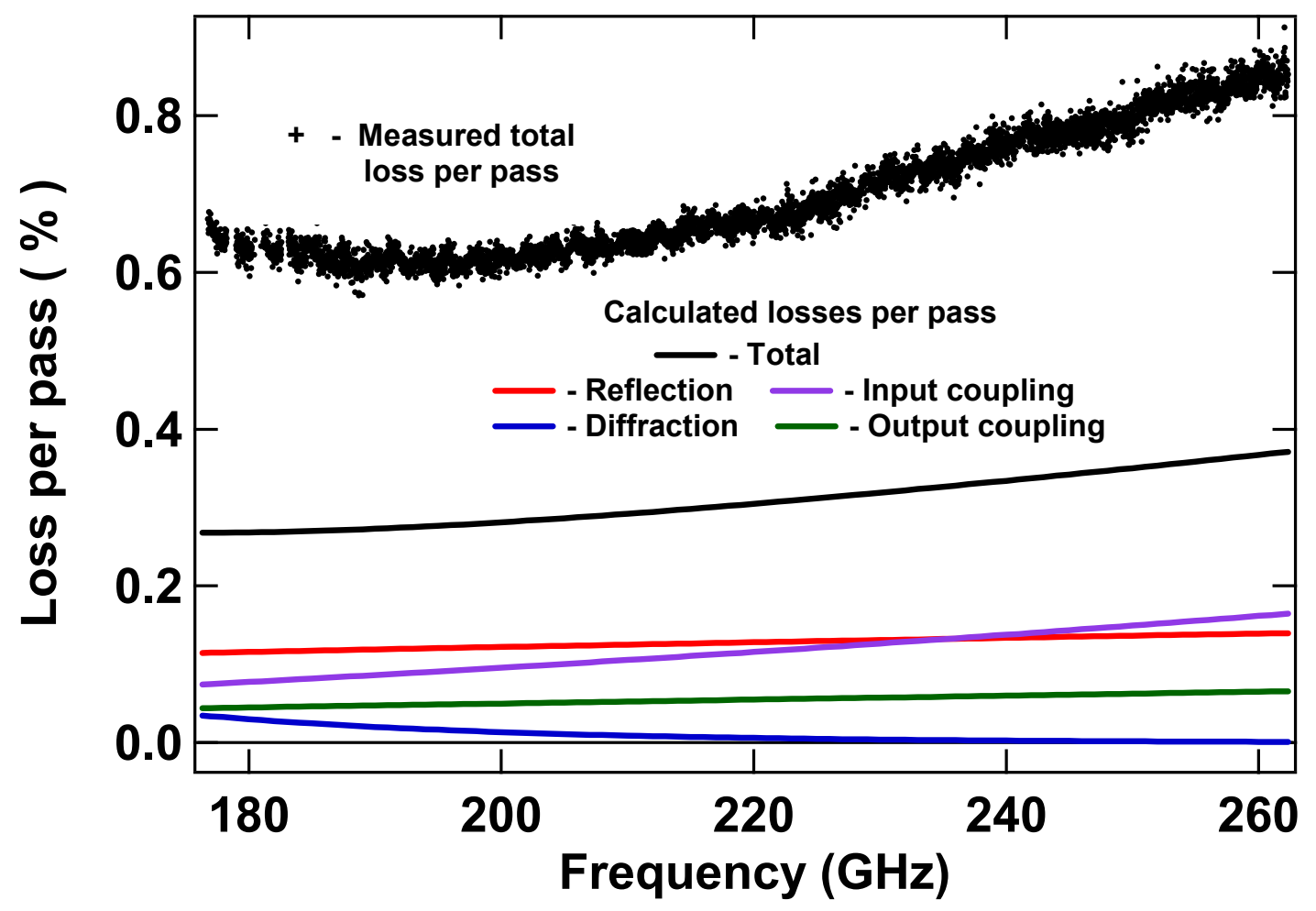

Figure 3.10: Measured resonator loss per pass comparison with the calculated losses. Calculated reflection, diffraction, input and output coupling losses are shown along with the total calculated loss and the total measured loss.

By using the EchoSorb ${ }^{\circledR}$ masks, effectively reducing the mirror diameters, and measuring the diffraction losses as a function of the open mirror area, we have concluded that the mode sizes are not the same on the two cavity mirrors. The best agreement between the measured results and theoretical calculations is achieved if mirror radii are taken to be $\sim 22^{\prime}$ and $\sim 24^{\prime}$ for the detector mirror and the mirror 
without the aperture, respectively. The mirrors were made in the Ohio State University machine chop from the rolled stock of 1 " thick copper. Considering the large diameters of the mirrors and the long curvature radii, it is not surprising that the actual mirrors curvatures can vary. Because we use mirror curvatures quite smaller than $33^{\prime}$ that would make mirror configuration confocal, mode sizes do not heavily depend on the mirror curvature, and curvature variations mentioned above are not critical. For the the $\sim 22^{\prime}$ and $\sim 24^{\prime}$ curvatures, the output coupling loss will also increase by about $10 \%$ for the same aperture.

The beam splitter coupling coefficient was estimated by inserting an extra beam splitter into the cavity and measuring the increase in the cavity loss. Results were about $50 \%$ higher than what is predicted by equation (3.18). Possible reason for this disparity is the difference between the actual Mylar thickness or the index of refraction, and the numbers used in the calculations.

We can now estimate the mirror reflectivity losses by subtracting the Mylar beam splitter coupling loss, diffraction loss and the output coupling loss from the total measured loss of the resonator. The result is shown in Figure 3.11 along with the fit of the $200-270 \mathrm{GHz}$ region to the square root frequency behavior. Deviation from the theoretical square root frequency behavior is probably an indication of the accuracy of other system loss prediction. Possible reason for the disagreement at the lower frequency end is that the diffraction loss frequency behavior is not well described by equation (3.11) due to deviations of the mirror surfaces from an ideal spherical form. As expected, the measured reflectivity loss is greater than what is predicted by the Hagen-Rubens relation. 


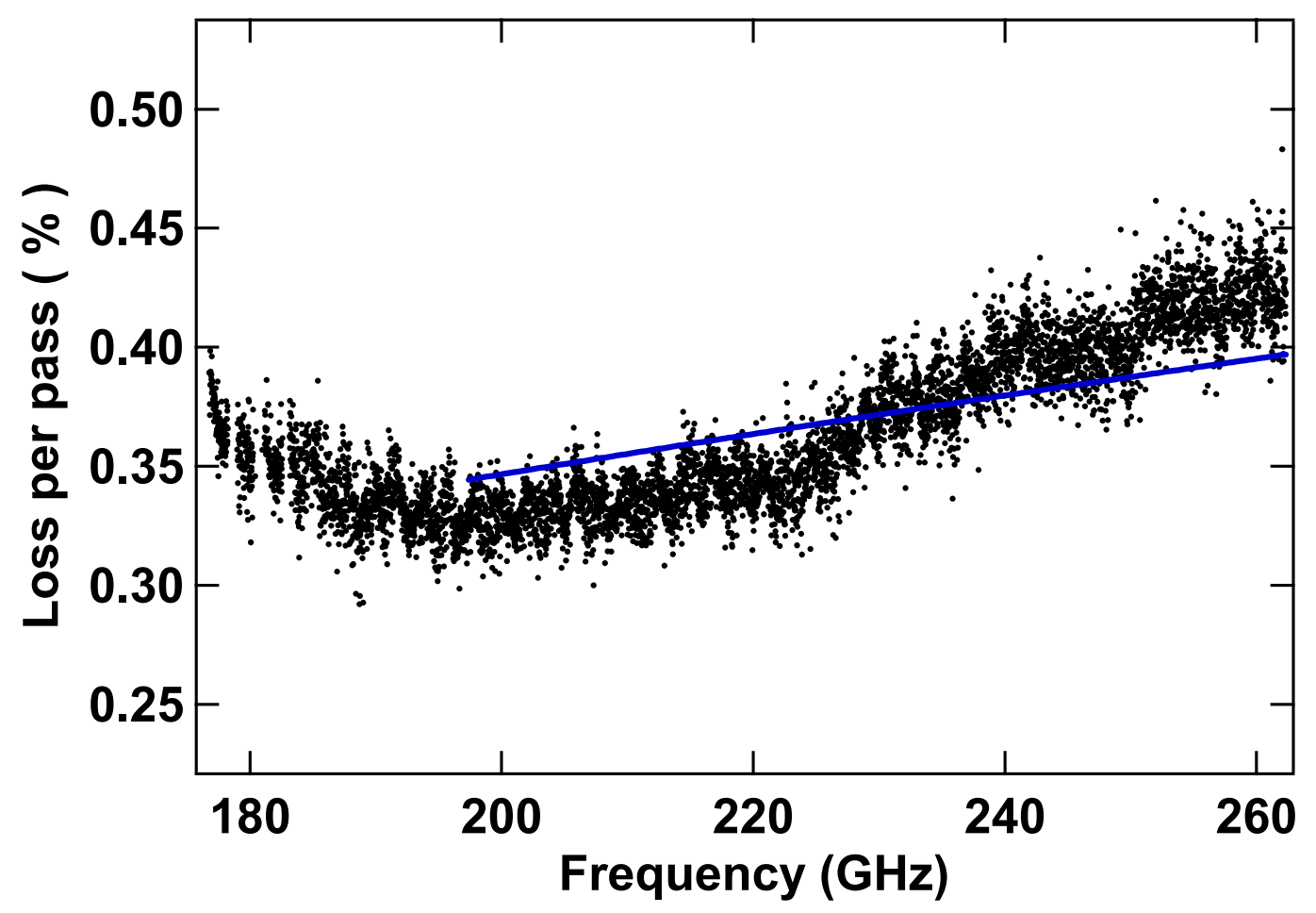

Figure 3.11: Mirror reflectivity loss, calculated by subtracting the Mylar coupling in, the aperture coupling out and the diffraction losses from the measured total resonator loss per pass. Solid line is a fit of the $200-270 \mathrm{GHz}$ range to a square root frequency behavior.

\subsubsection{Pressure and temperature effects}

The resonator is enclosed in a Grade 304 stainless steel vacuum chamber that can be pumped down to below $10^{-5}$ Torr with a diffusion pump and is certified for pressures up to $10 \mathrm{~atm}$. Teflon windows are used for the microwave radiation. The vacuum chamber is inside a thermally insulated enclosure made of 1" HT-300 polyisocyanurate foam insulation by HiTHERM ${ }^{\circledR}$ with operating temperature range of -183 to $+149{ }^{\circ} \mathrm{C}$. The temperature inside the enclosure can be controlled between $-80{ }^{\circ} \mathrm{C}$ and $+100{ }^{\circ} \mathrm{C}$ with temperature variations along the chamber of less than \pm 1 ${ }^{\circ} \mathrm{C}$ and with the average temperature varying by less than $\pm 0.5{ }^{\circ} \mathrm{C}$ during the experiment. This temperature range covers all the relevant atmospheric temperatures. 
Because the vacuum chamber is heated and cooled by heating or cooling the air inside the insulating enclosure, four fans are located inside the enclosure to insure the quick air turnover for the necessary convection. The air is heated with three sets of heating tapes at the bottom of the insulating enclosure. To cool the chamber, liquid nitrogen is flown through the cryogenic pipes, finned to improve the heat exchange. To get an idea of the liquid nitrogen consumption requirements, let us note that evaporating and heating up to the room temperature about 2.2 liters of liquid nitrogen will cool the chamber down by 1 degree assuming no heat losses. The lowest temperature we were able to reach with this cooling setup is about $-80{ }^{\circ} \mathrm{C}$. Figure 3.12 shows the chamber temperature as a function of time for this $-80{ }^{\circ} \mathrm{C}$ cool down run. The time constant of the insulating enclosure is $\sim 7$ hours.

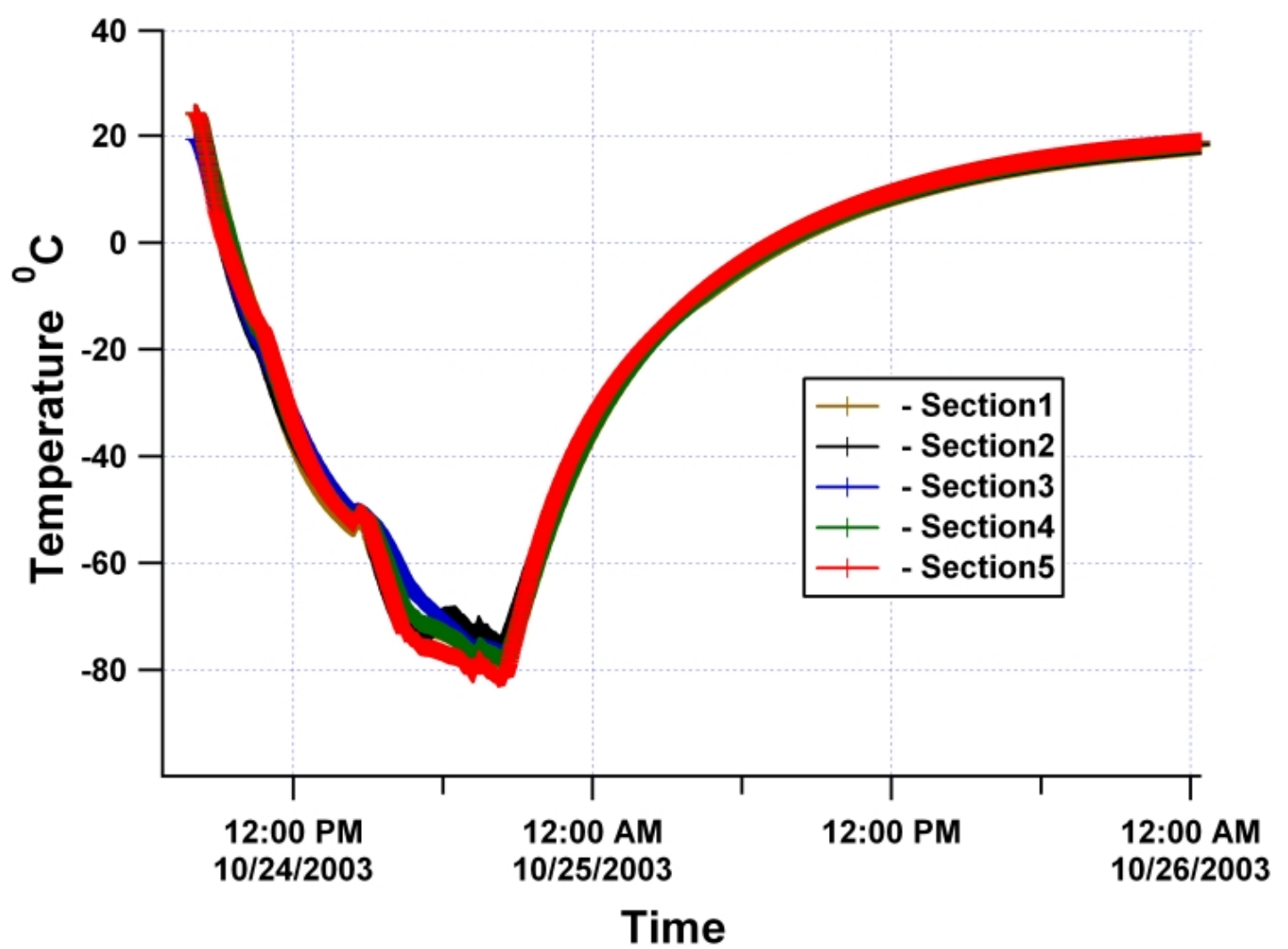

Figure 3.12: Temperatures of the five chamber sections during the cool down to about $-80{ }^{0} \mathrm{C}$ and the chamber return to the room temperature after the nitrogen cooling was turned off. 
Uncertainty in the mirror separation measurement will directly effect the resonator loss measurements as can be seen from equation (3.54). For each temperature, the mirror separation of the empty resonator was calculated based on the measured resonator free spectral range (FSR), using the equation (3.41). The FSR was determined from the frequency calibration based on the five water isotopes line frequencies, as discussed in the next section. Figure 3.13 shows $L$ as a function of temperature. $L$ increases linearly with temperature as

$$
L(m)=10.33337(16)+0.000161(5) t(C)
$$

From this, the linear coefficient of thermal expansion (CTE) of our chamber can be calculated to be15.6(5) $\mu \mathrm{m} / \mathrm{m}^{\circ} \mathrm{C}$. This result is in agreement with the literature temperature expansion coefficient for the 300 series SS, which is $14-19 \mu \mathrm{m} / \mathrm{m}^{\circ} \mathrm{C}$.

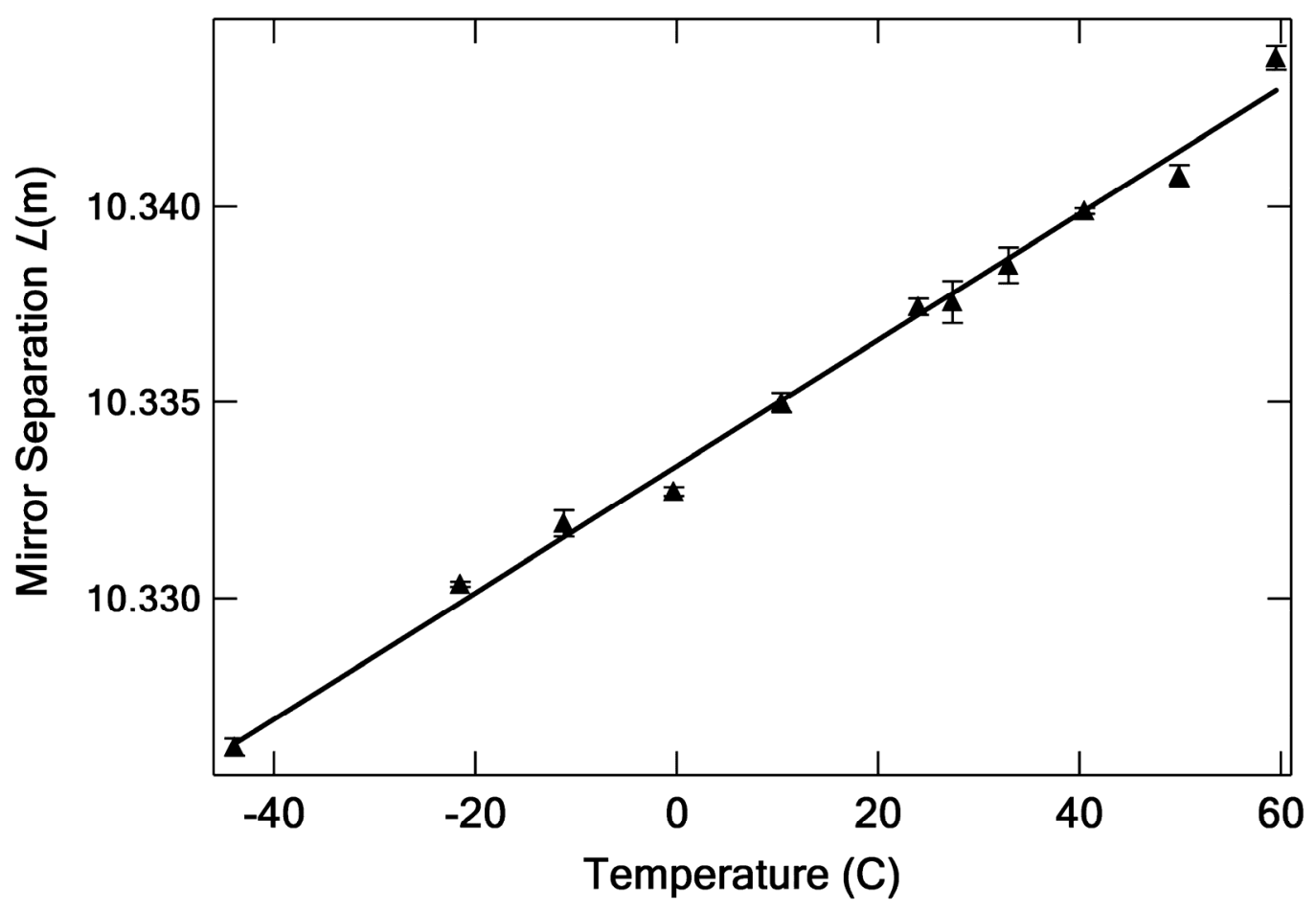

Figure 3.13: The empty resonator mirrors separation as a function of temperature, calculated from the measured resonator free spectral range. Solid line is a linear fit of the data. 
The empty resonator frequency is always calibrated at low (1-2Torr) water pressures. Mirror separation can be easily estimated for the cavity filled with the gas at atmospheric pressures. Indeed, using the stainless steel modulus of elasticity $E=195 \mathrm{GPa}$, the chamber wall thickness $r=1 / 4$ " and the chamber diameter $d=16$ ", we can calculate that for the gas pressure $P$ equal to $3 \mathrm{~atm}$,

$$
\Delta L=L P \frac{A_{\text {end }}}{A_{\text {wall }}} \quad E \approx 0.2410^{-3} \mathrm{~m}=0.24 \mathrm{~mm}
$$

where $A_{\text {end }}=\frac{\pi d^{2}}{4}$ is the end plate area, $A_{\text {wall }}=\pi d r$ is the area of the chamber wall cross section. This has negligible effect on the absorption measurement $\left(\frac{\Delta \alpha}{\alpha} \approx \frac{\Delta L}{L} \approx 2.310^{-5}\right)$ and only needs to be taken into account if the accurate frequency calibration is required for higher pressures. 


\subsection{EXPERIMENTAL PROCEDURES}

The frequency scan strategy is similar to the one used in the FAst Scan Submillimeter Spectroscopy Technique (FASSST) system. ${ }^{62,} 65$ Unlike optical ringdown systems which inject an amplitude modulated pulse into a cavity resonant in frequency with several optical modes, we inject a pulse of microwave photons into each cavity mode by rapidly sweeping a CW source across the frequency response of the cavity. To accomplish this, we use a free running BWO tube and scan the frequency by changing the voltage applied to the tube. The response as a function of frequency for the Fabry-Perot resonator is a well-known function (3.35), with resonances separated by the free spectral range of the resonator $(15 \mathrm{MHz}$ in our experiment). In order to provide a well defined start to the ring down process, a step function in voltage is applied to the BWO while its frequency is within each resonance. This changes the BWO frequency to a frequency which is not supported by a Fabry-Perot mode and the ring down process commences. To do this, we first use the detector signal to trigger a pulse generator. The signal from the pulse generator, modified by the step voltage controller (SVC) is then applied across a 100 ohm resistor to the BWO ground. As the detector signal approaches the set trigger level, the SVC generates a voltage pulse that is added to the high voltage ramp to jump, in frequency space, away from the resonance frequency, as shown in Figure 3.14. Because of the low loss of the resonator, this is essentially equivalent to switching the source off instantaneously (much faster than the cavity ringdown time), as described in section 3.3. The same step is repeated for all of the resonances. Because the ringdown time to high order does not depend on the exact place of the trigger on the resonance curve, scan speed or trigger level; the resulting system is 
very robust and not sensitive to changes in the above parameters. This makes it possible not to phase lock the source and to use the free running broadband BWO tube at high sweep rates.

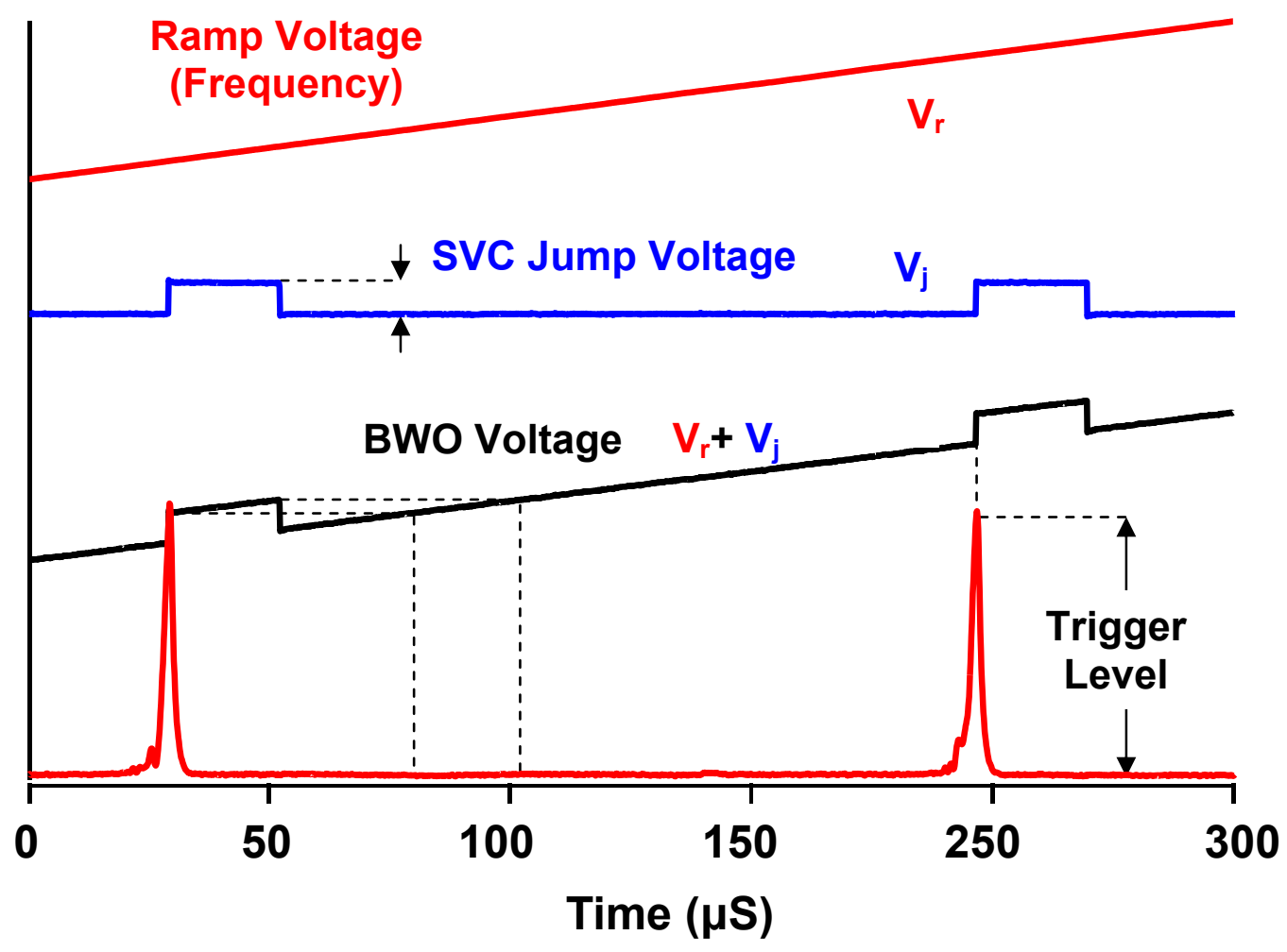

Figure 3.14: Triggering scheme. The sum $\mathrm{V}_{\mathrm{r}}+\mathrm{V}_{\mathrm{j}}$ of the ramp voltage and the SVC jump voltage is applied to the BWO, resulting in a rapid frequency jump of the source frequency.

Because the small scale voltage-frequency characteristics of BWO tubes are highly nonlinear, ${ }^{62}$ the SVC is required to choose the size of the voltage step for each resonance. This is especially important because of the higher order modes of the Fabry-Perot cavity, located in between the $\mathrm{TEM}_{00}$ mode resonances. The detector signal for a small part of the scan, demonstrating the variation in the mode amplitude and voltage spacing, as well as the higher order modes of the resonator cavity, is shown in Figure 3.15. A constant step voltage would cause, for some resonances, 
the source frequency to jump to the region of a higher order mode, interfering with the ringdown time measurement. The voltage step, generated by the SVC, is based on the time separation between the preceding FP modes and is tuned to make the jumps constant in frequency, not in voltage, allowing the source frequency to always jump to the point where the FP signal is virtually zero.

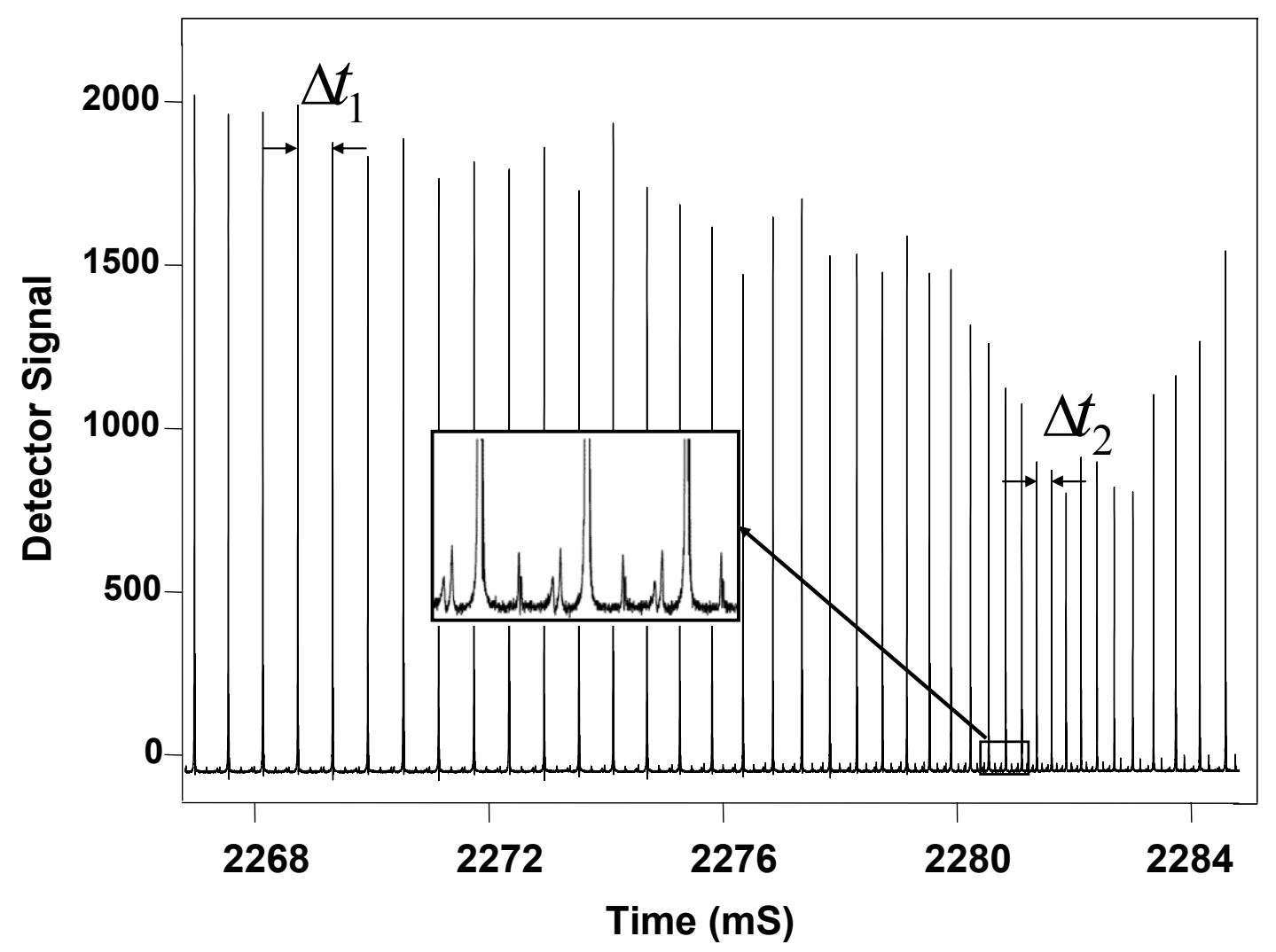

Figure 3.15: Typical amplitude and mode voltage separation variations of the resonator modes. Higher order FP modes are clearly visible, especially at the high frequency end of the scan.

It takes about 3 seconds in a typical scan to sweep the full tube frequency range of $\sim 90 \mathrm{GHz}$. Three channels are recorded with a $5 \mathrm{MHz}$ DAQ board during a scan: the detector signal, the trigger signal, and the sweep voltage. Because of the intrinsic BWO output radiation changes, the multi-spatial mode output and the beam steering, 
the detector signal can vary by more than a factor of 10 for different resonance frequencies. This dictates the need to adjust the trigger level to compromise between the number of resonances without a trigger and the signal strength of the triggered ones. The trigger level also has to be greater than the largest signal of the higher order resonator modes. Otherwise these modes will interfere with the SVC generation. In a typical scan we miss about $2 \%$ of the points, mostly at low frequency, where the BWO signal is weaker. If needed, all points can be recovered by lowering the trigger level. In practice though, the phenomena we study change slowly with frequency, and in most cases we significantly oversample the frequency space.

In the next step, each ringdown is fit to an exponent to measure the ringdown time. These ringdown times are used to calculate the system loss by using the equation (3.54) and the final result is a table of the resonator loss vs. the resonance (fringe) number. By adding a few torr of a gas with known transition frequencies to the empty cavity, we can calibrate our frequency. Subtraction of the empty resonator loss from the loss of the resonator filled with 1 Torr of water vapor is shown in Figure 3.16. In principle, two lines are enough to find both FSR and an absolute frequency value of the first resonance, thus defining all of the resonance frequencies. Typically five lines of water and water isotopes are used to calibrate the empty cavity frequency to below $\mathrm{MHz}$ accuracy. As will be discussed later, filled cavity frequency is calibrated by comparing its BWO small-scale structure with that of an empty cavity. 


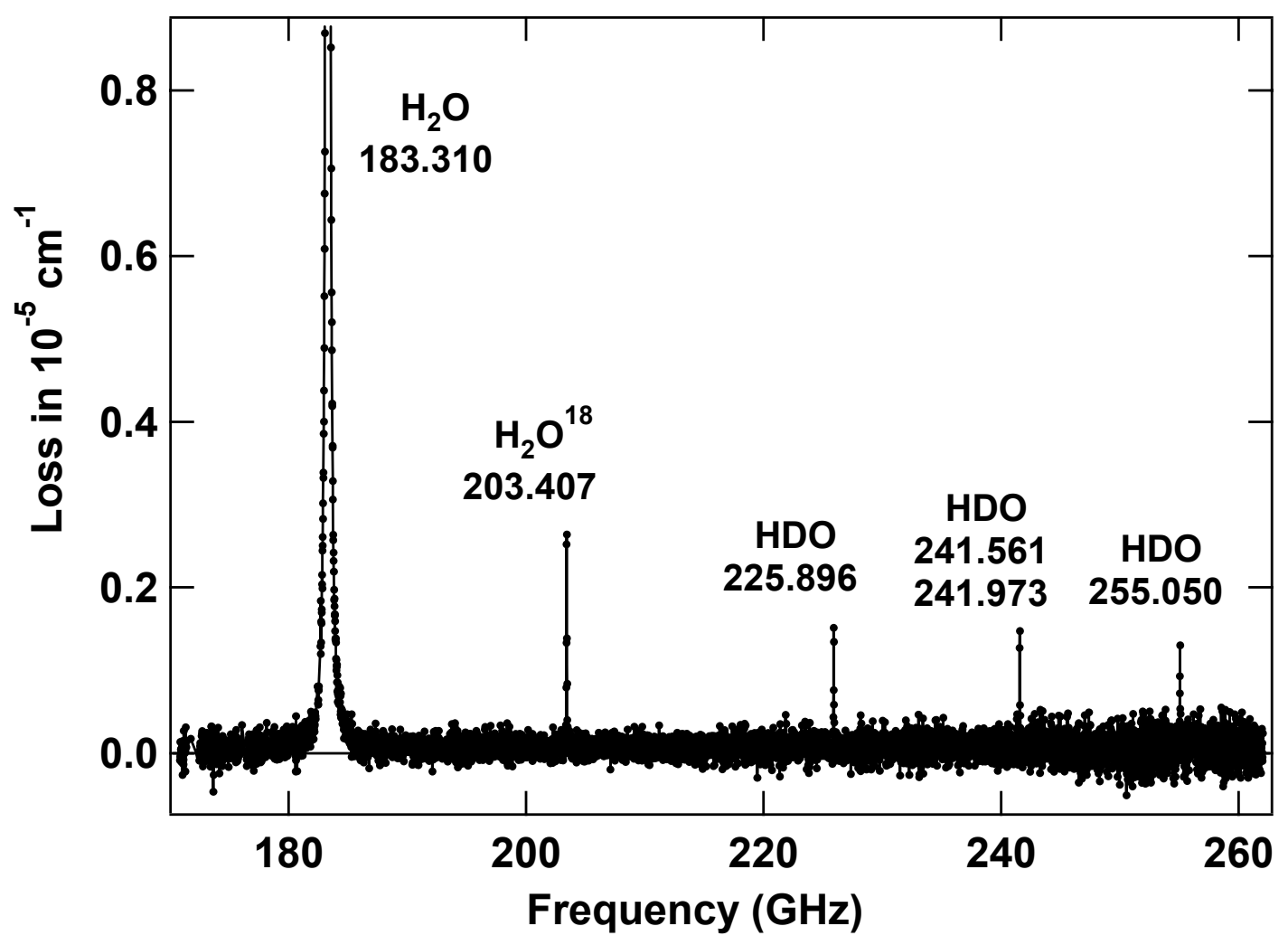

Figure 3.16: Absorption of 1 Torr of water vapor. The five lines used for the frequency calibration are marked.

Because a single scan only takes a few seconds, many scans are taken during a typical day. We normally start by measuring the loss of the empty resonator, then take data as we add gases to the chamber. After reaching the desired final pressure, the system is monitored over a period of time to make sure that all the non-equilibrium processes related to the gas fill stop. Data is taken at this final pressure and at multiple lower pressures as the gas is pumped out. Finally, many scans of an empty cavity are taken. 


\section{CHAPTER 4}

\section{RESULTS}

\subsection{EMPTY RESONATOR LOSS}

Empty resonator loss as a function of frequency was shown above in Figure 3.9. Figure 4.1 shows the subtraction of two consecutive scans and two groups of 50 scans, averaged. Although the statistical uncertainty of the fits to the individual exponential decays is below $4 \times 10^{-8} \mathrm{~cm}^{-1}$, the full scan absorption scatter shown in Figure 4.1 is about five times greater. If the trigger level is kept constant, both individual uncertainty and scan-to-scan scatter increase with frequency, mostly due to the increase of the radiation intensity and the resultant triggering further away from the resonance center frequency. This scatter can be reduced dramatically by increasing the effective integration time from $\sim 10^{-6}$ seconds by simple averaging of consecutive scans, as can be seen from the Figure 4.1. However, it was expected and quickly confirmed by the experimental data, that the limiting factor for the accurate measurement of the absorption is not the precision of the individual measurements but rather the error introduced by the systematic effects. Because of this, the system was intentionally designed to measure many modes with the short integration time, allowing for quick and effective means to discover and account for the systematic effects. 


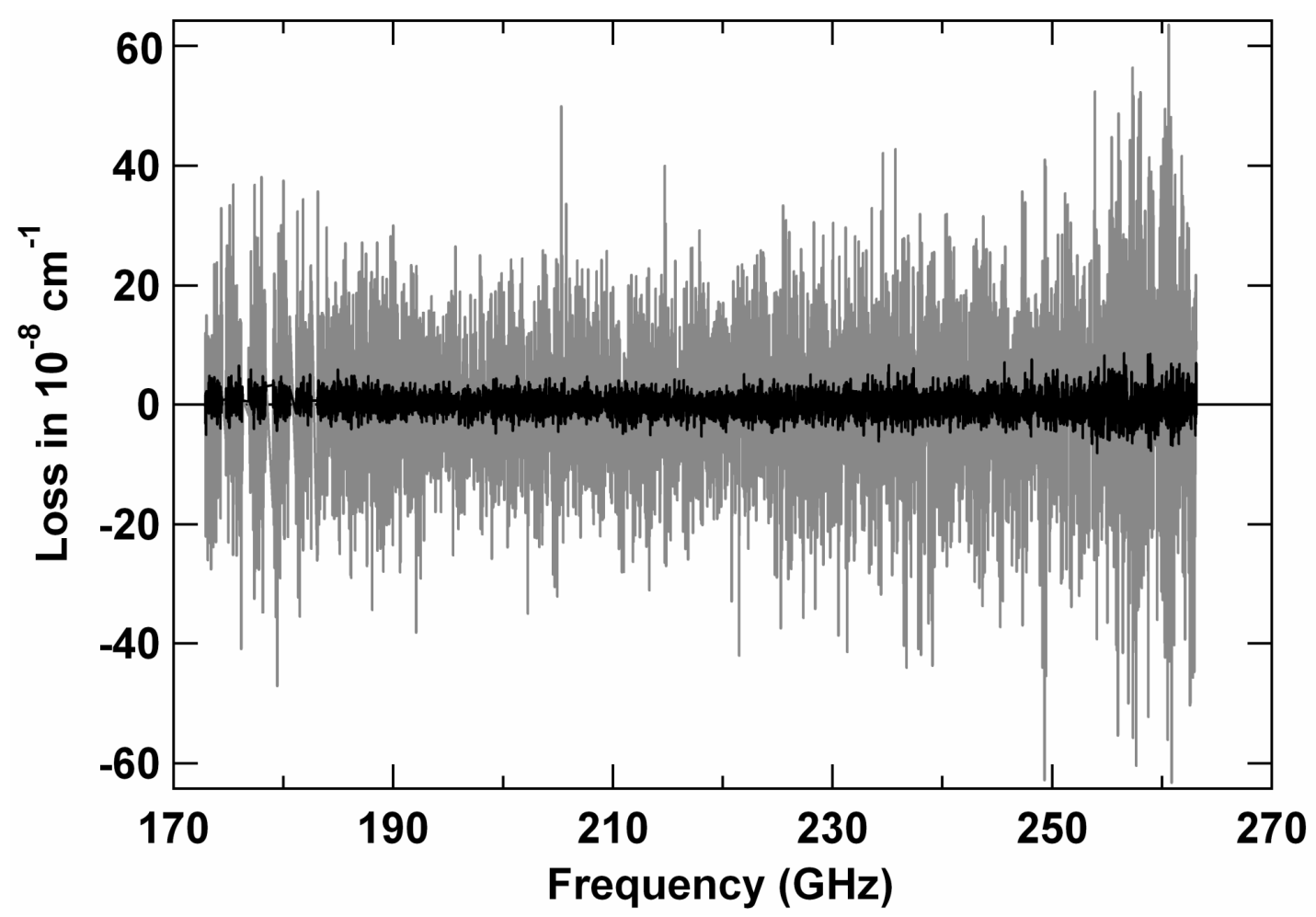

Figure 4.1: Subtraction of two consecutive scans (grey) and two groups of 50 scans, averaged (black), of the empty resonator.

Figure 4.2 shows 50 scans, averaged and processed using Fourier Transform (FT) techniques to eliminate the sharp (every other Fabry Perot fringe) baseline variations introduced by reflections from the input Teflon window. The lower frequency region with missing points (up to $\sim 182 \mathrm{GHz}$ ) was excluded to carry out the Fourier Transformation. Residual periodic structure in the measured loss can still be clearly seen. This structure, as mentioned above, is a result of stray reflections from the surfaces other than the cavity mirrors. For example, the pattern seen on the zoomed part of the Figure 4.2 was found to be due to modulation of the cavity output coupling induced by the standing waves between the detector and the cavity output mirror. The pattern has a period of $\sim 22$ points, which is consistent with a separation between the detector and a coupling mirror of $\sim 45.5 \mathrm{~cm}$. The broader structure is possibly due to the reflections from the chamber walls. The origin of these baseline 
variations, especially whether they come from the inside (e.g. wall reflections) or outside (e.g. detector reflections) of the cavity dictates how this pattern changes when a gas is added to the cavity.

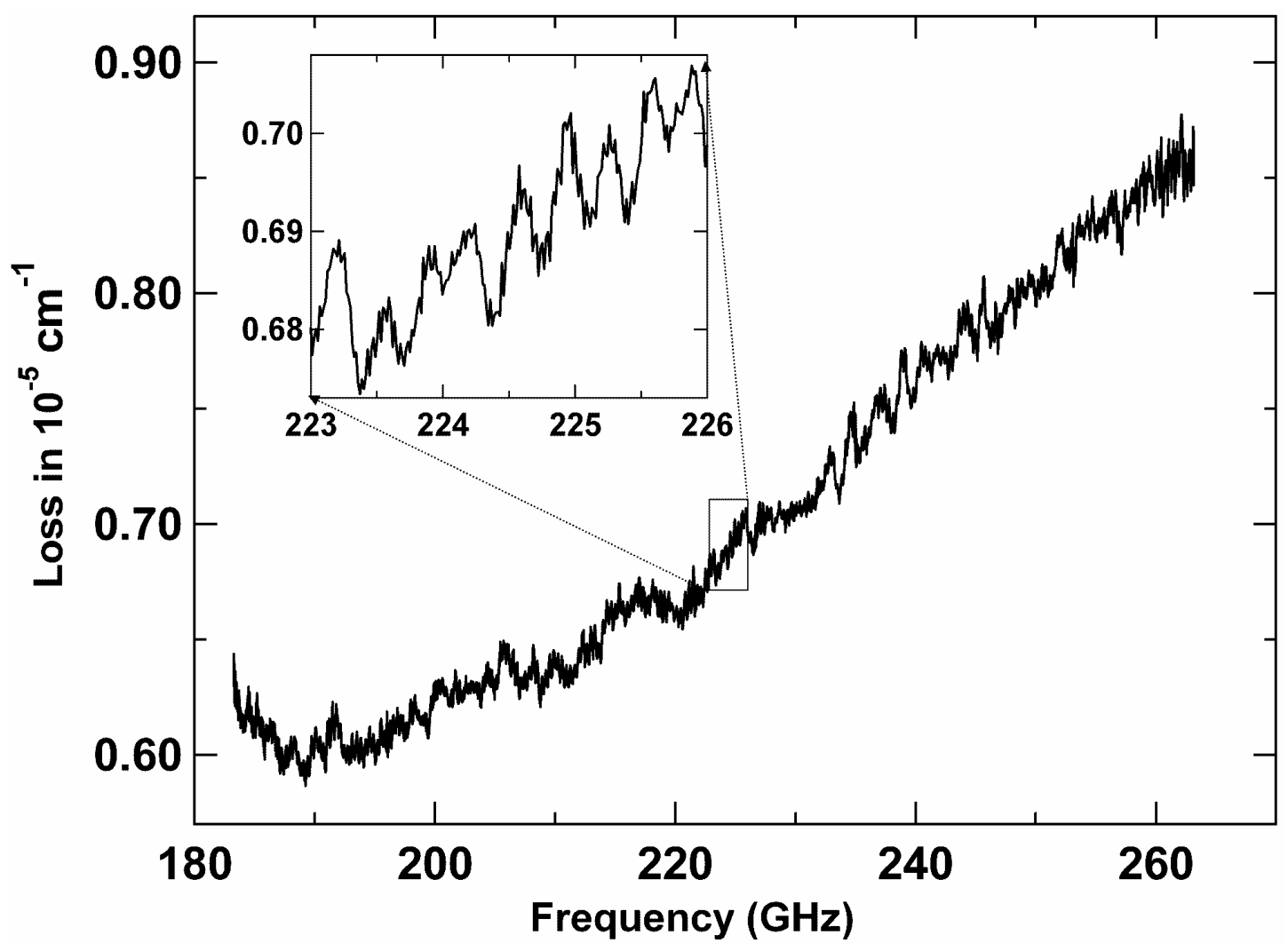

Figure 4.2: Baseline structure of the empty resonator loss. 50 scans averaged, Fourier Transform processed. The structure on the zoomed part of the graph is due to the detector reflections.

\subsection{SYSTEMATIC EFFECTS}

Even with the small integration times used in this experiment, individual resonator loss measurements can be made, by integration of 100 three second scans, with uncertainties on the order of $10^{-8} \mathrm{~cm}^{-1}$ for each of the 6000 resonance frequencies. Further averaging of measurements made at different frequencies is 
possible because of the slow variation of measured effects with frequency. For the gas absorption measurements to be as accurate as the ones of the resonator loss, all the other resonator losses would have to stay the same with and without the gas. In reality, none of the losses stay exactly the same, although most changes are either negligible or can be predicted and included in the subtraction model. Still, the systematic effects set the current limits on absorption measurement accuracy.

\subsubsection{Index of refraction effects, background pattern, Ar measurements}

Mirror reflection is the only resonator loss that does not change as a result of changes in the index of refraction introduced when the resonator is filled with a gas. Increasing the index of refraction shrinks the diameter of the FP resonator modes according to equation (3.7), thus reducing the diffraction loss, but increasing the output coupling loss. It also decreases the input coupling loss. Simple estimates show that for our instrument most of these changes are small compared with the gas absorption loss even in the case of the dry gas absorptions. We will discuss these changes in more detail below.

Using equations (3.6) and (3.11) and assuming that the changes are small, we can calculate the diffraction loss change to be

$$
\Delta \delta_{d}=-\delta_{d} \frac{2 R^{2}}{\omega^{2}} \frac{d n}{n}=-\exp \left[-2\left(\frac{R}{\omega}\right)^{2}\right] \frac{2 R^{2}}{\omega^{2}} \frac{d n}{n} .
$$

For $R=1.8 \omega$, corresponding to the highest $(\sim 0.15 \%)$ diffraction loss at low frequency, and for the 3 atm of nitrogen $\left(\frac{d n}{n} \approx 10^{-3}\right)$, the diffraction loss change is

$$
\Delta \delta_{d} \approx-110^{-5}=-0.001 \%
$$


or $\sim 2 \%$ of the nitrogen absorption for the same conditions. This effect can be neglected for oxygen and moist air absorption measurements but it can slightly affect the nitrogen and the dry air measurements below $175 \mathrm{GHz}$.

From equations (3.6) and (3.24) we can get an expression for the change of the output coupling loss:

$$
\Delta \delta_{c o}=\delta_{c o} \frac{d n}{n}
$$

Because $\Delta \delta_{c o} \leq 0.1 \%$ for all frequencies,

$$
\Delta \delta_{c o} \leq 10^{-6}
$$

and can be neglected.

The change in the input coupling coefficient $\Delta \delta_{c i}$ increases as a frequency squared, just like all the continua effects. For 0.5 mil Mylar beam splitter with index of refraction 1.83 , the change in the input coupling coefficient from introducing 3 atmospheres of nitrogen into the chamber at room temperature can be calculated by using equation (3.18) and is equal to

$$
\Delta \delta_{c i} \approx 1.110^{-8} v^{2}
$$

where $v$ is the radiation frequency in GHz. This is about $1 \%$ of the absorption of nitrogen at the same pressure. Because $\Delta \delta_{c i}$ has the same frequency dependence as the nitrogen absorption, pressure dependence has to be used to separate the two effects. Nitrogen absorption is proportional to the pressure squared while $d n$ and $\Delta \delta_{c i}$ increase linearly with pressure. Although this systematic effect is negligible in moist air absorption measurements, it was shown to decrease the power coefficient in the absorption pressure dependence of dry air and had to be taken into account in our data analysis. 
Another effect of the index of refraction change is the shift in the Fabry Perot resonance frequencies. When the resonator is filled with the gas of index of refraction $n$, the resonance frequencies are given by the modified equation (3.40)

$$
v_{k}=k \frac{c}{2 \operatorname{Ln}} .
$$

As was described above, five water lines are used to determine the frequency of each resonance in the empty cavity to better than $1 \mathrm{MHz}$ precision. The small scale structure of the BWO frequency-voltage characteristic can be used to find the resonance frequencies of the filled cavity. Because the FSR of the filled resonator is smaller than that of the empty resonator, there are more filled cavity resonances in the same frequency range. More specifically,

$$
\frac{k_{\text {filled }}-k_{\text {empty }}}{k_{\text {empty }}}=\frac{n_{\text {filled }}-n_{\text {empty }}}{n_{\text {empty }}}=n_{\text {filled }}-1
$$

For about 6000 resonances in the full scan range of $170-260 \mathrm{GHz}$ and 3 atm of nitrogen, there are about 5 more resonances in the filled cavity than in the empty cavity, which is displayed as a shift in the small scale structure. This shift will be discussed in detail in section 4.3. It provides a direct way of calibrating the filled cavity frequency and measuring the gas index of refraction by using equations (4.6) and (4.7). It also presents a choice of the subtraction strategy. We can either match each filled cavity resonance with the closest in frequency empty cavity resonance, or we can match one such couple and then subtract fringes with the same number. The second method is less computationally involved and is used for all our data processing. The systematic error introduced by subtracting the losses at different frequencies can be estimated from the empty cavity loss and the calculated frequency shift and was shown to be negligible. 
To summarize, the change in the index of refraction changes all the radiator losses but the mirror reflection loss. It also changes the resonance frequencies. The input coupling and the diffraction coupling losses decrease with the addition of the gas, while the output coupling loss increases. All of these changes are small even compared with the dry air or nitrogen absorption.

A direct experimental check is possible to confirm these theoretical predictions. Argon and nitrogen have similar indices of refraction (for $1 \mathrm{~atm}, n-1$ is $277 \times 10^{-6}$ for argon and $294 \times 10^{-6}$ for nitrogen). ${ }^{66}$ However, argon has no absorption in the microwave. As shown in Figure 4.3, adding $3 \mathrm{~atm}$ of argon to the chamber, although significantly changing the background pattern and the measurement of the loss at any particular frequency, does not change the system loss, averaged over the frequency of the standing wave patterns. This also both illustrates the significance of the changes in the background loss contribution to the error in the gas absorption measurements and provides a way to significantly reduce this contribution. Instead of the empty cavity loss, the loss of the resonator with the appropriate amount of $\mathrm{Ar}$ can be used as a baseline to be subtracted from the absorbing gas filled resonator loss. This practically doubles the time of the experiment but makes a noticeable improvement in the absorption measurement accuracy. 


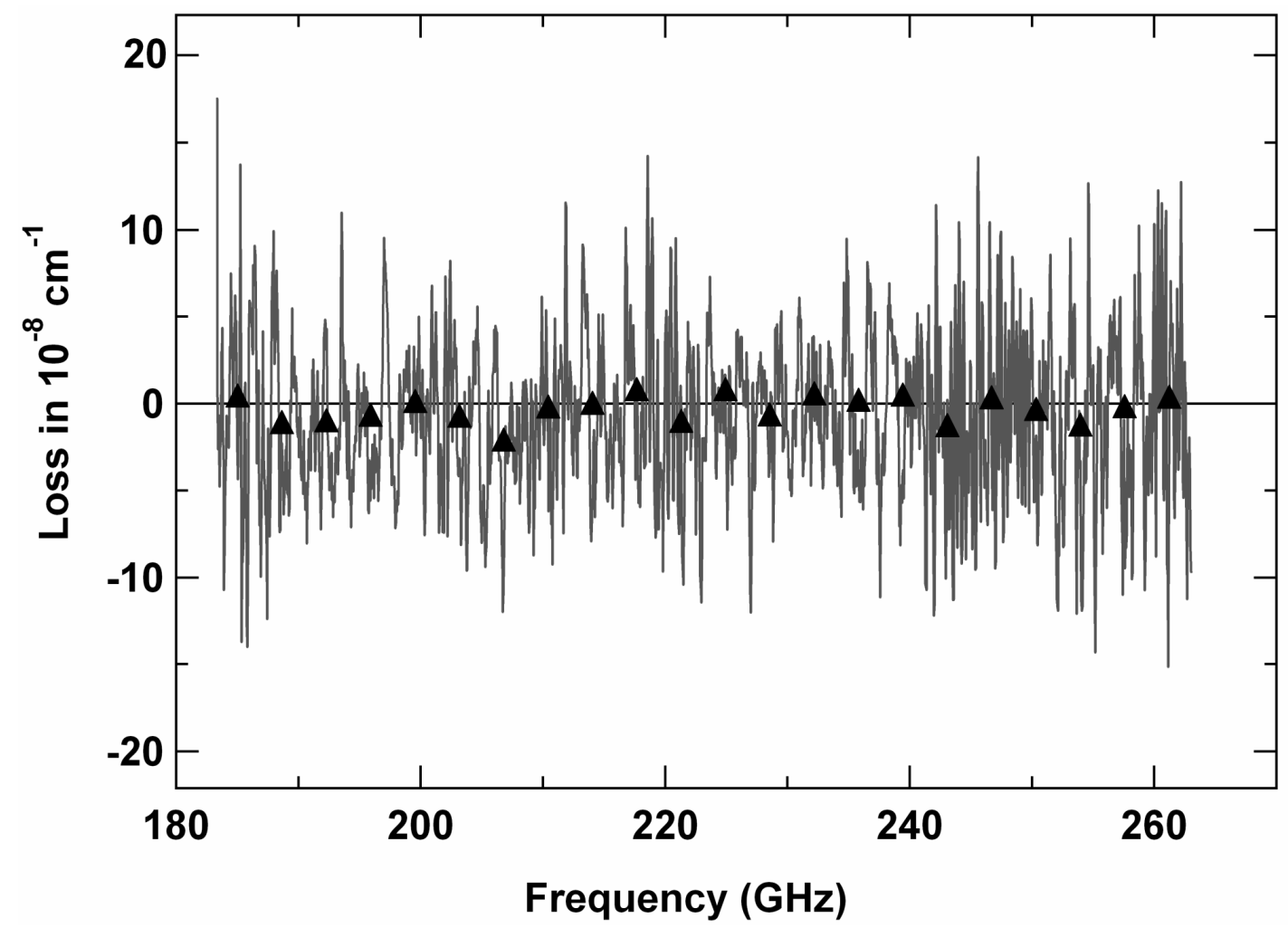

Figure 4.3: FT processed loss of the resonator filled with 2280 Torr ( $3 \mathrm{~atm}$ ) of Ar, after subtracting the empty resonator loss. No averaging (grey) and 250 points average ( $\mathbf{\Lambda})$.

\subsubsection{Temperature effects}

Care is taken to minimize the temperature changes. The thermally insulated enclosure combined with the automated temperature control keeps the average temperature of the chamber within $\pm 0.5^{\circ} \mathrm{C}$ during the experimental run. Effects of thermal expansion and contraction during the run on the absorption measurements are negligible, but a one degree change in mirrors temperature will lead to a change in mirror reflectivity of about $1 \%$ of the 3 atm of nitrogen absorption. Indeed, the temperature coefficient of resistance for copper is $\sim 0.4 \% /{ }^{\circ} C$. This leads to $\sim 0.2 \% /{ }^{\circ} \mathrm{C}$ change in the mirror reflection loss according to equation (3.2). Therefore for the copper mirror with a reflection loss $\delta_{r} \approx 0.3 \%$, a one degree change in the mirror temperature would cause a $\sim 0.0006 \%$ change in the 
reflection loss, equivalent to about $1 \%$ absorption loss of $3 \mathrm{~atm}$ of nitrogen. Although small, this effect was included in our data analysis when necessary, based on the available temperature data.

\subsubsection{Moist air specific effects}

Because we measure the gas phase water amount from the water line profile, we do not have to worry about the water partial pressure changing due to the wall condensation. The water partial pressure is simply another parameter in the absorption model. Another, more difficult to account for effect, is the change in mirror reflectivity due to water adsorbing on the FP mirrors surfaces. This effect increases at lower temperatures, becoming the dominating factor in the absorption measurement accuracy below the water freezing point. The ability of our system to make fast broad frequency measurements is invaluable in separating this effect from the actual gas absorption based on the analysis of the absorption data for different water and dry air pressures.

As an example, let us consider measurements of the moist continuum we made at $-23{ }^{\circ} \mathrm{C}$. For low water partial pressures, moist continuum absorption is expected to be proportional to the product of water and dry air pressures. ${ }^{28}$ Figure 4.4 shows how the measured resonator loss varied as a function of this product. Each point on the graph presents a coefficient obtained from fitting the data to a quadratic frequency dependence after subtracting the water line contribution. The linear part of this curve is (to within experimental uncertainty) independent of the value at which the gas is pumped from the chamber over a wide range of pumping speeds. However, the curve near zero pressure can vary as the pumping speed and time to reach 
equilibrium become comparable. This is consistent with water leaving the mirrors faster at lower pressures, with most of mirror reflectivity changes happening at the low pressure part of the graph. A more detailed investigation of this effect is presented later in this chapter in the Moist Air results section.

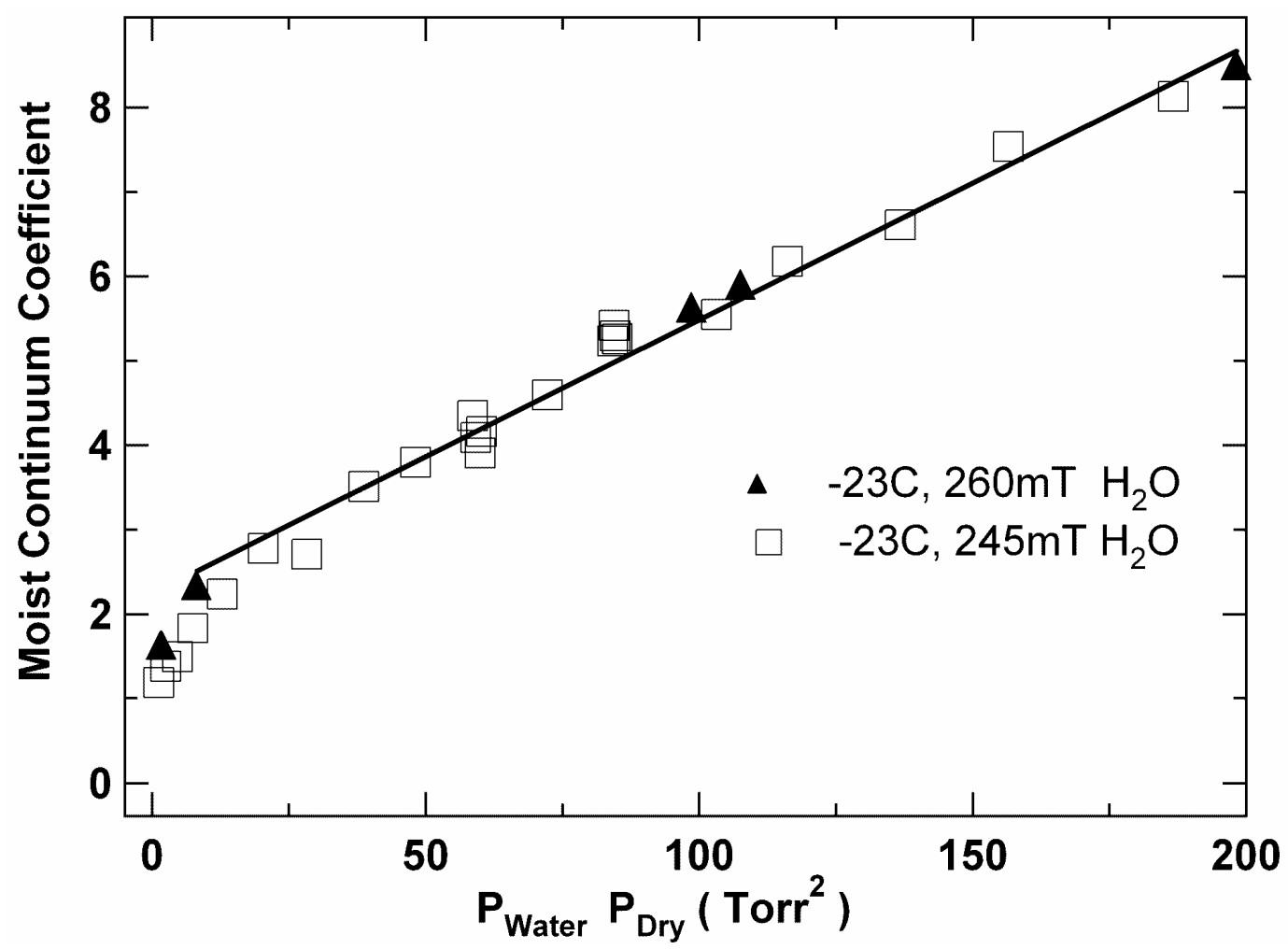

Figure 4.4: Moist continuum absorption dependence on the product of water and dry air pressures at $-23^{\circ} \mathrm{C}$. Two days of data are shown with the same temperature and close starting water pressures (at 1 atmosphere of the total moist air pressure).

\subsubsection{Detection bandwidth effects}

It is important that the radiation decay times are determined correctly since any inaccuracy in the exponential decay time measurement will be passed directly to the measured absorption. For a particular detection bandwidth, there is a maximum system loss for which no consideration of the signal distortion by the detector and the amplifier is needed. Greater losses will lead to the radiation intensity decaying too 
fast for the measurement electronics to follow and non-exponential output signal decays. Although it is possible to explicitly include the detection electronics response in the fitting routine,${ }^{67}$ knowing how high in absorption one can go without having to do so is helpful.

Based on the measured response of our detector-amplifier pair and mathematical modeling of the changes to the exponentially decaying input signal due to the limited detection bandwidth, we have predicted that for the decay times longer than $1 \mu$ s (corresponding to a total resonator loss per pass less than $3.3 \%$ ) no additional corrections to the measured ringdown times are required. This prediction is confirmed by the investigation of the water absorption line shapes close to the line center. Insufficient detector bandwidth would distort the line shape by reducing the effective measured absorption at the center of the line, where the ringdown decay times are shortest. No systematic distortion of this kind was detected for any of the moist air measurements. A good visual demonstration is given in Figure 4.5 for 2 Torr partial pressure of water. 


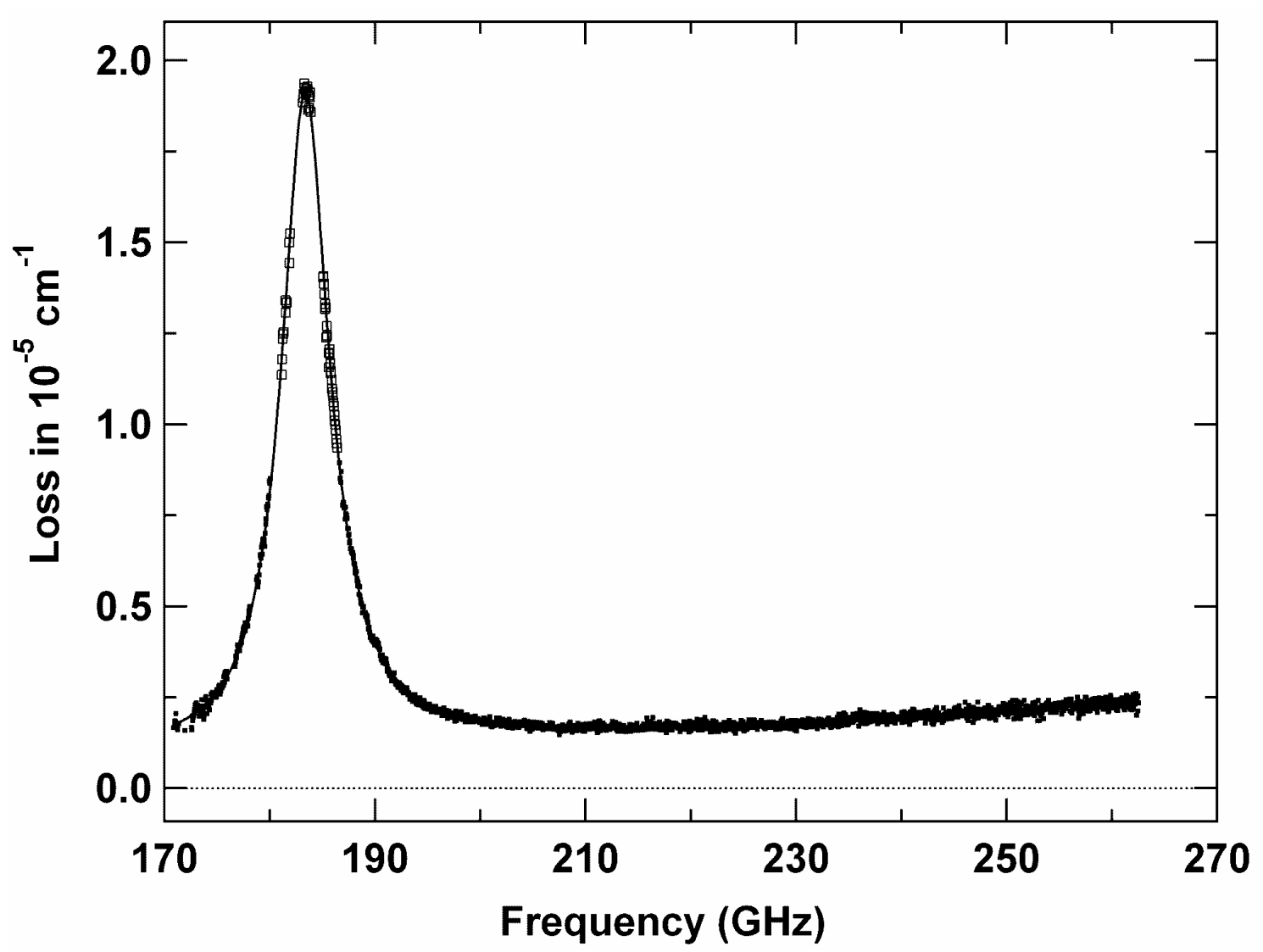

Figure 4.5: Moist air absorption with 2 Torr partial water pressure. The solid line is a fit to the Van Vleck-Weisskopf profile and a quadratic in frequency continuum absorption term. Only frequencies with absorption below one half of the peak absorption ( $\square)$ are used in the fit. The rest of the points ( $\square$ ) are presented on the same graph, showing no signs of the line profile distortion related to insufficient detecting bandwidth. 


\subsection{FILLED RESONATOR FREQUENCY CALIBRATION, INDEX OF REFRACTION MEASUREMENTS}

It was described above how the frequency of all of the empty cavity resonances is calibrated to better than $1 \mathrm{MHz}$ accuracy. As we add gas to the chamber, the free spectral range of the resonator changes, thus changing the resonance frequencies. If the index of refraction $n$ is known, the FSR of the filled resonator can be calculated and the frequency of each resonance can be calculated as

$$
v_{\text {filled }}(k)=v_{\text {filled }}(0)+k \frac{F S R_{\text {empty }}}{n},
$$

provided the frequency of at least one filled cavity resonance is known.

Experimentally the best way to calibrate the frequency of the filled resonator is to use the stability of the BWO small scale structure. ${ }^{62}$ From scan to scan the pattern of the voltage (or time) separation between the FP resonances stays remarkably constant. By comparison of these small scale patterns for the empty and filled cavity, we can find the corresponding frequencies for the filled cavity resonances to a fraction of the free spectral range of the resonator, or to a few $\mathrm{MHz}$, as described below. 


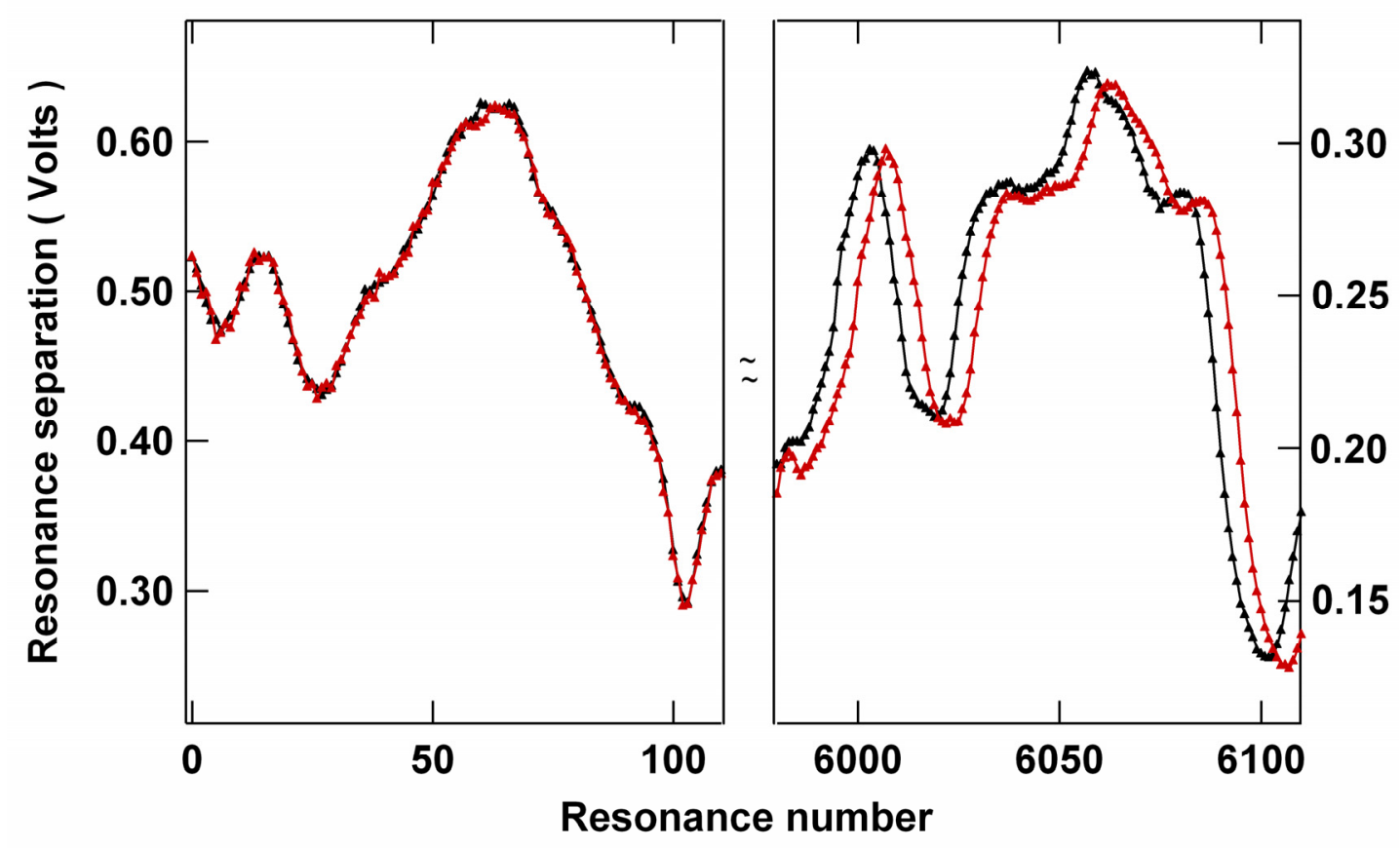

Figure 4.6: Typical small scale structure of the frequency-voltage dependence of a BWO. Resonance separation as a function of the resonance number for frequency scans of an empty resonator (black) and a resonator filled with $3 \mathrm{~atm}$ of nitrogen (red).

Figure 4.6 shows a comparison of the BWO small scale structure for the empty and filled resonator for two scan position; one at the start, another close to the end of the scan. The pattern of this structure stays constant with frequency, while the shift that can clearly be seen at the end of the scan is a direct result of the difference in the index of refraction between the scans. This shift increases linearly with the Fabry-Perot fringe number as shown in Figure 4.7 and the slope of this linear function is equal to $n-1$. Indeed, if $k_{1}$ and $k_{2}$ are the resonance numbers of the empty and filled resonator, respectively, and $n$ is the index of refraction, than the constant frequency requirement leads to

$$
\frac{k_{1}}{1}=\frac{k_{2}}{n}
$$




$$
n-1=\frac{k_{2}-k_{1}}{k_{1}}=\frac{d k}{k} \text {. }
$$

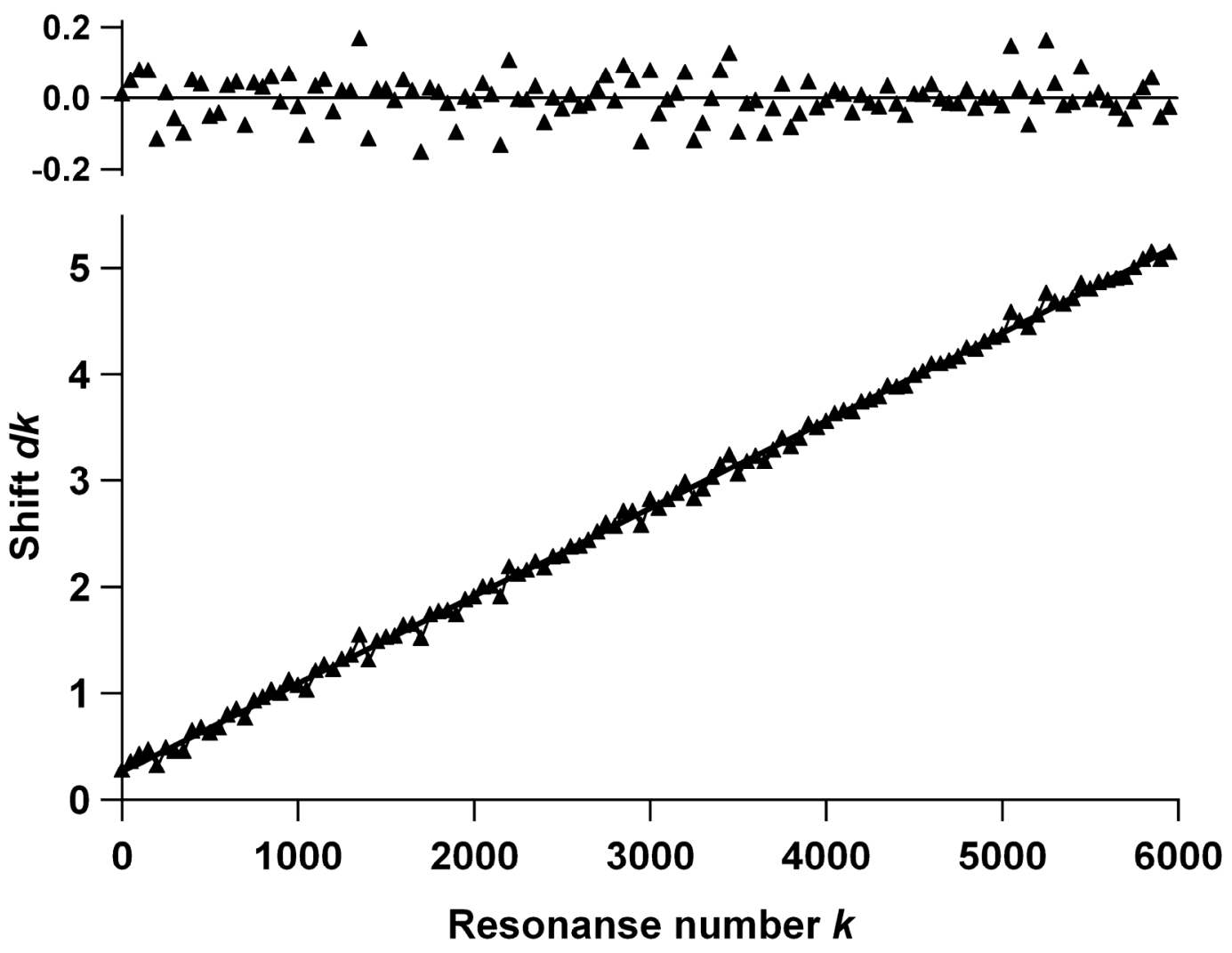

Figure 4.7: The BWO small scale structure shift $d k$ of the resonator filled with 3 atm of nitrogen with respect to an empty resonator as a function of the Fabry-Perot resonance number $k$. The residual of the fit to a linear function is presented above.

From the linear fit of the shift as a function of the resonance number, the shift of the first resonance and the index of refraction can be found, and the filled cavity resonance frequencies can be calculated according to equation (4.8). As predicted theoretically, index of refraction, calculated as described above, increased linearly with pressure for all our measurements. Example of this dependence for room temperature measurements of nitrogen, oxygen and dry air is shown in Figure 4.8. 


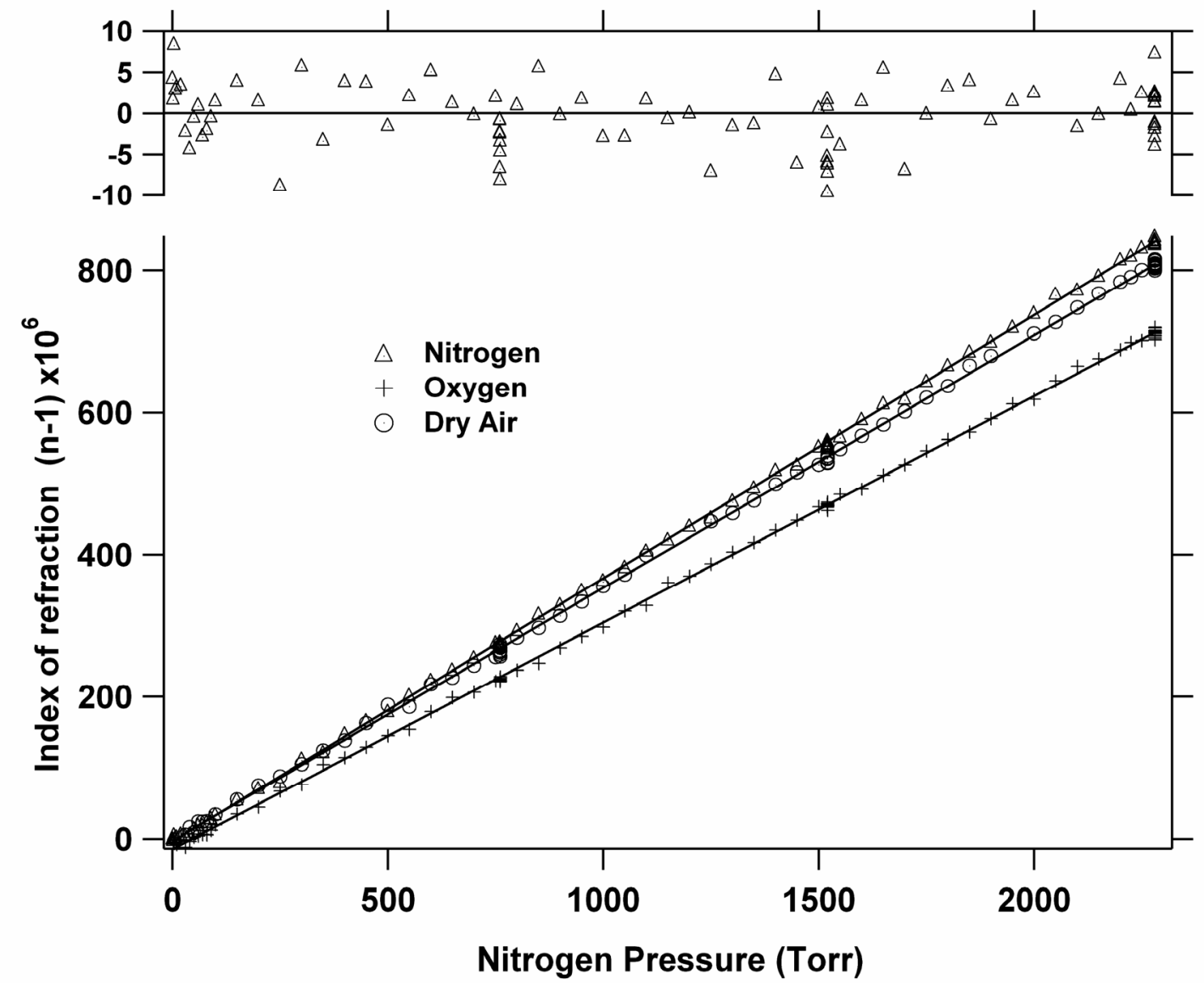

Figure 4.8: The room temperature nitrogen, oxygen, and dry air indices of refraction pressure dependence. The residual of the nitrogen data fit to a linear function is presented above.

The index of refraction measurement results for nitrogen, oxygen and dry air for various temperatures are presented in the Table 4.1 and Figure 4.9. For each temperature, the index of refraction pressure dependence was fitted with a linear function as

$$
(n-1) 10^{6}=C(T) \frac{P(\text { Torr })}{760} .
$$


The temperature coefficients $C(T)$ were later fitted with a power law to check the temperature dependence as

$$
C(T)=C_{0}\left(\frac{T}{300 K}\right)^{n_{T}}
$$

\begin{tabular}{|c|c|c|c|c|c|}
\hline \multicolumn{2}{|c|}{ Nitrogen } & \multicolumn{2}{|c|}{ Oxygen } & \multicolumn{2}{|c|}{ Dry Air } \\
\hline Temperature & $C(T)$ & Temperature & $C(T)$ & Temperature & $C(T)$ \\
\hline 251.6 & 314.0 & 252.0 & 280.4 & 230.5 & 330.2 \\
\hline 261.9 & 302.7 & 272.8 & 257.3 & 241.8 & 311.5 \\
\hline 273.0 & 289.4 & 296.7 & 238.9 & 252.1 & 302.7 \\
\hline 296.9 & 269.3 & 302.9 & 233.1 & 261.4 & 295.5 \\
\hline 297.5 & 271.0 & 323.6 & 218.2 & 275.7 & 276.1 \\
\hline 297.4 & 267.9 & 340.9 & 207.7 & 297.2 & 262.7 \\
\hline 300.6 & 262.8 & & & 298.7 & 256.6 \\
\hline 305.9 & 261.2 & & & 313.4 & 249.2 \\
\hline 313.8 & 254.3 & & & 322.1 & 236.4 \\
\hline 323.1 & 245.1 & & & 331.5 & 229.1 \\
\hline \multirow[t]{2}{*}{332.6} & 239.1 & & & 341.0 & 225.5 \\
\hline & & & & 350.1 & 220.7 \\
\hline \multicolumn{2}{|c|}{$C_{0}{ }^{\text {Nitr }}=265.3(5)$} & \multicolumn{2}{|c|}{$C_{0}{ }^{x}=235.4(4)$} & \multicolumn{2}{|c|}{$C_{0}^{D r y}=256.0(9)$} \\
\hline \multicolumn{2}{|c|}{$n_{T}=-0.97(2)$} & \multicolumn{2}{|c|}{$n_{T}=-0.99(1)$} & \multicolumn{2}{|c|}{$n_{T}=-0.96(2)$} \\
\hline
\end{tabular}

Table 4.1: Nitrogen, oxygen, and dry air index of refraction measurement results. 


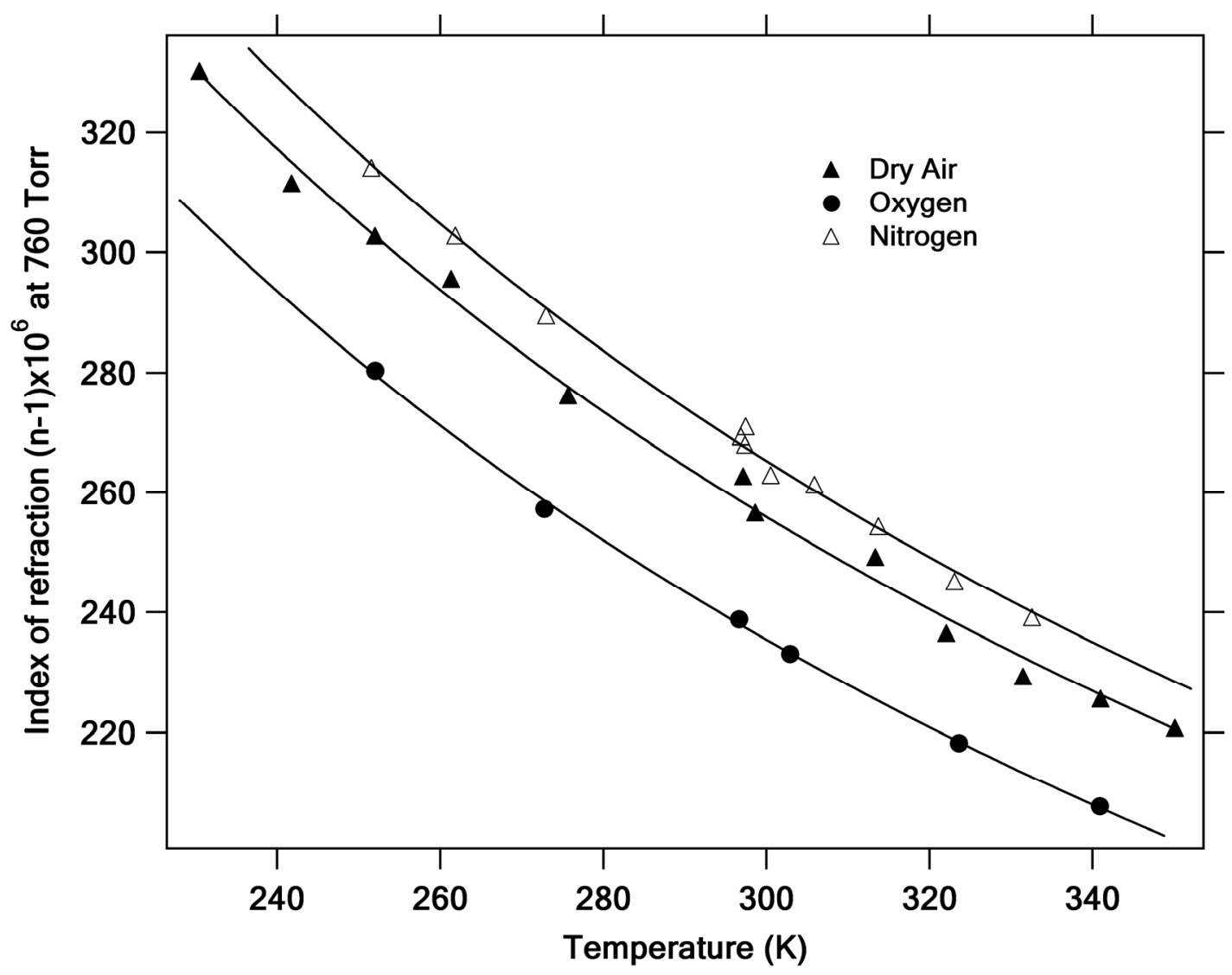

Figure 4.9: Nitrogen, oxygen, and dry air index of refraction temperature dependence. Solid lines are fits to the power law temperature dependence.

The statistical uncertainty of the coefficients $C(T)$, obtained from the linear fit of the index of refraction as a function of temperature, was always below $0.3 \%$, so the actual error of each measurement is better represented by the scatter of the results from the power law in Figure 4.9. This error is estimated to be about $2 \%$.

The chamber temperature and pressure changes between the empty and filled cavity had to be taken into account for all the $n$ calculations. This has to be done because the change in the mirror separation $L$ due to a one degree change in the chamber temperature shifts the small scale BWO structure just as much as the gas with $n-1=15.6 \times 10^{-6}$. Just as the L change due to 3 atm of gas shifts the frequency as the gas with $n-1=23.4 \times 10^{-6}$. This can be seen from equations (3.58), (3.59), and 
the fact that for the small changes in $n$ and $L$ the frequency constant requirement is equivalent to

$$
\frac{d n}{n}=-\frac{d L}{L}
$$

The measured temperature dependence is consistent with the fact that index of refraction is proportional to the gas density, which scales inversely with temperature. The results are also in reasonable agreement with measurements by Newell et al., ${ }^{66}$ 267.51(6), 243.06(5) and 262.34(5) for the nitrogen, oxygen and the dry air, respectively, reasserting the chosen strategy for the filled resonator frequency calibration. The probable reason that our results are lower by $1 \%$ to $3 \%$ than the literature is a poor estimation accuracy of the temperature and pressure effects described above. The simplest model was chosen and no additional testing was performed to better understand these effects since this accuracy is more than adequate for the filled resonator frequency calibration.

As can be seen from Figure 4.7, by using the strategy described above, we can find the frequency of each filled cavity resonance with better than $0.2 \mathrm{FSR}$, or $\sim 3 \mathrm{MHz}$. In general, this type of precision is not required because the measured absorption changes much slower with frequency. In most cases we match the frequencies of one pair of resonances (empty and filled chamber) and subtract absorptions of the resonances with the same indexing number. With this approach we subtract absorptions at different (by up to $50 \mathrm{MHz}$ ) frequencies but corresponding to the same indexing mode numbers of the Fabry Perot resonator. The absorption measurement error introduced by the frequency difference is negligible, but the subtraction is simple and straightforward, at the same time minimizing the scatter due 
to the change in the resonance number. The ability to calibrate the filled resonator frequency is critical for the measurement of the $183 \mathrm{GHz}$ water line pressure shift and pressure broadening parameters, discussed below.

\subsection{GAS ABSORPTION MEASUREMENTS}

\subsubsection{Nitrogen absorption}

A good starting point for evaluation of the system performance is measurement of the nitrogen absorption. Nitrogen, the most abundant atmospheric gas at $78 \%$ by volume, is a homonuclear diatomic molecule and has no allowed dipole rotational transitions. As described in Chapter 2, at long wavelength nitrogen's dominant absorption mechanism is collision-induced absorption (CIA) that peaks in the $\mathrm{THz}$ region and slowly varies with frequency. The CIA increases as the nitrogen pressure squared and is very small - less than $1 \%$ in $1 \mathrm{~km}$ at room temperature and atmospheric pressure for our frequency range. Nitrogen therefore provides a good test of the system sensitivity and an excellent means to study the systematic effects.

Prepurified nitrogen (99.998\% purity) from Praxair was used for all our nitrogen measurements. Absorption of $3 \mathrm{~atm}$ of room temperature nitrogen, calculated by subtracting the loss of the empty cavity from the loss of the cavity filled with the gas, is shown in Figure 4.10. The 66 point averages (frequency intervals of $\sim 1 \mathrm{GHz}$ ) of the same data are presented in the figure along with the $v^{2}$ fit. The statistical uncertainty of the fit coefficient is below $1 \%$. 


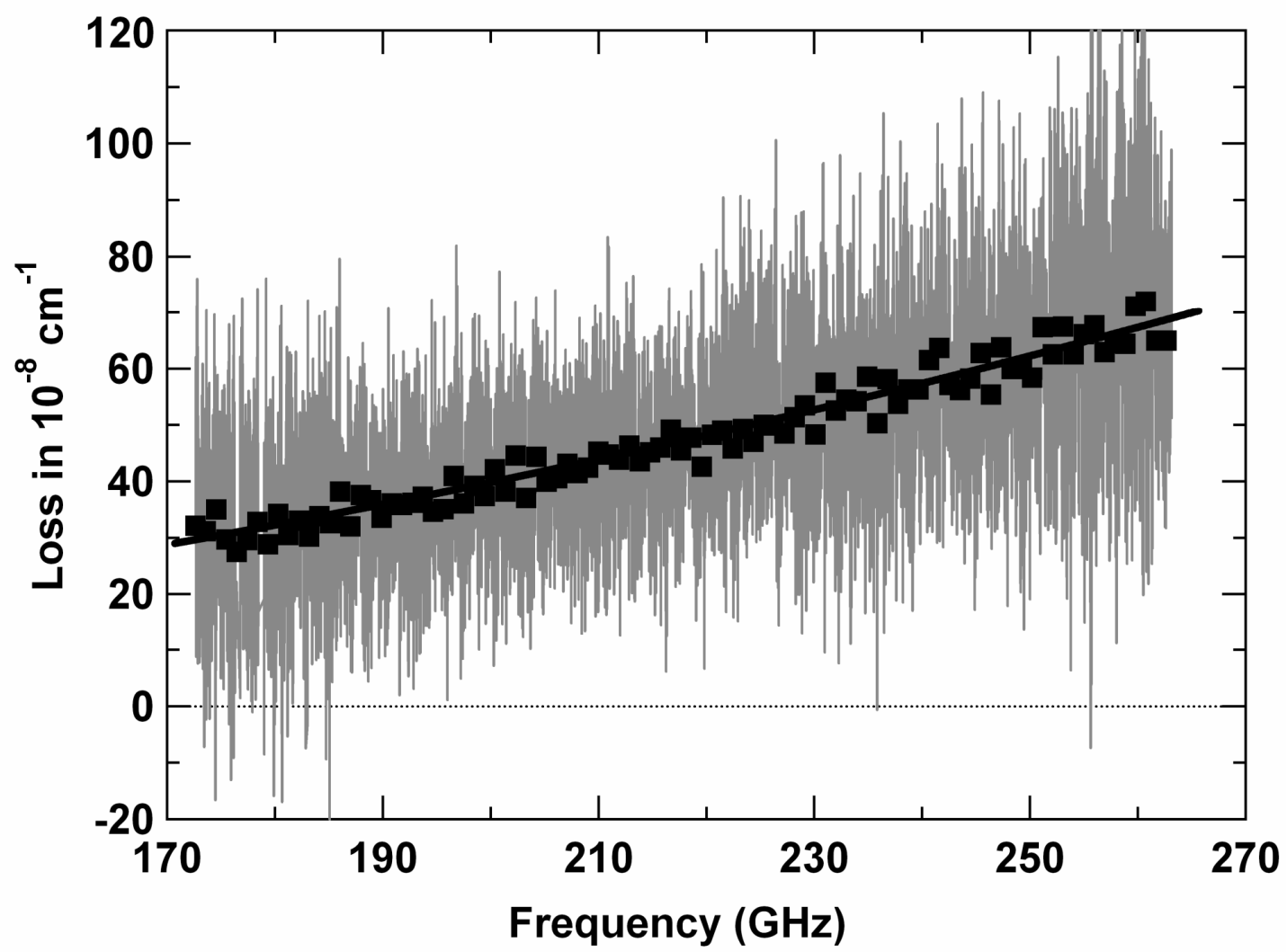

Figure 4.10: Absorption of $3 \mathrm{~atm}$ of pure nitrogen as a function of frequency. Single scan, no averaging (grey), 66 point averages (black markers) and the $v^{2}$ fit (solid black line).

Because of the changes in the index of refraction, the baseline structure illustrated in Figure 4.2 is different for the gas-filled and empty cavity. This introduces systematic error in the form of the periodic pattern in the absorption, as seen in Figure 4.11, which shows the average of 50 scans of the empty cavity, subtracted from the average of 50 scans of the filled cavity. 


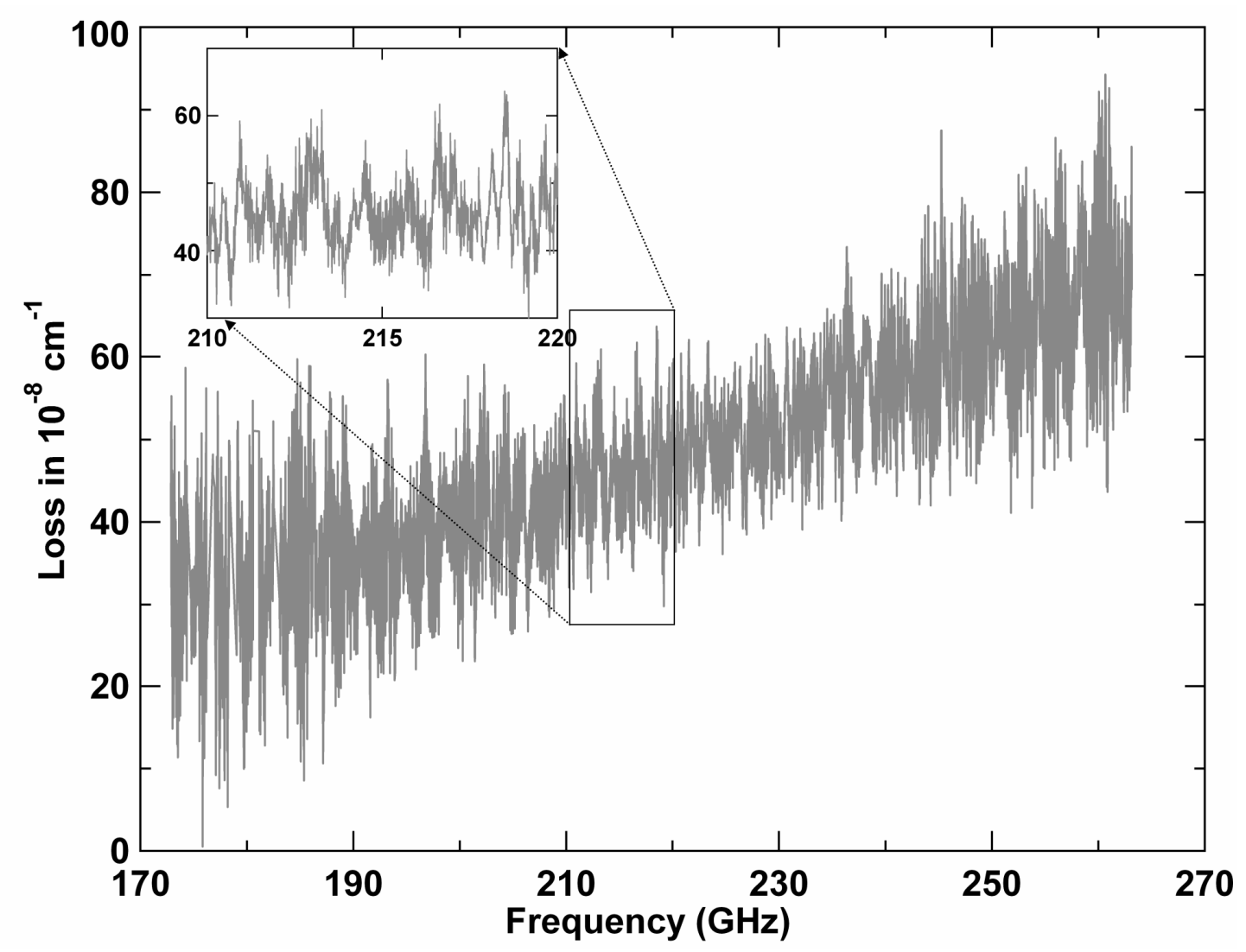

Figure 4.11: 50 scan-averaged absorption of 3 atm of pure nitrogen as a function of frequency. Systematic effect in the form of the standing waves of varying periods can be seen after the averaging.

Nitrogen absorption was measured at a number of temperatures from -40 to $+60{ }^{0} \mathrm{C}$. For each temperature, measurements at about 50 different pressures up to 3 atm were made with $\sim 6000$ ringdown times measured at each pressure for frequencies in the 170-260 GHz range. For all the pressures and temperatures the nitrogen absorption varied as frequency squared within the experimental uncertainty. A simplified sequential analysis is possible if for each pressure and temperature the single frequency squared fit coefficient is calculated from the 6000 individual frequency measurements. Taking this approach further, Figure 4.12 shows the results of a sequential fit of the nitrogen absorption pressure dependence, with each point on 
the graph derived from an earlier fit of absorption as a function of frequency. As expected, the nitrogen absorption pressure dependence was well described by a quadratic function for all the measured temperatures.

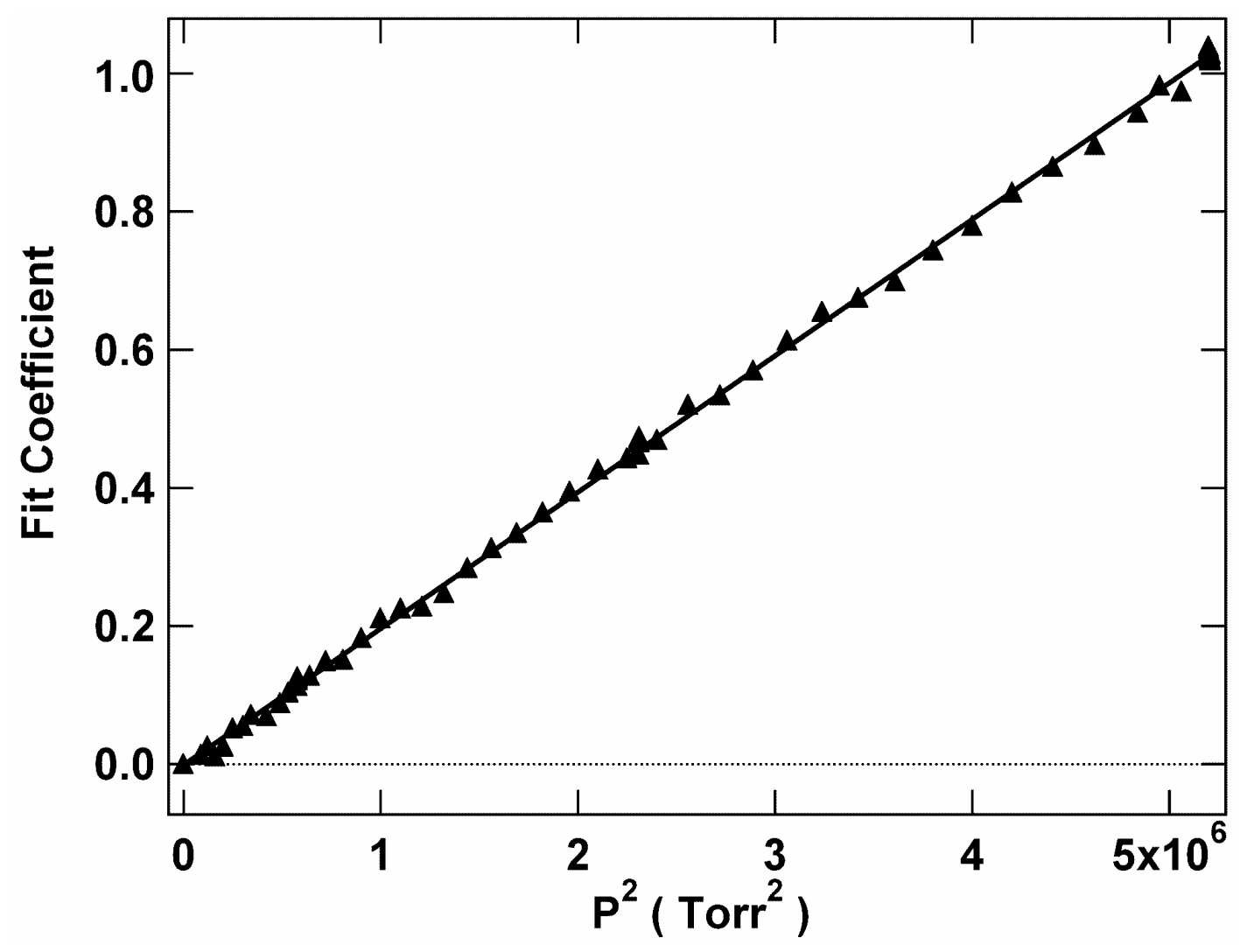

Figure 4.12: The magnitude of the room temperature nitrogen absorption $v^{2}$ fit coefficient as a function of the nitrogen pressure squared. The solid line is a linear fit of the data.

Now, assuming a quadratic dependence on pressure and frequency and a power law dependence on temperature, nitrogen absorption in the 170-260 GHz range can be described by

$$
\alpha_{\text {Nitr }}=C(T) P^{2} v^{2}=C_{0} P^{2} v^{2} \theta^{n},
$$

with

$$
\theta=\frac{T}{300 K} .
$$


The spectroscopic uncertainty of $C(T)$, obtained from the fits of the data as a function of pressure and frequency, was always less than $0.3 \%$ and the accuracy of the measured $C(T)$ is limited by the uncertainties in pressure and temperature measurements. We estimate our temperature uncertainty at $\pm 1{ }^{0} \mathrm{C}$ and the relative pressure uncertainty at $0.5 \%$. The uncertainty in $C(T)$ can than be calculated as

$$
\frac{\Delta C(T)}{C(T)}=2 \frac{\Delta P}{P}+n \frac{\Delta T}{T}=0.01+\frac{3.3}{T}
$$

where 3.3 is used as a temperature exponent, obtained from the non-weighted fit. Uncertainties, calculated with 4.16 are given in Table 4.2 and are used in the weighted fit, shown in Figure 4.13. 


\begin{tabular}{|c|c|}
\hline Temperature $(\mathrm{K})$ & $\mathrm{C}(\mathrm{T})\left(10^{-18} \mathrm{~cm}^{-1} /\left(\right.\right.$ Torr $\left.\left.^{2} \mathrm{GHz}^{2}\right)\right)$ \\
\hline 323.1 & $1.498(30)$ \\
\hline 313.8 & $1.602(33)$ \\
\hline 305.9 & $1.721(36)$ \\
\hline 300.6 & $1.891(40)$ \\
\hline 297.5 & $1.963(41)$ \\
\hline 297.4 & $1.915(40)$ \\
\hline 296.9 & $1.929(41)$ \\
\hline 273.0 & $2.564(57)$ \\
\hline 261.9 & $2.890(65)$ \\
\hline 251.6 & $3.417(79)$ \\
\hline 228.8 & $4.52(11)$ \\
\hline & $C_{0}=1.876(13)$ \\
\hline & $\mathrm{n}=-3.28(7)$ \\
\hline
\end{tabular}

Table 4.2: Measured nitrogen absorption coefficients $C(T)$. 

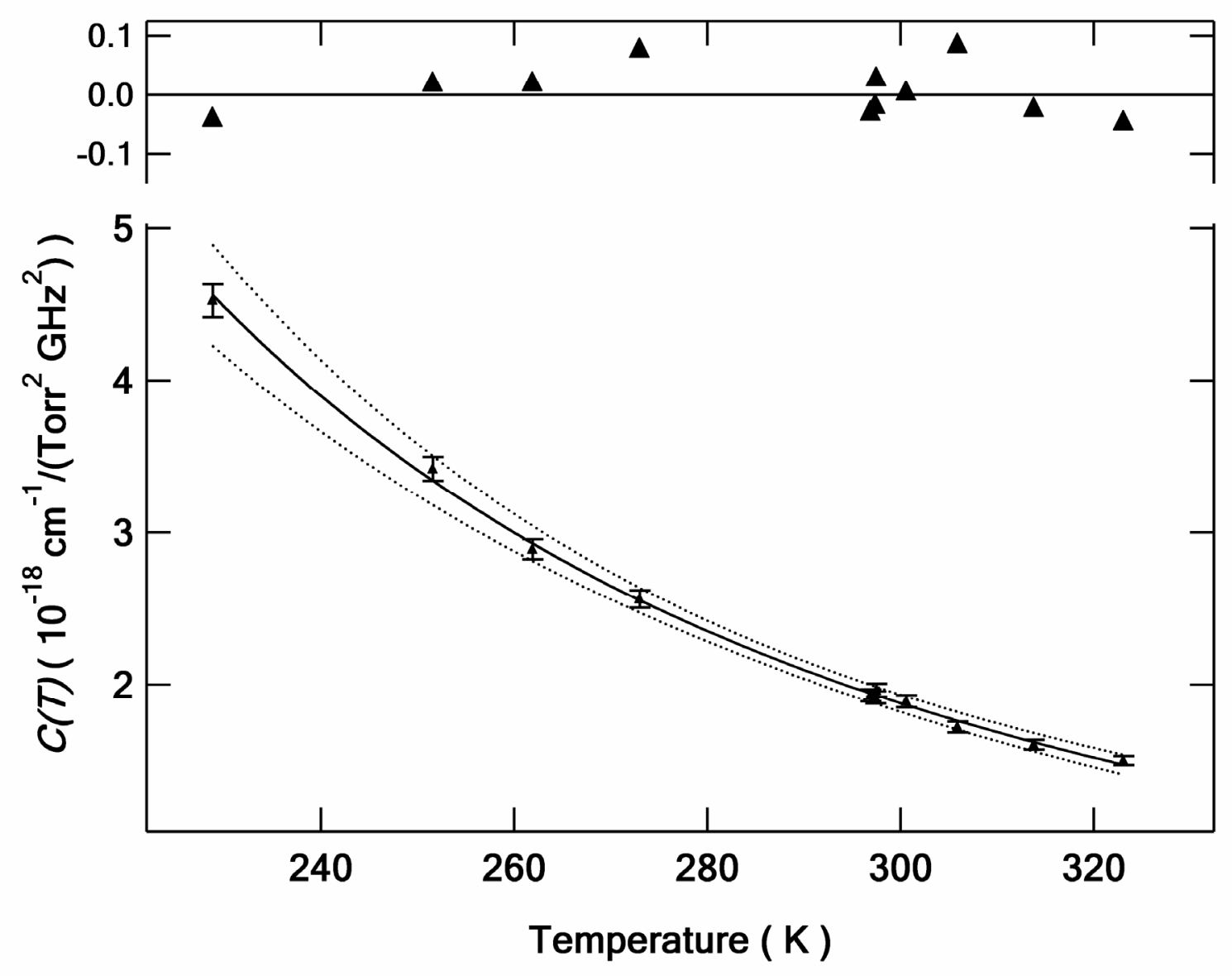

Figure 4.13: Nitrogen absorption temperature dependence. Solid line is a result of the temperature power law fit. Dotted lines are the $3 \sigma$ confidence bands. The residual of the fit is shown at the top of the figure on an expanded scale.

It is important to realize that the uncertainty of the fit coefficient $C_{0}$ is not a direct indication of the uncertainty in the nitrogen absorption prediction for certain gas parameters. To demonstrate this point, if the nitrogen absorption is fitted with a modified equation (4.14) as

$$
\alpha_{\text {Nitr }}=C(T) P^{2} v^{2}=C_{1} P^{2} v^{2} T^{n}
$$

the fit results are

$$
C_{1}=2.4(9) 10^{-10} \mathrm{~cm}^{-1} /\left(\operatorname{Torr}^{2} \mathrm{GHz}^{2} \mathrm{~K}^{\mathrm{n}}\right), \quad \mathrm{n}=-3.27(7)
$$


compared to

$$
C_{0}=1.877(13) 10^{-18} \mathrm{~cm}^{-1} /\left(\operatorname{Torr}^{2} \mathrm{GHz}^{2}\right), \quad \mathrm{n}=-3.27(7)
$$

for the fit with (4.14).

Not surprisingly, the value of the $C$ changes, as $C_{1}$ is scaled with respect to $C_{0}$ by $T^{n}$, but the uncertainty of the fit coefficient changes too, as small changes in the temperature power are translated into greater changes in $C$ in the (4.17) than in (4.14). Of course, when the correlation of the fit coefficients is taken into account appropriately, the uncertainty in the $\alpha_{\text {Nitr }}$, the only uncertainty with a direct physical meaning, stays exactly the same, no matter what scaling factor is used for the gas temperature.

While the good agreement of our nitrogen absorption data fit to a pressure squared model and the earlier good agreement of the absorption at each pressure fit to a frequency squared model is satisfying, it does not speak to the appropriate weighting of each of the points or the possible correlations of errors among them.

A direct, simultaneous global fit of the model to all of the data eliminates the purely statistical problems and provides a much more robust environment for the consideration of systematic effects relating either to the experiment or the assumed theoretical model. Nitrogen absorption measurements for different frequencies, pressures and temperatures have been fit to a single functional dependence of the form

$$
\alpha_{N i t r}(v, P, T)=C_{1} v^{C_{2}} P^{C_{3}} \theta^{C_{4}},
$$

where $\alpha_{\text {Nitrogen }}(v, P, T)$ is the nitrogen absorption at a frequency $v$, pressure $P$ and temperature $\quad T, \quad \theta=\frac{T}{300 K} ;$ and $C_{1}, C_{2}, C_{3}$, and $C_{4}$ are the fit coefficients. 
The total number of the cavity ringdown measurements for nitrogen was about $6 \times 10^{6}$, as the ringdown times for $\sim 6000$ frequencies were measured at $\sim 100$ pressures (including multiple scans at $\sim 50$ different pressures) for each of the 10 temperatures. Because of the slow change of the nitrogen absorption with frequency, averaging over some number of resonance frequencies can be used to speed up the fit and minimize the size of the data sets without degrading the fit integrity. 100 frequency points were averaged and included in the later fit, resulting in

$$
\alpha_{\text {Nitrogen }}(v, P, T)=2.89(6) v^{1.989(3)} P^{1.993(2)} \theta^{-3.257(3)} .
$$

Since both pressure and frequency dependences are quadratic within the experimental uncertainties, it is reasonable to fix both coefficients at 2.0, which results in the following expression for the nitrogen absorption:

$$
\alpha_{\text {Nitrogen }}(v, P, T)=1.90(1) v^{2} P^{2} \theta^{-3.257(3)}
$$

Results of the global fit are in agreement with the presented above results of the sequential fit, confirming the validity of the chosen parameterization and the error estimation. A subset of the nitrogen data (about $0.5 \%$ of the total data) is shown in Figure 4.14 along with the fitting function given by equation (4.22). 


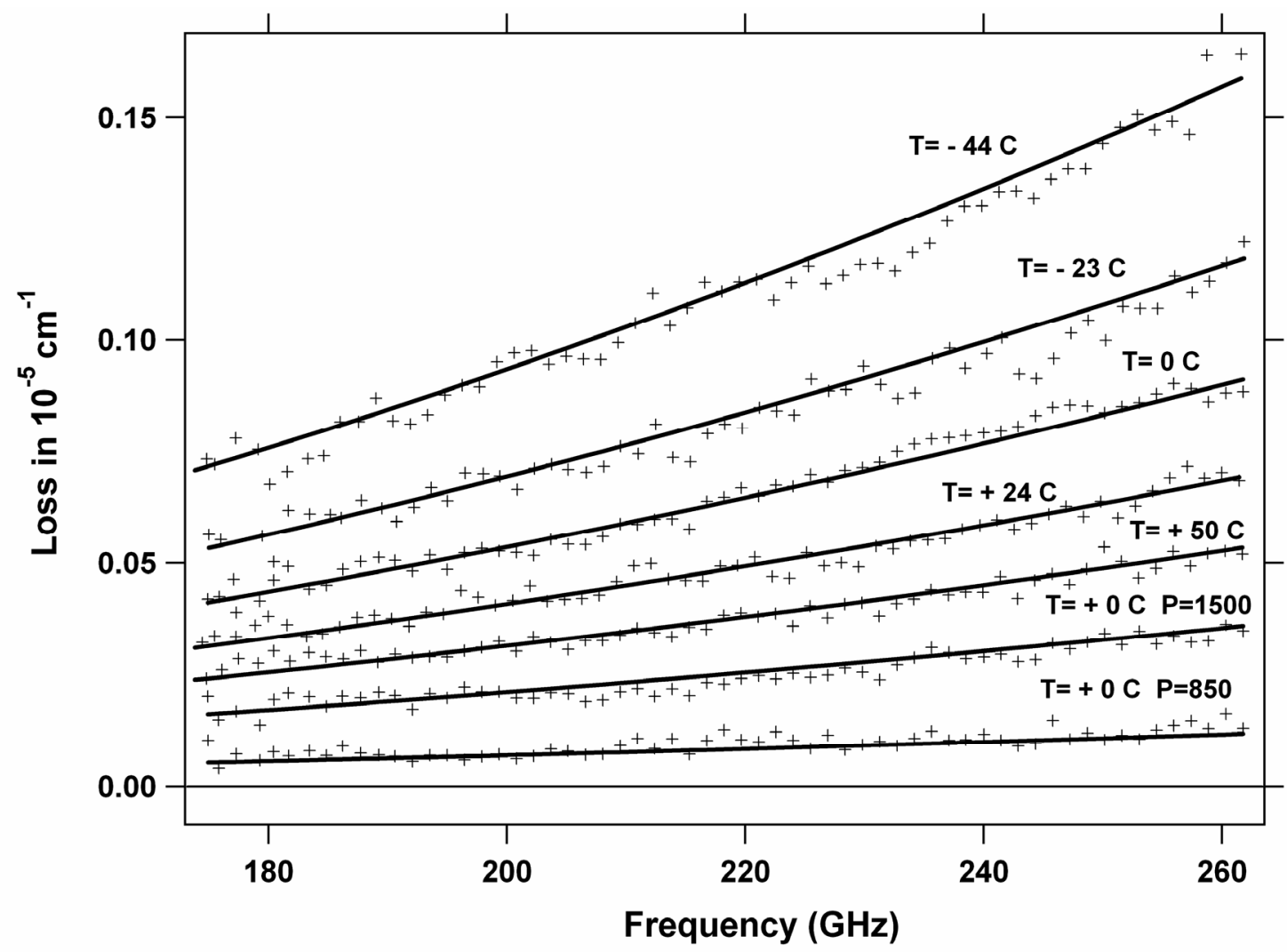

Figure 4.14: A subset of 100 point averaged nitrogen absorption data for a number of temperatures and pressures. Pressures are 2280 Torr (3 atm), unless otherwise noted. Solid line is a fit function given by equation (4.20).

Figure 4.14 shows only a small fraction of the nitrogen measurements. To further analyze the global fit performance, we can study the histogram of the residuals of the fit, shown in Figure 4.15. Smooth distribution of the residuals with no significant deviation from the normal distribution and no high deviation outliers is once again a sign that the nitrogen absorption is well described by the parameterization chosen above. 


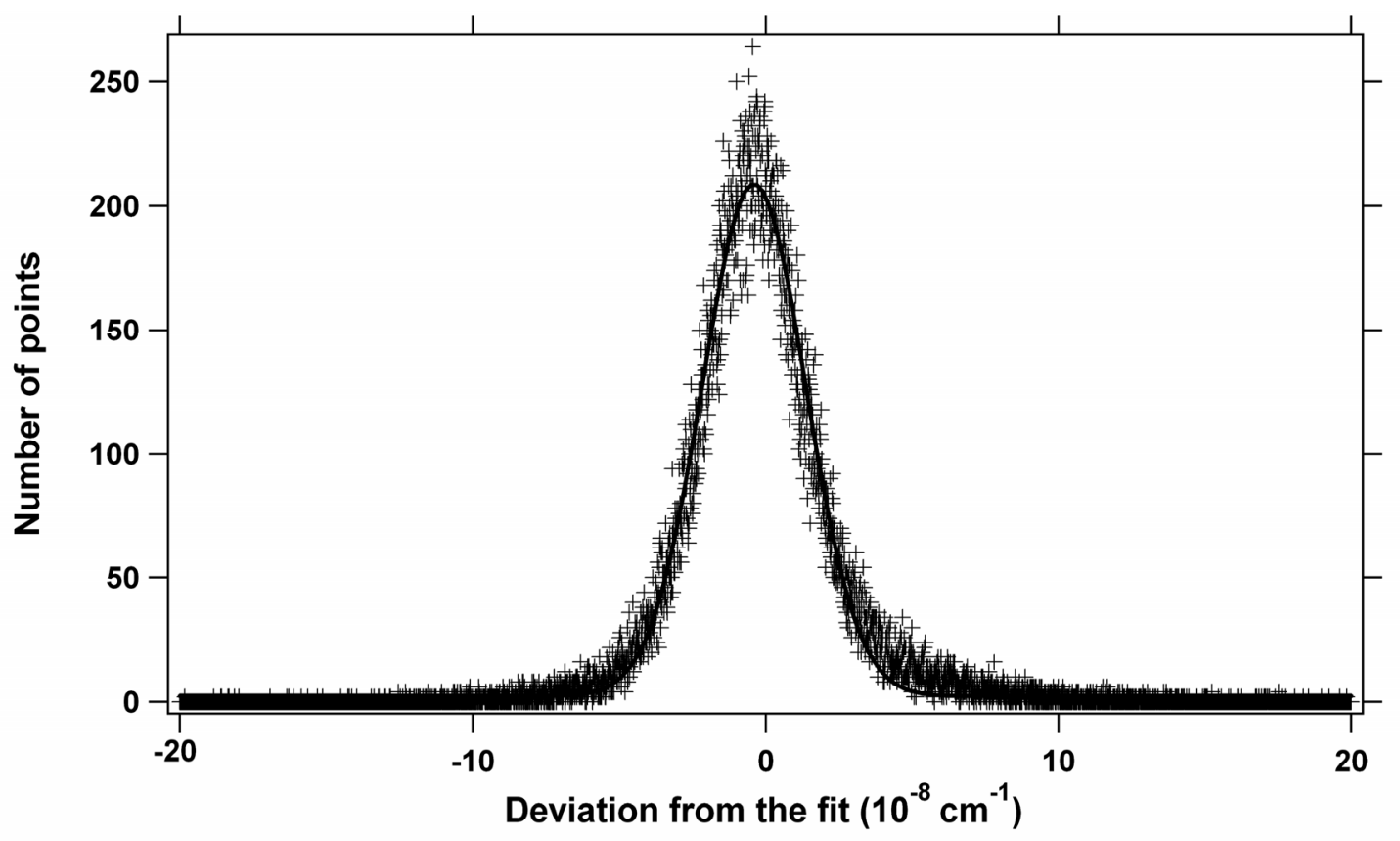

Figure 4.15: Histogram of the nitrogen global fit deviation.

Because of a simple scaling with pressure, the nitrogen collision induced absorption can be measured at pressures much higher than the atmospheric and later scaled down. Since the 1962 Birnbaum and Maryott ${ }^{68}$ measurements at $24.35 \mathrm{GHz}$, $295 \mathrm{~K}$ and pressures up to $103 \mathrm{~atm}$, the nitrogen microwave absorption at high pressures has been studied by a number of groups. In 1968 , Ho et al. ${ }^{69}$ published their measurements of nitrogen absorption at $9.26 \mathrm{GHz}, 238-493 \mathrm{~K}$ temperature range and pressures up to $135 \mathrm{~atm}$. Another study of the nitrogen absorption as a function of temperature was published by Dagg et al. ${ }^{70}$ in 1975 for $70 \mathrm{GHz}$, temperature range of 208-333 $\mathrm{K}$ and pressures as high as $300 \mathrm{~atm}$. In addition to these two temperature dependence studies, high pressure measurements at room temperature were made by Dagg et al. ${ }^{71}$ at $140 \mathrm{GHz}$ and Occelli et al. ${ }^{72}$ at eight frequencies below $1 \mathrm{THz}$, including $152 \mathrm{GHz}, 316 \mathrm{GHz}$, and $403 \mathrm{GHz}$. 
Figure 4.16 shows how our measurements compare with the temperature dependent measurements of other groups, given by

Ho et al. ${ }^{69}$

$$
C(T)=\frac{\alpha_{\text {Nitr }}}{P^{2} v^{2}}=1.34(8) \theta^{-4.5(2)}
$$

Dagg et al. ${ }^{70}$

$$
C(T)=\frac{\alpha_{\text {Nitr }}}{P^{2} v^{2}}=1.868(26) \theta^{-3.55(12)}
$$

our measurements

$$
C(T)=\frac{\alpha_{\text {Nitr }}}{P^{2} v^{2}}=1.877(13) \theta^{-3.27(7)}
$$

Our room temperature measurements are in excellent agreement with results of Dagg et al. ${ }^{70}$ At low temperatures our data is systematically smaller, which leads to different temperature exponents in 4.24 and 4.25. Although the uncertainties in the temperature exponents do not overlap, the individual measurement uncertainties do overlap in the common temperature range.

The absolute value of the absorption measured by Ho et al. ${ }^{69}$ at room temperature is about $30 \%$ smaller than ours. Their temperature exponent of $-4.5(2)$ is much steeper than ours. This is possibly due to the large errors of their higher (up to $493 \mathrm{~K})$ temperature measurements, where absorption is at least five times smaller than at room temperature. It is especially important since their measurement were made at $9.26 \mathrm{GHz}$, with absorption almost 60 times smaller than for the $70 \mathrm{GHz}$ measurements of Dagg et al. 


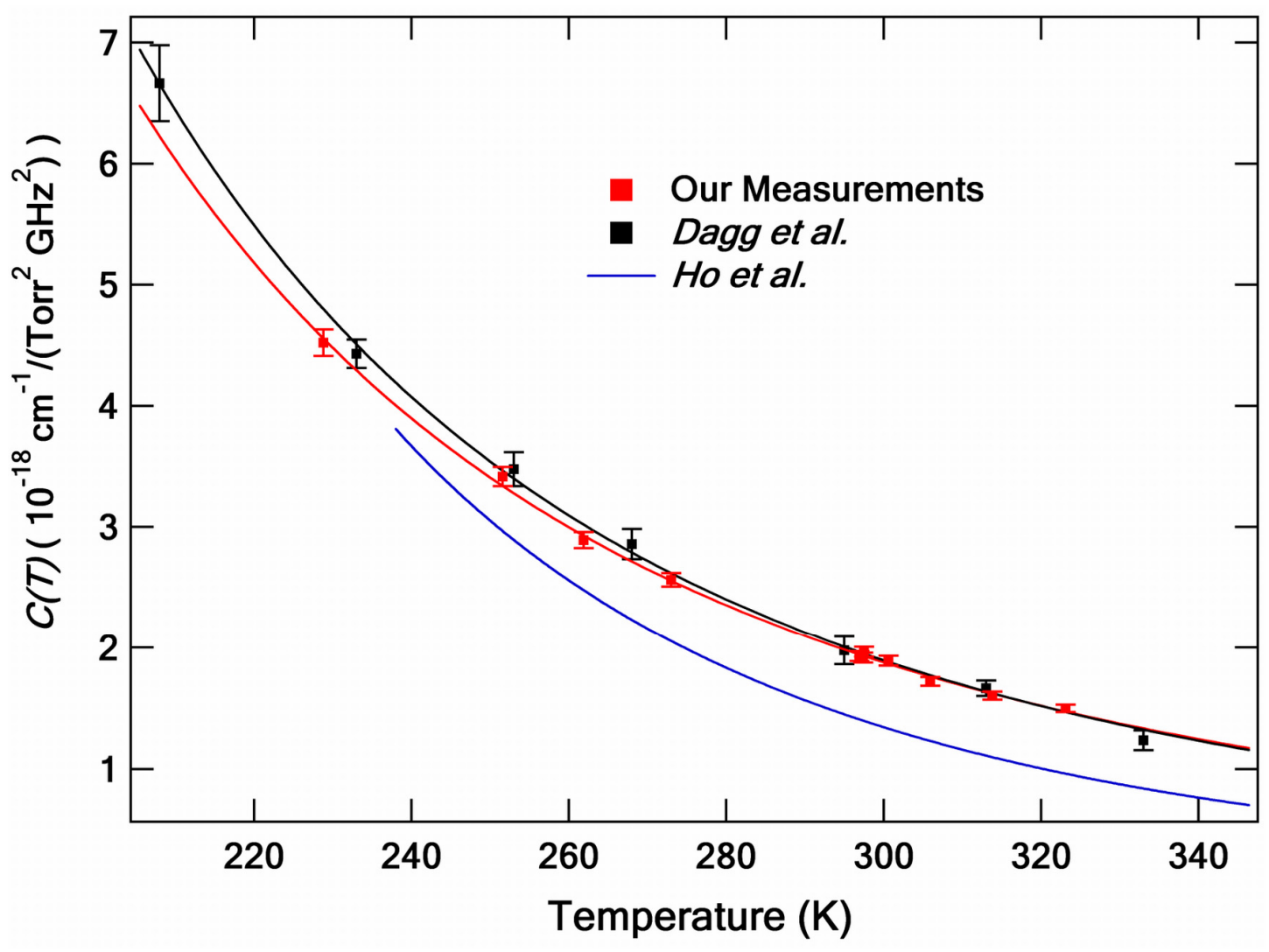

Figure 4.16: Comparison of our measurements of the $C(T)=\frac{\alpha_{\text {Nitr }}}{P^{2} v^{2}}$ with measurements made by Ho et.al ${ }^{69}$ and Dagg et al. ${ }^{70}$

A comparison of room temperature nitrogen absorption measurements by different groups is shown in Figure 4.17. With exception of the early measurements by Birnbaum and Maryott ${ }^{68}$ in 1962 (no uncertainties are presented or discussed) and Ho et al. $^{69}$ in 1968 (heavy reliance on the high temperature data), our room temperature results are consistent with the high pressure measurements by other groups. This is a good test of both the system performance and the validity of the nitrogen frequency squared absorption dependence, since these measurements were performed at frequencies from 70 to $403 \mathrm{GHz}$, with the absorption to frequency squared ratio staying constant within the error bars. 


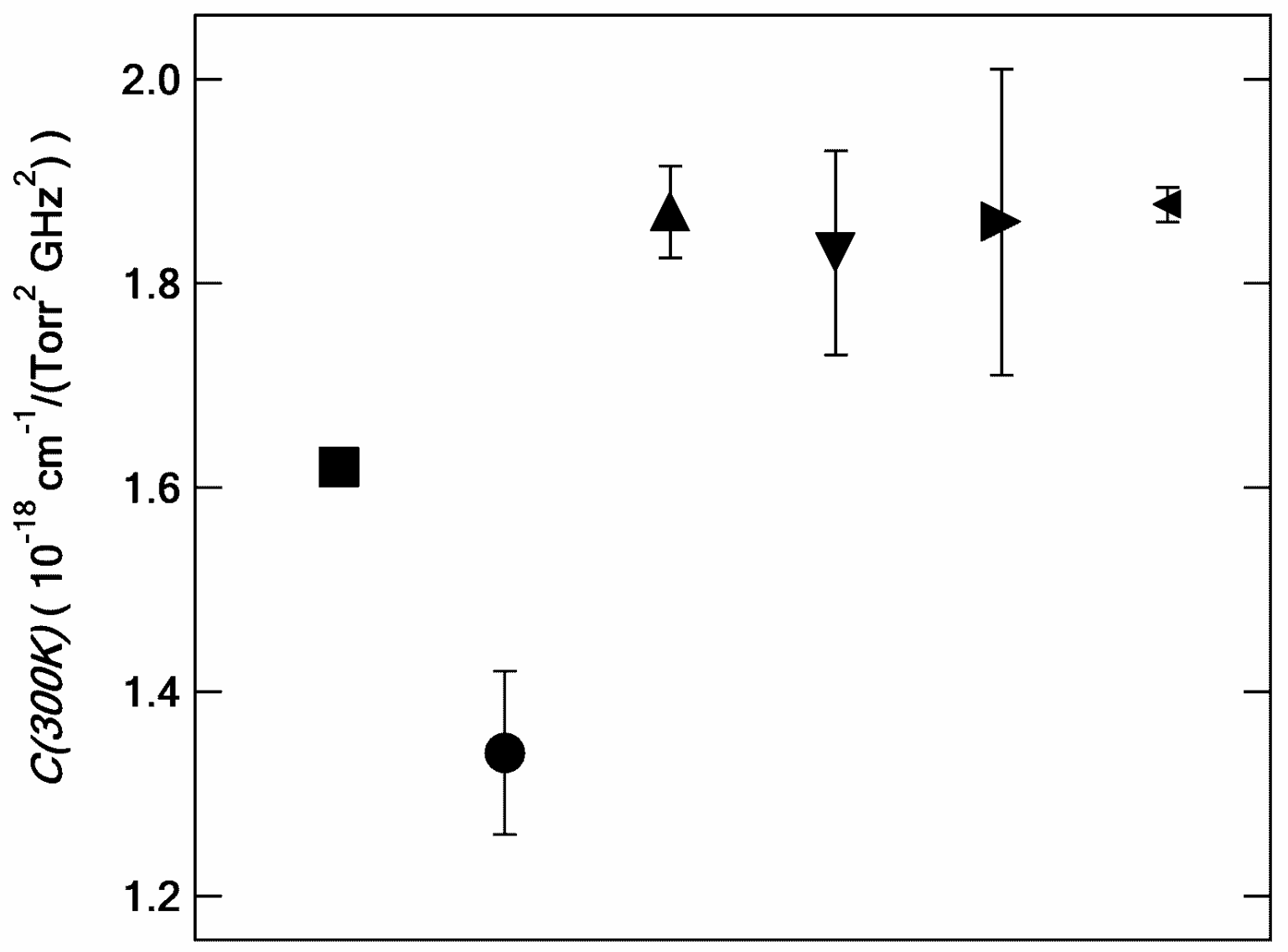

Figure 4.17: Room temperature nitrogen absorption measurements $C(300 K)=\frac{\alpha_{\text {Nitr }}(300 K)}{P^{2} v^{2}}$ by Birnbaum and Maryott ${ }^{68}$ at $24.35 \mathrm{GHz}(\boldsymbol{\square})$,

Ho et al. ${ }^{69}$ at $9.26 \mathrm{GHz}(\bullet)$, Dagg et al. ${ }^{70}$ at $70 \mathrm{GHz}(\boldsymbol{\Delta})$, Dagg et al. ${ }^{71}$ at $140 \mathrm{GHz}(\boldsymbol{\nabla})$, Occelli et al. ${ }^{72}$ at $152 \mathrm{GHz}, 316 \mathrm{GHz}$, and $403 \mathrm{GHz}$, averaged ( $(\mathbf{)}$ ) comparison with our measurements (४). All measurements are scaled to $300 \mathrm{~K}$ using a 3.4 temperature exponent. 


\subsubsection{Oxygen absorption}

Oxygen, as described in section 2.4.2, has microwave absorption lines due to its permanent magnetic dipole moment. There are no oxygen lines of the most abundant isotope $\left(\mathrm{O}_{16} \mathrm{O}_{16}\right)$ in the $170-260 \mathrm{GHz}$ range, and the oxygen absorption in this frequency range comes from the far wings of the outside lines, the constant in frequency Debye absorption and the oxygen collision induced absorption. There is also a small contribution from the $233.95 \mathrm{GHz} \mathrm{O}_{18} \mathrm{O}_{16}(\sim 0.4 \%$ abundance $)$ absorption line.

There was no noticeable difference in the measured absorption between the Extra Dry Oxygen (99.6\% pure) and the much more expensive Ultra High Purity Oxygen (99.993\% pure), both ordered from Praxair, so the former was used in all our oxygen measurements.

Because our frequency range is far away from the $\mathrm{O}_{16} \mathrm{O}_{16}$ line centers, absorption contribution from these lines strongly depends on the line parameters like line positions, pressure broadening and line mixing coefficients. Oxygen absorption close to the line centers have been extensively studied by the scientific community both for the pure oxygen and the dry air. Liebe has used the modified by Rozenkranz $^{73}$ Van Vleck-Weiscopf line shape function to fit his dry air measurements around $60 \mathrm{GHz}$ and to derive the line parameters to be used in his Millimeter-Wave Propagation Model (MPM), with additions and improvements, published as the MPM89 $^{28}$, MPM92 $2^{38}$ and MPM93 ${ }^{37}$. Although the line pressure broadening and the line mixing parameters of the above models are given for dry air, it is useful to see how well these models predict the pure oxygen absorption far from the line centers. 
To do this, the Liebe model absorption was scaled accordingly to the $21 \%$ oxygen fraction in the dry air, while all the line parameters were kept the same. Figure 4.18 shows our experimentally measured room temperature $3 \mathrm{~atm}$ of oxygen absorption along with results of the MPM 89, 92 and 93 for 2280 Torr and $24{ }^{\circ} \mathrm{C}$. The Debye absorption stayed the same for all MPM models, while the line by line contribution was changed in each successive model to better represent the available data.

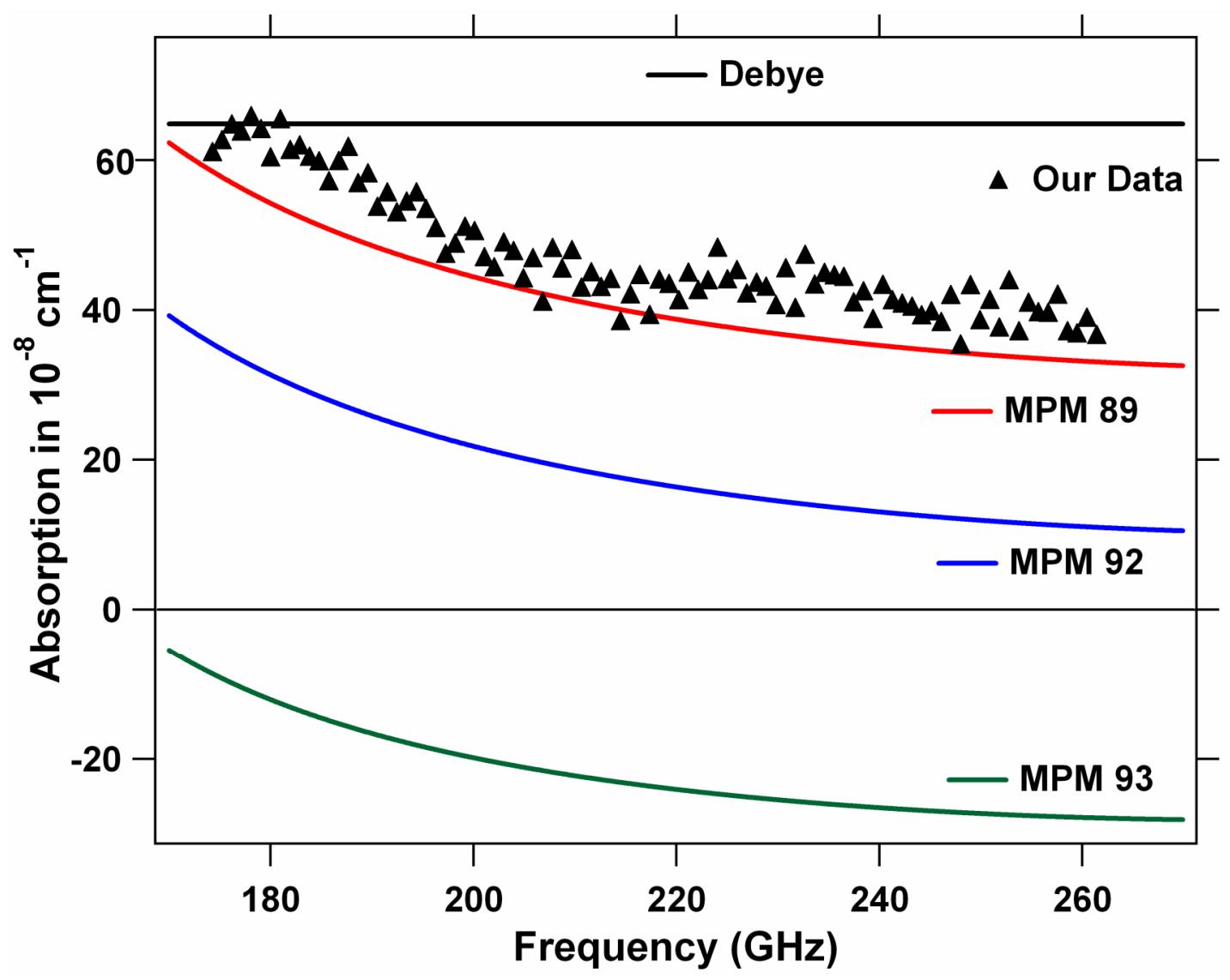

Figure 4.18: Comparison of our room temperature 3 atm oxygen absorption measurements with the Liebe MPM89, MPM92 and MPM93 models

Although the MPM89 prediction is in a reasonable agreement with our data, it is unclear if it is more than a coincidence since with each new model the predicted absorption gets further from the measured. For each model the total line by line contribution is always negative, as can be seen from the fact that the Debye 
absorption alone is greater than the total absorption. This comes from the fact that in the fitting algorithm used in the Liebe models the line parameters varied freely to give the best fit to the absorption data close to the absorption lines, without an explicit constrain on the absorption behavior as the frequency approaches infinity. ${ }^{74}$ Although this "negative absorption" is very small compared to the absorption around the line centers, it is clearly nonphysical. The difference in the pure oxygen line parameters and the dry air parameters can not be the only reason for the above disagreement, as using the experimentally measured pure oxygen line parameters ${ }^{74-76}$ did not improve the model agreement with the experiment.

Because of the above reasons, instead of attempting to describe our experimental results in terms of the measured line parameters, we decided to use an empirical function to fit our data. This function was to include the constant Debye absorption, oxygen lines far wings contribution and the oxygen collision induced absorption in the form:

$$
\alpha_{\text {Oxygen }}(v, P, T)=\left(C_{\text {Debye }}(T)+\frac{C_{\text {Lines }}(T)}{(v-120)^{2}}+C_{C I A}(T) v^{2}\right) P^{2}+\alpha_{0_{18} O_{16}}(v, P, T) .
$$

The center frequency in the line contribution term was arbitrary chosen as $120 \mathrm{GHz}$. Changing this frequency by $\pm 10 \mathrm{GHz}$ changes the values of the other fit coefficients but does not significantly change the quality of the fit. We have also neglected the pressure dependence of $C_{\text {Lines }}$, as even at $180 \mathrm{GHz}$ and $3 \mathrm{~atm}$ pressure, the correction $\left(\frac{\Delta v}{v-120}\right)^{2}$ is less than $0.5 \%$ for pure oxygen pressure broadened lines. Removing the CIA term from the fitting function did not seem to degrade the fit and this term was omitted in the later analysis in order to keep the number of coefficients at 
minimum. This means that the oxygen CIA term is much smaller than that of the nitrogen, which is consistent with the theoretical analysis of Boissoles et al. ${ }^{27}$ and the far infrared measurements by Bosomworth et al. ${ }^{77}$.

Room temperature oxygen absorption measurements for a number of different pressures are shown in Figure 4.19 along with the fitting function from equation (4.27).

$$
\alpha_{\text {Oxygen }}(v, P, T)=\left(C_{1}(T)+\frac{C_{2}(T)}{(v-120)^{2}}\right) P^{2}+\alpha_{0_{18} O_{16}}(v, P, T)
$$

The pressure broadening and the line intensity parameters for the $\mathrm{O}_{18} \mathrm{O}_{16}$ line were themselves parameters of the fit according to

$$
\alpha_{o_{18} o_{16}}(v, P, T)=C_{3} \frac{P^{2}}{(233.946-v)^{2}+\left(C_{4} P\right)^{2}} .
$$

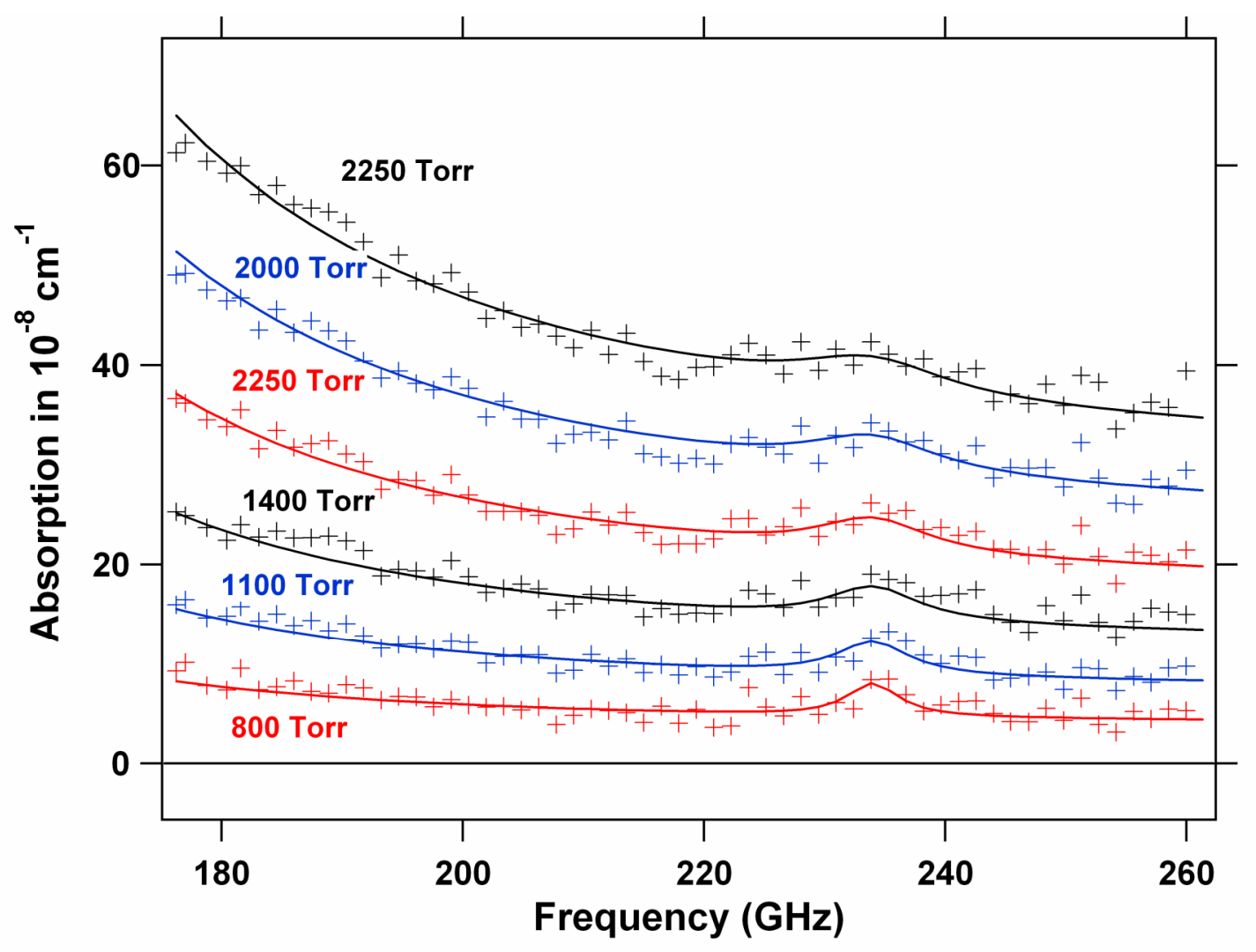

Figure 4.19: Room temperature oxygen absorption measurements. Solid lines are results of the fit with parameterization given by (4.27). 
Although each term in the chosen parameterization has a physical meaning, it is worth reiterating that we use equation (4.27) only to give an empirical way to calculate the absorption of oxygen for a given pressure and temperature. We deliberately removed the subscripts in the fitting coefficients, as both $\mathrm{C}_{1}$ and $\mathrm{C}_{2}$ depend, for example, on the choice of the center frequency in the fitting function (120 GHz in 4.27).

Results of the fits for different temperatures are given in Table 4.3.

\begin{tabular}{ccccc} 
Temperature $(\mathrm{K})$ & $\mathrm{C}_{1}(\mathrm{~T})$ & $\mathrm{C}_{2}(\mathrm{~T})$ & $\mathrm{C}_{3}(\mathrm{~T})$ & $\mathrm{C}_{4}(\mathrm{~T})$ \\
& $\begin{array}{c}10^{-14} \\
\mathrm{~cm}^{-1} / \mathrm{Torr}^{2}\end{array}$ & $\begin{array}{c}10^{-10} \\
\mathrm{~cm}^{-1} / \mathrm{Torr}^{2}\end{array}$ & $\begin{array}{c}10^{-12} \\
\mathrm{~cm}^{-1}\end{array}$ & $\begin{array}{r}10^{-3} \\
1 / \mathrm{Torr}\end{array}$ \\
252.0 & 9.61 & 3.87 & 1.62 & 5.35 \\
272.8 & 6.94 & 2.73 & 1.79 & 5.28 \\
296.7 & 5.69 & 2.26 & 0.325 & 3.13 \\
296.8 & 6.38 & 1.98 & 0.520 & 4.34 \\
302.9 & 5.32 & 2.19 & 0.086 & 1.58 \\
323.6 & 4.44 & 1.65 & 0.156 & 1.85 \\
\hline
\end{tabular}

Table 4.3: Oxygen absorption fitting coefficients.

Figure 4.20 shows how $C_{1}(T)$ varies with temperature, along with the fit to the power law temperature dependence in the form

$$
C_{1}(T)=C_{1}\left(\frac{T}{300 K}\right)^{-n}
$$

The fit results in $C_{1}=5.6(2) 10^{-14} \mathrm{~cm}^{-1} /$ Torr $^{2}, n=3.0(3)$. It is interesting to note that 
the temperature exponent is in agreement with $n=3.0$ used in the MPM89 and $n=2.8$ used in MPM92 and MPM93. The value of $C_{1}$ is about one half of the dry air Debye absorption from the MPM models.

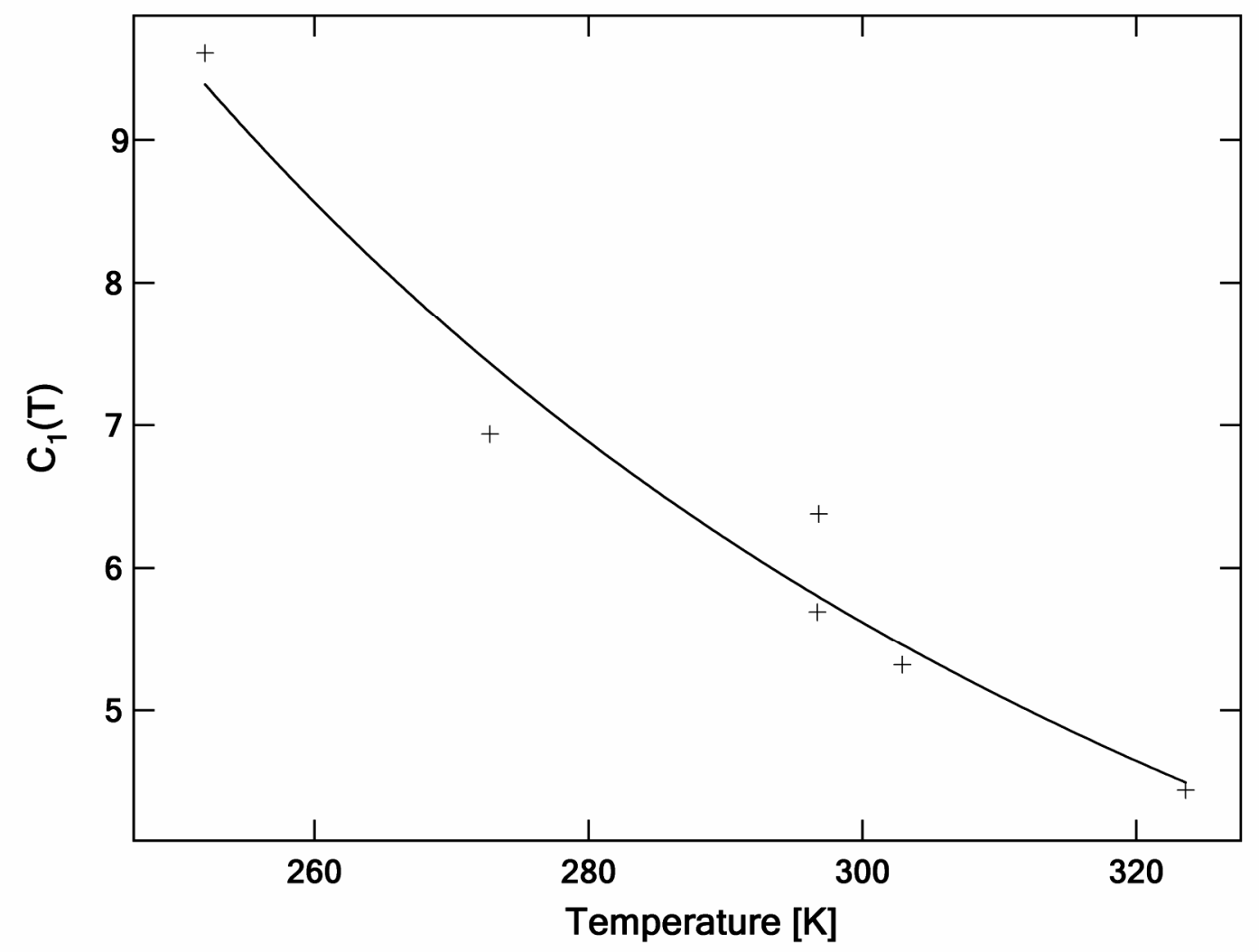

Figure 4.20: The fit coefficient $C_{1}(T)$ for the constant in frequency oxygen absorption term temperature dependence.

Comparing the two room temperature measurements taken on different days gives insight on the fit performance in separating the line absorption contribution from the constant in frequency Debye-like term. Although the actual predicted absorption is within $3 \%$ for both days, the coefficients $C_{1}(T)$ and $C_{2}(T)$ vary by as much as $10 \%$.

The accuracy of the fitting coefficients as well as the general understanding of the oxygen absorption parameterization should improve if the temperature range is 
broadened and the frequency coverage is extended to include at least the nearest oxygen absorption lines. Our current oxygen absorption measurements were performed as a preliminary study to aid in the dry air absorption analysis. There is also an indication ${ }^{78}$ that at higher temperatures the oxygen absorption starts to increase. Although in this work the pure oxygen absorption was not studied as extensively as the nitrogen and the dry air absorptions because of its small contribution to the atmospheric absorption at this frequency range, the instrument is well suited for further studies.

\subsubsection{Dry air absorption}

The composition of standard dry air is defined by molar percentage as $78.09 \%$ nitrogen, $20.95 \%$ oxygen, $0.93 \%$ argon, and $0.03 \%$ of carbon dioxide. ${ }^{23}$ In this work we have studied the dry air composed of nitrogen (79\%) and oxygen (21\%) only, neglecting contributions from carbon dioxide (no rotational spectrum, small percentage) and argon (atomic gas, no absorption in the microwave). The same quality gases were used for the dry air measurements as for the pure nitrogen and oxygen measurements.

Pure oxygen, nitrogen and dry air absorptions at $3 \mathrm{~atm}$ at room temperature are shown in Figure 4.21. It is clear that the dry air absorption variation with frequency can not be described by a simple quadratic dependence as was the case for nitrogen. 


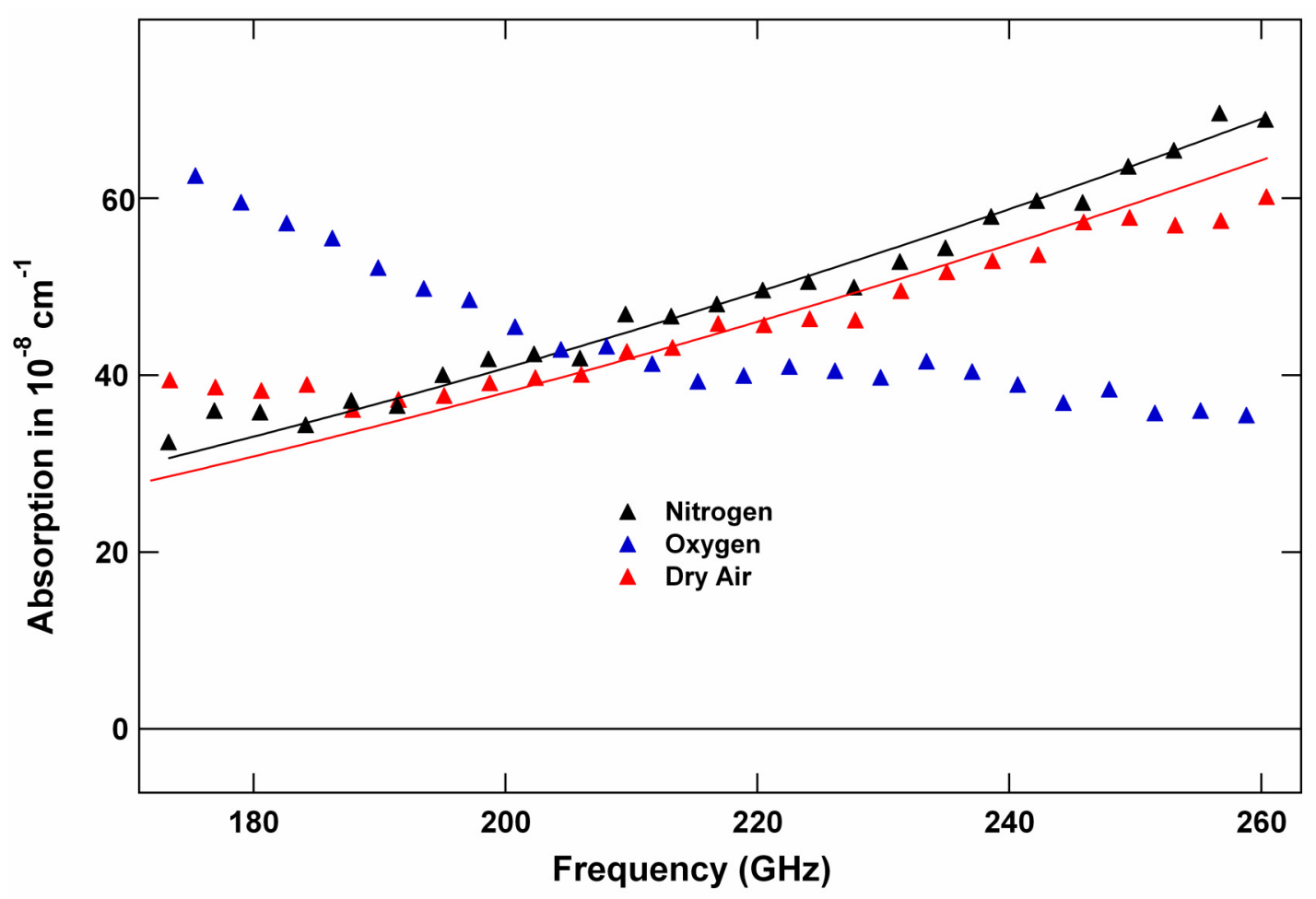

Figure 4.21: 250 point average of the room temperature absorption for 3 atm of nitrogen, oxygen, and dry air. Straight lines are fits to a simple quadratic in frequency dependence for nitrogen and the dry air.

As a starting point, a combination of two terms, constant and quadratic in frequency, was used to parameterize the dry air absorption as

$$
\alpha_{D r y}(v, P, T)=\left(C_{1}(T)+C_{2}(T) v^{2}\right) P^{2} .
$$

The constant in frequency term is the oxygen Debye absorption, the quadratic term is the sum of the nitrogen-nitrogen, nitrogen-oxygen, oxygen-nitrogen, and the oxygenoxygen CIA contributions. The oxygen lines absorption was not included in 4.30 and instead only absorption for frequencies of $180 \mathrm{GHz}$ and above, for which the oxygen lines contribution is negligible, was used in the fit. Because of the limited frequency coverage, the coefficients $C_{1}(T)$ and $C_{2}(T)$ are correlated and could not be separated with high accuracy. Results for $C_{1}(T)$ as a function of temperature are 
shown in Figure 4.22 along with the Debye absorption of the Liebe MPM93, given by

$$
\alpha_{\text {Debye }}^{M P M}(v, P, T)=2.5410^{-14} P^{2}\left(\frac{T}{300}\right)^{-2.8} \mathrm{~cm}^{-1} / \text { Torr }^{2} \text {. }
$$

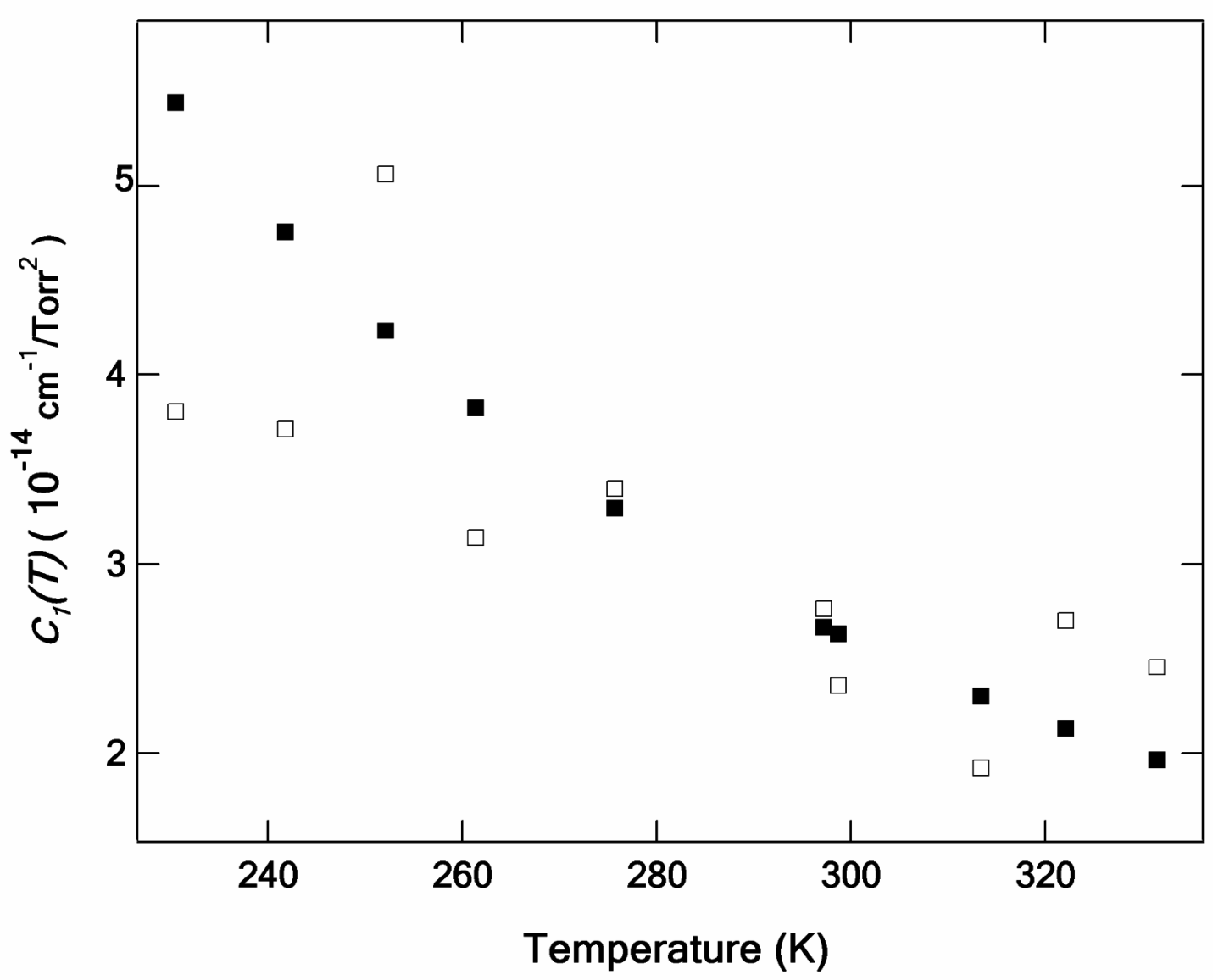

Figure 4.22: Comparison of the Debye absorption of the Liebe MPM93 model ( $\square$ ) with results of the fit of our data with equation (4.30) (口)

Because no systematic deviation was found, the Liebe's parameterization was later used to subtract the Debye absorption from our measurements to study the behavior of the dry air collision induced absorption as

$$
\alpha_{\text {Dry }}^{C I A}(v, P, T)=\alpha_{D r y}(v, P, T)-\alpha_{\text {Debye }}^{M P M}(v, P, T)=C(T) v^{2} P^{2}
$$


Uncertainties in the total dry air absorption measurements can be calculated in the same manner as it was done for nitrogen, assuming $\pm 1{ }^{0} \mathrm{C}$ uncertainty in the temperature and the relative pressure uncertainty of $0.5 \%$.

$$
\frac{\Delta \alpha_{D r y}(v, P, T)}{\alpha_{D r y}(v, P, T)}=0.01+\frac{3.3}{T}
$$

Since we use the exact value for the Debye absorption subtraction, the absolute uncertainty in the $\alpha_{D r y}^{C I A}(\nu, P, T)$ is the same as the absolute uncertainty in the total dry air absorption

$$
\Delta \alpha_{D r y}^{C I A}(v, P, T)=\Delta \alpha_{D r y}(v, P, T)=\left(0.01+\frac{3.3}{T}\right) \alpha_{D r y}(v, P, T)
$$

From this, the uncertainty in $C(T)$ from 4.32 can be estimated as

$$
\Delta C(T)=\left(0.01+\frac{3.3}{T}\right)\left(C(T)+\frac{\alpha_{\text {Debye }}^{M P M}(T, P=3 \mathrm{~atm})}{220^{2}}\right),
$$

where the Debye absorption is scaled by the average scan frequency squared to account for the difference in the frequency dependence.

Results of the fits are presented in Table 4.4 and Figure 4.23. Figure 4.23 also shows the fit of the collision induced absorption temperature behavior to a power law, resulting in

$$
C(T)=1.30(4) 10^{-18}\left(\frac{T}{300 K}\right)^{-3.35(11)} \mathrm{cm}^{-1} /\left(\operatorname{Torr}^{2} \mathrm{GHz}^{2}\right)
$$


We can now combine equations (4.31), (4.32), and (4.36) to obtain the final expression for the dry air absorption

$\alpha_{D r y}(v, P, T)=2.5410^{-14} P^{2}\left(\frac{T}{300}\right)^{-2.8}+1.30(4) 10^{-18}\left(\frac{T}{300}\right)^{-3.35(11)} v^{2} P^{2} \mathrm{~cm}^{-1},(4.37)$ where $P$ is the total dry air pressure in Torr, $v$ is the frequency in $\mathrm{GHz}$ and $T$ is the temperature in $\mathrm{K}$.

\begin{tabular}{cc} 
Temperature $(\mathrm{K})$ & $C(T)\left(10^{-18} \mathrm{~cm}^{-1} /\left(\operatorname{Torr}^{2} \mathrm{GHz}^{2}\right)\right)$ \\
230.5 & $3.02(10)$ \\
241.8 & $2.74(9)$ \\
252.1 & $2.47(8)$ \\
261.4 & $1.99(6)$ \\
275.7 & $1.69(5)$ \\
297.2 & $1.38(4)$ \\
298.7 & $1.32(4)$ \\
313.4 & $1.10(3)$ \\
\hline
\end{tabular}

Table 4.4: Dry air collision induced absorption coefficient $C(T)$. 


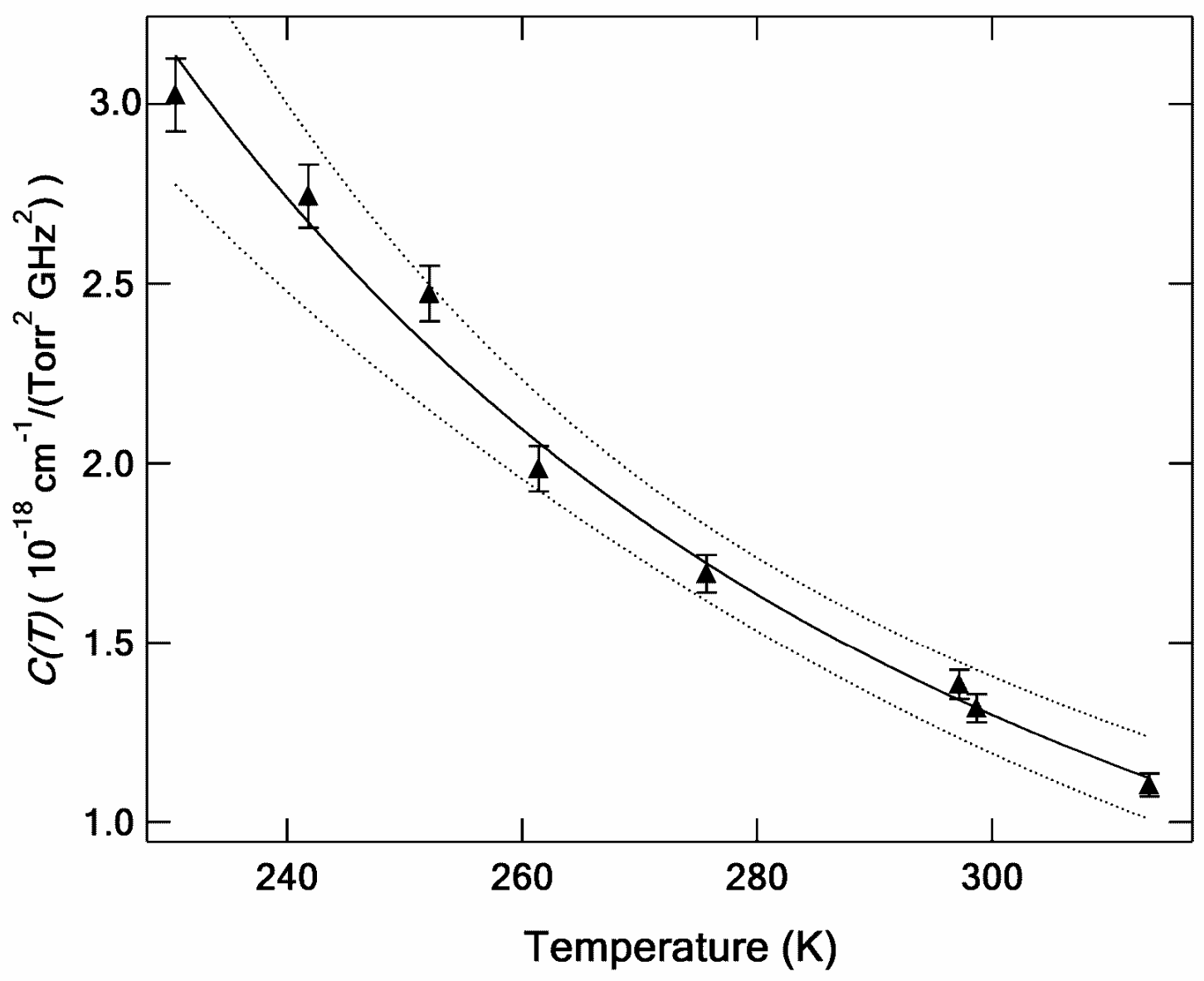

Figure 4.23: Temperature dependence of the dry air collision induced absorption. Solid line is a result of the temperature power law fit. Dotted lines are the $3 \sigma$ confidence bands.

A subset of the dry air data for a number of temperatures and pressures is shown in Figure 4.24 along with the fitting function given by equation (4.37). 


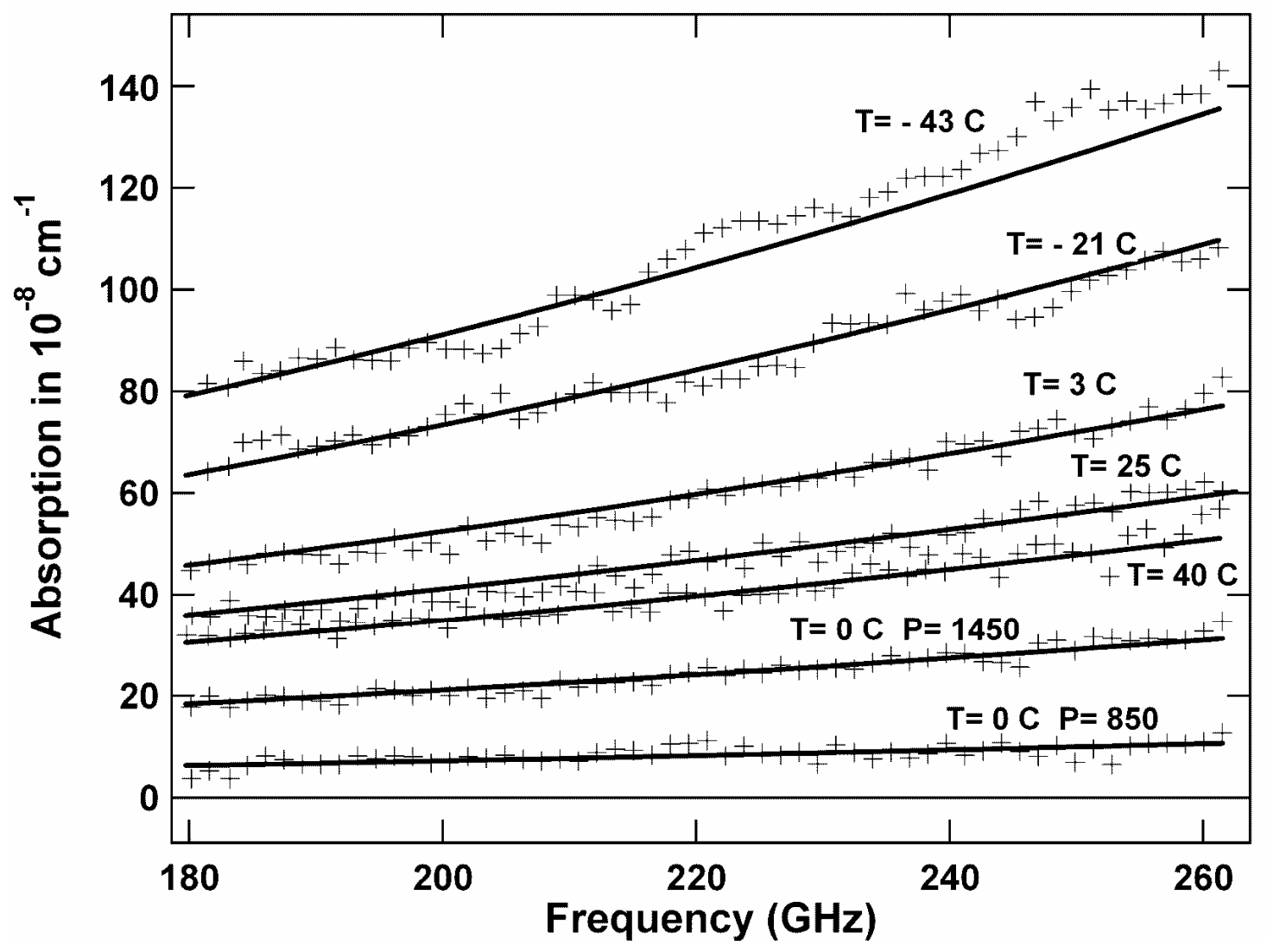

Figure 4.24: A subset of 100 point averaged dry air absorption data for a number of temperatures and pressures. Pressures are 2280 Torr $(3 \mathrm{~atm})$, unless otherwise noted. Solid line is a fit function given by equation (4.20).

Comparison of the absorption data with the results of the fit shows that our measurements are well described by the MPM parameterization of the dry air absorption. The actual values of parameters are quite different though. Liebe collision induced absorption is given by

$$
\alpha_{C I A}^{M P M}(v, P, T)=1.006 v^{2} P^{2}\left(\frac{T}{300}\right)^{-3.5} 10^{-18} \mathrm{~cm}^{-1} .
$$

At $300 \mathrm{~K}$,

$$
\alpha_{C I A}^{\text {Measured }} \approx 1.30 \alpha_{C I A}^{M P M}
$$

This is in agreement with the 1.29 value used by Pardo et al. ${ }^{79}$ to fit their atmospheric transmission spectra, obtained on Mauna Kea, Hawaii in 1998 and 1999. Figure 4.25 
shows our room temperature dry air absorption measured at $3 \mathrm{~atm}$, along with our fit and the MPM89 prediction, with and without the 1.3 scaling coefficient. Because of the reasons discussed in the oxygen absorption section above, estimation of the oxygen lines contribution to the dry air absorption at our frequency is problematic. Excluding these line contributions from the MPM 89 and multiplying the CIA absorption by 1.3 provides a very good agreement with our measurements data.

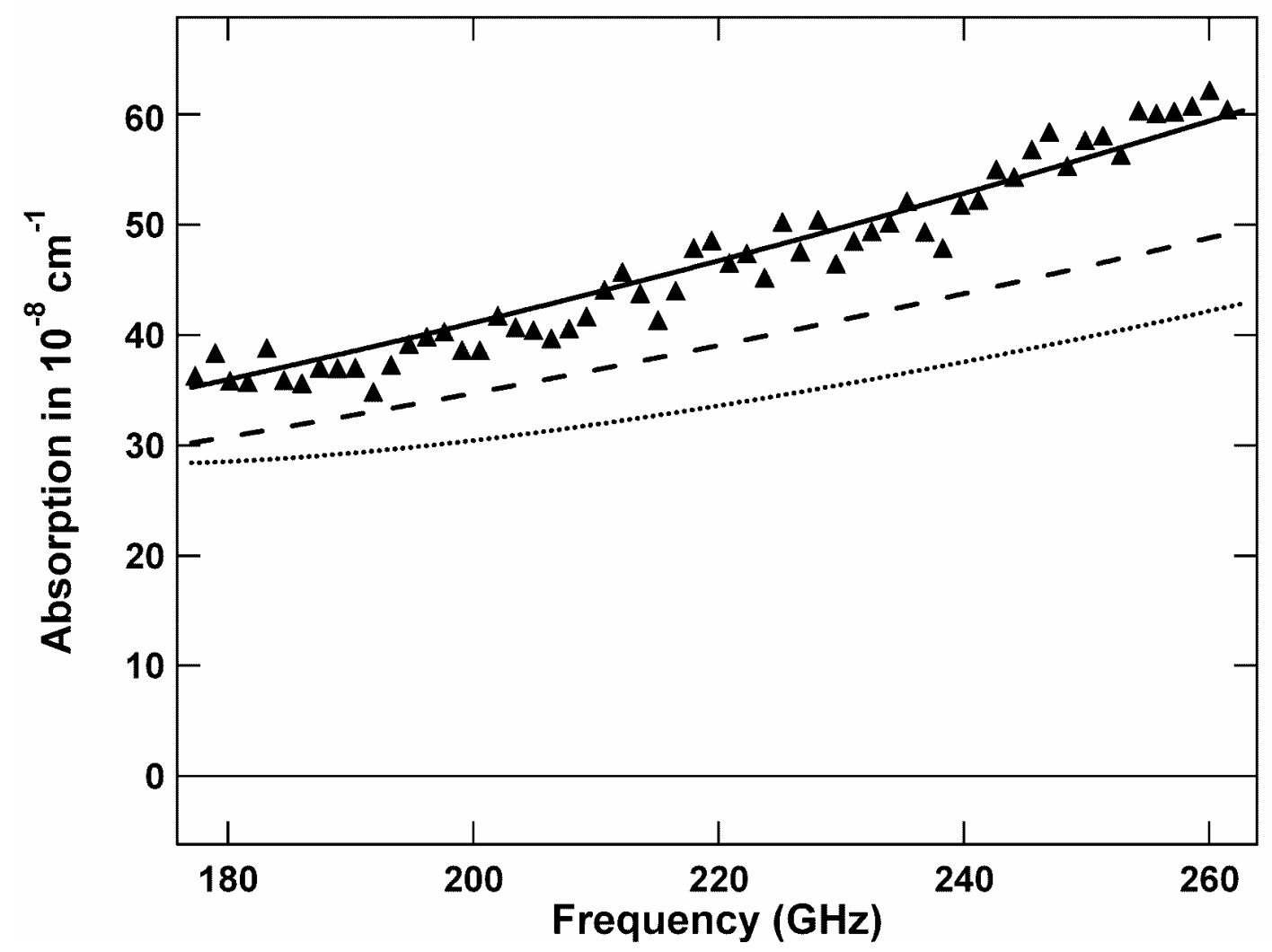

Figure 4.25: 100 point averaged room temperature absorption of 3 atm of dry air $(\boldsymbol{\Delta})$. Liebe MPM89 prediction including the oxygen lines contributions ( $\cdots)$, without oxygen lines (---), and without oxygen lines, but with the CIA contribution multiplied by 1.3 (solid line). The solid line is also the result of the fit of our data, given by 4.37 . 
Our experimental temperature exponent values for the nitrogen and the dry air absorptions are $-3.26(4)$ and $-3.35(11)$, respectively. The fact that the temperature dependences are close is consistent with the theoretical analysis by Boissoles et al. ${ }^{27}$ Liebe uses the -3.5 exponent from Stone et ll. $^{25}$ Other literature values include $-3.55(12)$ by Dagg et al. ${ }^{70}$ and $-4.5(2)$ by Ho et al. ${ }^{69}$, both measured for nitrogen CIA at high pressures.

We can now compare the nitrogen and the dry air collision induced absorption measurements. In general, the CIA for the dry air can be written as the sum of three terms due to collisions of nitrogen with nitrogen, oxygen with oxygen, and nitrogen with oxygen.

$$
\begin{gathered}
\alpha_{C I A}^{\text {DryAir }}=\alpha_{C I A}\left(N_{2}-N_{2}\right) 0.79^{2}+\alpha_{C I A}\left(O_{2}-O_{2}\right) 0.21^{2} \\
+\alpha_{C I A}\left(N_{2}-O_{2}\right) 0.790 .21
\end{gathered}
$$

From the far infrared measurements of the oxygen and nitrogen CIA it is known that $\alpha_{C I A}\left(O_{2}-O_{2}\right)$ is almost a factor of 10 smaller than $\alpha_{C I A}\left(N_{2}-N_{2}\right)^{80}$. Multiplied by $\left(\frac{0.21}{0.79}\right)^{2}$ to take into account the relative abundance, the contribution from the oxygen-oxygen collisions to the dry air CIA becomes less than $1 \%$ of the nitrogennitrogen CIA. If we neglect the pure oxygen CIA, we can use equations (4.19), (4.36), and (4.40) to calculate that

$$
\frac{\alpha_{C I A}\left(N_{2}-O_{2}\right)}{\alpha_{C I A}\left(N_{2}-N_{2}\right)}=\frac{\alpha_{C I A}^{\text {DryAir }}-\alpha_{C I A}\left(N_{2}-N_{2}\right) 0.79^{2}}{0.790 .21 \alpha_{C I A}\left(N_{2}-N_{2}\right)} \approx 0.44(13)
$$

The uncertainty in (4.41) was calculated using the uncertainties in (4.19) and (4.36). Possible systematical error in the estimation of the Debye absorption contribution could be one of the reasons for the disagreement of our result for 
$\frac{\alpha_{C I A}\left(N_{2}-O_{2}\right)}{\alpha_{C I A}\left(N_{2}-N_{2}\right)}$ with the 1.249 , calculated by Boissoles et al. ${ }^{27}$ If the Debye absorption is decreased by $20 \%$, the $\frac{\alpha_{C I A}\left(N_{2}-O_{2}\right)}{\alpha_{C I A}\left(N_{2}-N_{2}\right)}$ is doubled, which demonstrates how sensitive this ratio is to the changes in the chosen Debye absorption contribution.

Furthermore, we can calculate from (4.19) and (4.38) that

$$
\frac{\alpha_{C I A}\left(N_{2}-N_{2}\right) 0.79^{2}}{\alpha_{C I A}^{M P M}} \approx 1.17,
$$

quite different from the usual assumption ${ }^{27,} 79$ that MPM uses the nitrogen CIA, scaled by $0.79^{2}$, to describe the dry air CIA.

The more detailed analysis of the absorption contributions from different physical origins and therefore different temperature and frequency dependencies requires broadening of the frequency range and further improvements of the measurement accuracy. The total dry air absorption, the quantity of interest for the atmospheric applications, was measured in the $170-260 \mathrm{GHz}$ range for the number of temperatures and pressures and is well described by equation (4.37).

\subsubsection{Moist air absorption}

Moist air is prepared by first filling the evacuated resonator with water vapor by connecting the chamber to a container with demineralized distilled water. The appropriate amounts of nitrogen and oxygen of the same quality as used for the dry air measurements are added after the required water vapor pressure is achieved. This allows the absorption to be studied as a function of a full set of parameters: frequency, temperature, and the partial pressures of nitrogen, oxygen and water. 
Room temperature moist air absorption for a number of different water partial pressures is shown in Figure 4.26 along with prediction of MPM89. Dry air absorption is shown on the same plot to demonstrate the relative size of the effects. Our broad frequency scan covers both the $183 \mathrm{GHz}$ water line and the region far from the line center, where the $183 \mathrm{GHz}$ line contribution to the total absorption is small and continuum absorption dominates.

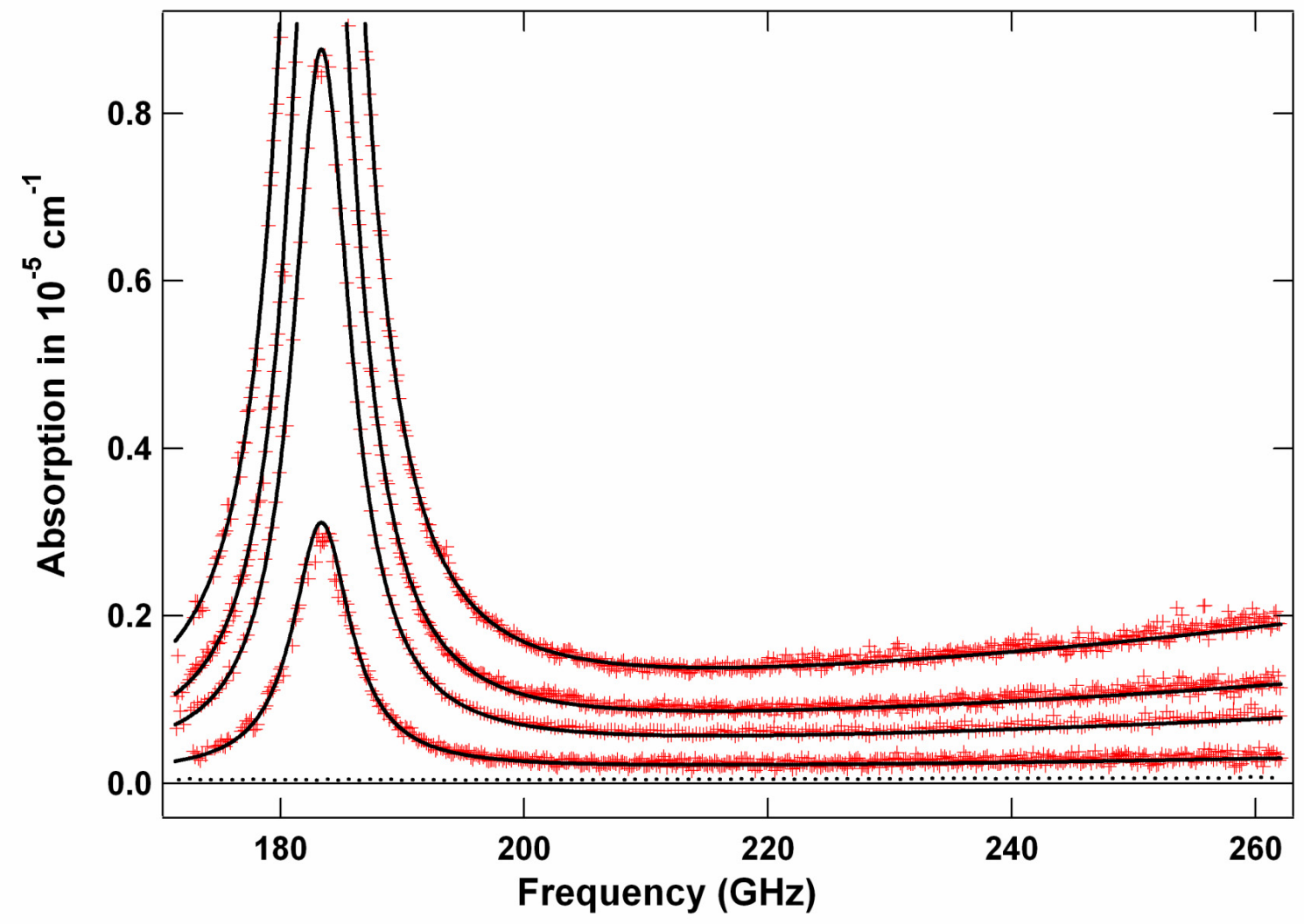

Figure 4.26: The 20 point averaged room temperature moist air absorption for the 760 Torr total pressure and $0.35,1.0,1.5$ and 2.4 Torr of water partial pressure $(+)$. Solid lines are predictions of the MPM89. 760 Torr dry air absorption $(\bullet)$ is shown to demonstrate the relative size of the effect.

For each set of the system parameters, the absorption is first fit to a Van Vleck-Weisskopf line profile with an addition of a constant, linear and a quadratic frequency terms to account for the moist air continuum and the higher frequency water lines contributions. The line center, the line width and the peak absorption are 
free varying parameters of the fit. From the line profile fit, the amount of water gas in the resonator can be found by using the line parameters from the JPL catalog ${ }^{17}$ and equations (2.12) and (2.10). The $183 \mathrm{GHz}$ line absorption is then subtracted from the total measured moist air absorption and the remaining absorption is studied as a function of the system parameters. The definition of "continuum" is impossible without explicitly stating what was used to account for the line contributions. Factors including uncertainties in the line parameters, especially their temperature dependencies, questionable validity of the standard line-shape theory at the far wings of the absorption lines, similar frequency dependence of the "line contributions" and the "excess continuum absorption" at our frequency range, result in somewhat unwarranted differentiation between what is considered to be the "continuum" and what is attributed to the far wings of the higher frequency lines absorption. Instead of choosing one particular set of water line parameters and line shapes, from now on, unless otherwise noted, we will call what remains after the $183 \mathrm{GHz}$ line subtraction the "moist air continuum" and study its behavior as a function of the gas partial pressures, frequency and temperature. To compare our results with the literature, it is important to carefully use the chosen by a particular group parameterization for the water line contributions.

Because our room temperature 1 atm absorption was well described by the Liebe's MPM 89, as seen in Figure 4.26, we first attempted to use a simple frequency squared dependence to describe our moist air continuum absorption. Figure 4.27 shows the 1 atm room temperature moist air absorption for 1 Torr of water partial pressure along with a fit to a Van Vleck-Weisscopf lineshape for a $183 \mathrm{GHz}$ line and a frequency squared term for the moist continuum absorption as 


$$
\alpha_{\text {MoistAir }}^{P_{D_{r y}}=759 \text { Torr, } P_{\mathrm{H}_{2} \mathrm{O}}=1 \text { Torr, }{ }^{2}=24 C}(v)=\alpha_{V V W}^{183}(v)+A_{\text {MoistCont }} v^{2},
$$

where

$$
\begin{gathered}
P_{\text {Dry }}=P_{\text {Total }}-P_{\mathrm{H}_{2} \mathrm{O}}, \\
\alpha_{V V W}^{183}(v)=C_{1} v^{2}\left(\frac{1}{\left(v-C_{2}\right)^{2}+C_{3}{ }^{2}}+\frac{1}{\left(v+C_{2}\right)^{2}+C_{3}{ }^{2}}\right),
\end{gathered}
$$

and $A_{\text {MoistCont }}, C_{1}, C_{2}$, and $C_{3}$ are free fitting coefficients, corresponding to the moist continuum coefficient $\left(A_{\text {MoistCont }}\right)$, the line peak intensity $\left(\frac{C_{1} v^{2}}{C_{3}{ }^{2}}\right)$, the line center $\left(C_{2}\right)$ and the pressure broadened line width $\left(C_{3}\right)$, respectively.

Although the far wings absorption does not behave with frequency exactly as the frequency squared (approximation valid when the measurement frequency is much smaller than the line center frequency), the relative size of the far wings contribution and its frequency dependence makes it possible to describe the moist air continuum absorption, as defined above, by a simple frequency squared dependence within the experimental errors, as evident from the fit in Figure 4.27.

To make sure that the deviation of the far wings absorption from the frequency squared dependence does not affect the measured continuum coefficient, we have compared the results of the fits for two different cases. In the first case, the absorption as a function of frequency was fit to the Van Vleck-Weisscopf lineshape function for the $183 \mathrm{GHz}$ line with an addition of the constant, linear and quadratic in frequency terms. The $183 \mathrm{GHz}$ contribution was then subtracted and the result was fit with a frequency squared function to obtain the $A_{\text {MoistCont }}$. In the second case, the absorption was fit with equation (4.43) with four free fitting parameters. The resulting $A_{\text {MoistCont }}$ 
for the two cases were the same within the fit uncertainty. This means that in our measurement frequency range the moist air continuum absorption is well described by a simple frequency squared behavior and evaluation of the $A_{\text {MoistCont }}$ is readily translated into the full moist air absorption at any frequency by the use of equation (4.43).

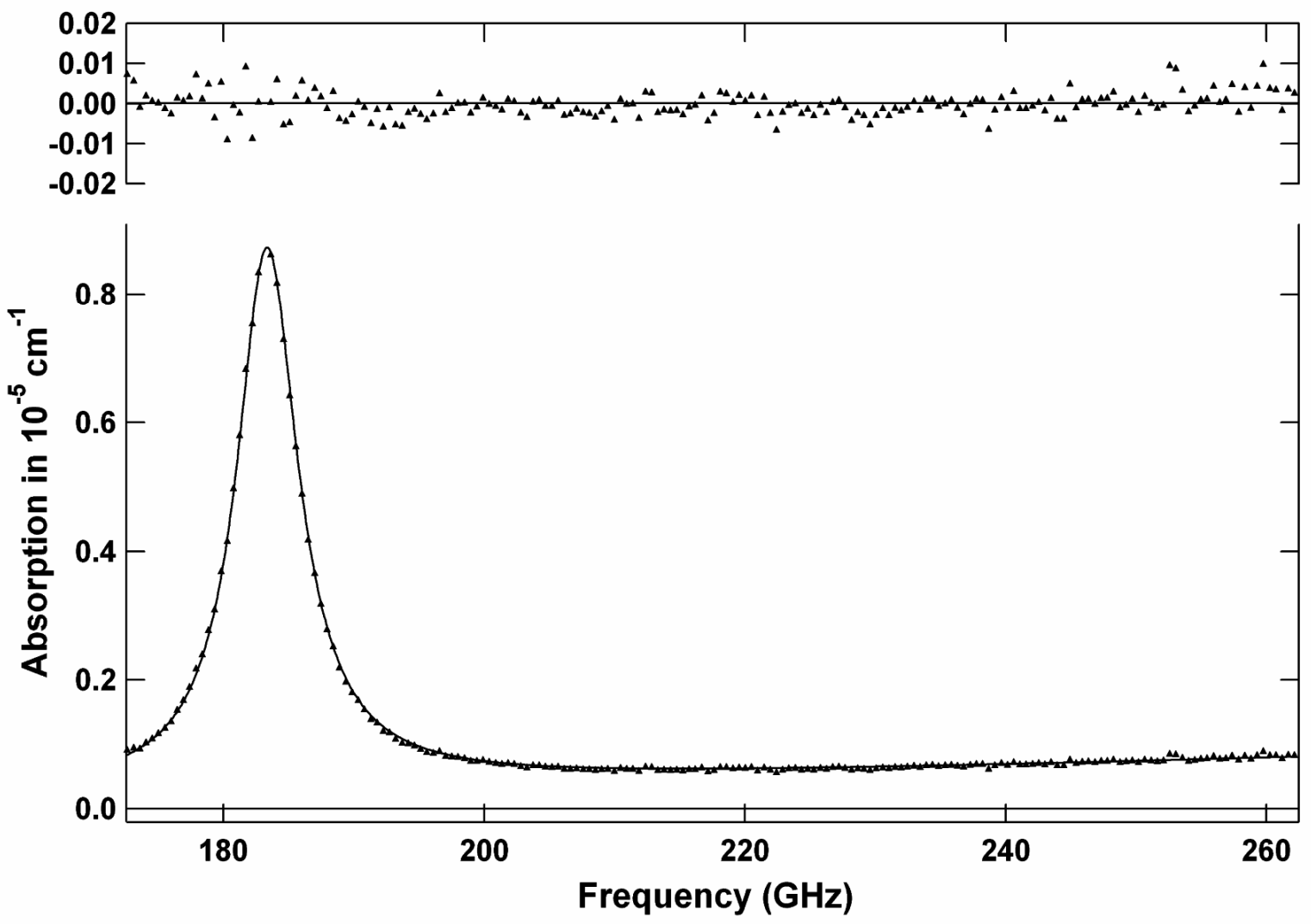

Figure 4.27: 30 point average of the moist air absorption for 1 atm total pressure and 1 Torr of water vapor pressure. Solid line is a fit to a Van VleckWeisscopf line profile and a frequency squared term. The residual of the fit is shown at the top of the figure on an expanded scale.

To study the moist continuum pressure dependence, we can start with the standard parameterization of the moist air continuum absorption with three terms corresponding to the water-water, dry air-dry air, and water-dry air collisions as

$$
\begin{aligned}
& A_{\text {MoistCont }}\left(P_{\text {Dry },} P_{\mathrm{H}_{2} \mathrm{O}}\right)=A_{\text {Self }}+A_{\text {Foreign }}+A_{\text {Dry }}= \\
& =C_{\text {Self }} P_{\mathrm{H}_{2} \mathrm{O}}{ }^{2}+C_{\text {Foreign }} P_{\mathrm{H}_{2} \mathrm{O}} P_{\text {Dry }}+C_{\text {Dry }} P_{\text {Dry }}{ }^{2}
\end{aligned}
$$


For all our room temperature measurements, the contributions from the self continuum and from the dry air continuum are both small compared with the foreign continuum. We have used our measurements of the dry air absorption and Liebe MPM $89^{28}$ self continuum absorption to subtract from the total moist continuum to study the behavior of the moist air foreign continuum.

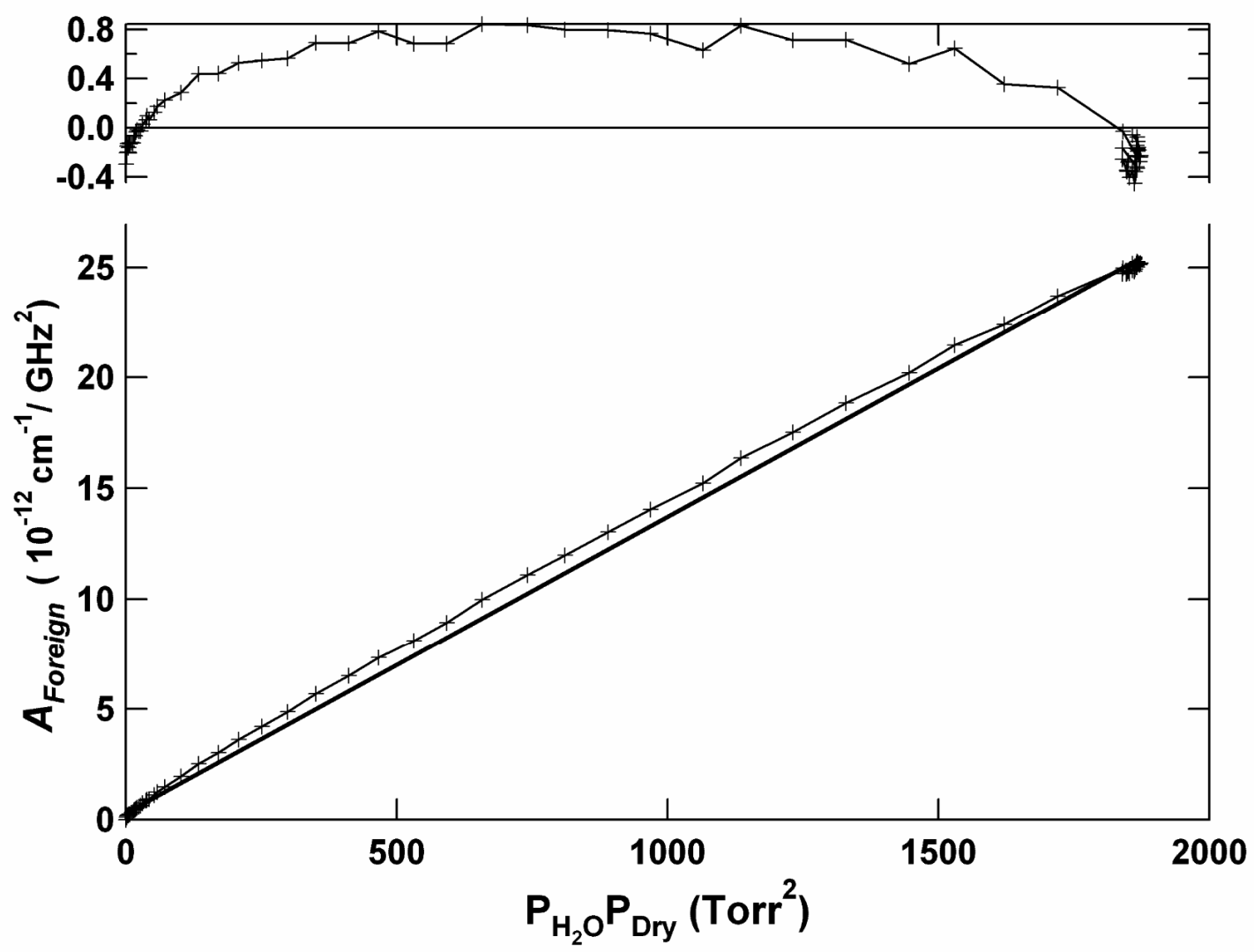

Figure 4.28: The moist air foreign continuum $A_{\text {Foreign }}$ vs. the product of the dry air and water pressures. The starting total pressure is 760 Torr, the starting water vapor pressure is 2.4 Torr. The residual of the fit to a straight line is shown at the top of the figure on an expanded scale. 
Figure 4.28 shows how the foreign moist continuum absorption, calculated as described above, varies as a function of the product of the dry air pressure and the water pressure as the chamber is pumped out from the starting 760 Torr of total pressure and 2.4 Torr of water vapor partial pressure. As can be seen from Figure 4.28 , the dependence is close to, but not exactly, linear. To make sure this is not a non equilibrium effect related to pumping on the chamber, we have checked that the effect does not vanish for different pump down speeds from one and a half to four hours to get from 1 atm to under 1 Torr, stopping and waiting while taking data, as well as varying the starting total pressure. We have also explicitly checked that for the MPM89 parameterization of the water lines absorption, the far wings contribution varies exactly as a product of the dry air pressure times the water pressure and therefore can not be accountable for the described above effect without modifying the line shape function use by the MPM. From the comparison of our absorption data for a number of starting water pressures it was also clear that the possible error in the MPM89 value of the moist air self continuum can not explain the above nonlinearity. 


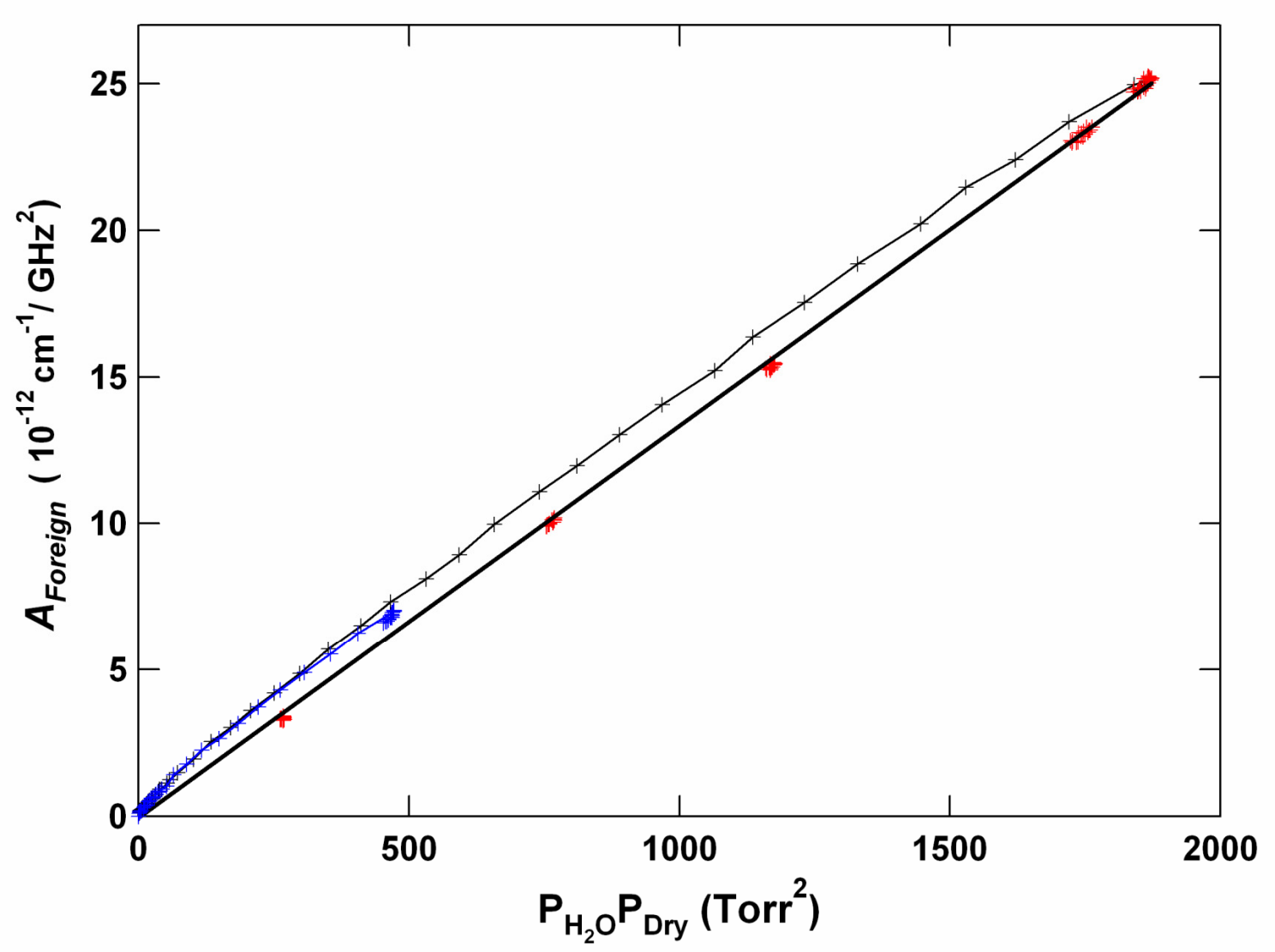

Figure 4.29: The moist air foreign continuum coefficient $A_{\text {Foreign }}$ vs. the product of the dry air and water pressures. 760 Torr total pressure, $0.35,1.0$, 1.5, 1.9, and 2.4 Torr of water (red). The pump down from the starting total pressure of 760 Torr and water vapor pressure of 2.43 Torr (black). The pump down from the starting total pressure of 380 Torr and the water vapor pressure of 1.2 Torr (blue).

Further analysis showed that if the total dry air pressure was kept constant while the water fraction varied, the moist air foreign continuum increased close to linearly with the water pressure. For example, although for each day the $A_{\text {Foreign }}$ results for the pump down are not linear with the product of water and dry air pressures, the 1atm results are very close to linear, as demonstrated in Figure 4.29. This behavior implies that the moist air foreign continuum has the following functional dependence on the dry air and water pressures 


$$
A_{\text {Foreign }}\left(P_{\text {Dry },} P_{\mathrm{H}_{2} \mathrm{O}}\right)=F\left(P_{\text {Dry }}\right) P_{\mathrm{H}_{2} \mathrm{O}}
$$

where $F\left(P_{D r y}\right)$ could be any function. The simplest one to try is a third order polynomial of the form

$$
F\left(P_{D r y}\right)=A+B P_{D r y}+C P_{D r y}^{2},
$$

with $\mathrm{B}$ corresponding to the expected linear behavior, $\mathrm{C}$ is possibly due to a three body collision of two dry air molecules with the water molecule, and A with no clear spectroscopic meaning.

Results for the $F\left(P_{D r y}\right)$, obtained by dividing $A_{\text {Foreign }}\left(P_{D r y}, P_{\mathrm{H}_{2} \mathrm{O}}\right)$ by the water pressure for one of the starting humidities are shown in Figure 4.30. The behavior of the $F\left(P_{D r y}\right)$ is well described by the first two terms of (4.48). We can combine equations (4.46)-(4.48) to obtain a final result for the measured moist air continuum in the form

$$
\begin{aligned}
& A_{\text {MoistCont }}\left(P_{\text {Dry }}, P_{\mathrm{H}_{2} \mathrm{O}}\right)=A_{\text {Self }}+A_{\text {Foreign }}^{W L}+A_{W L}+A_{\text {Dry }}= \\
& =C_{\text {Self }} P_{\mathrm{H}_{2} \mathrm{O}}^{2}+C_{\text {Foreign }}^{W L} P_{\mathrm{H}_{2} \mathrm{O}} P_{\text {Dry }}+C_{W L} P_{\mathrm{H}_{2} \mathrm{O}}+C_{\text {Dry }} P_{\text {Dry }}^{2}
\end{aligned}
$$

where $C_{W L}$ represents a linear with water pressure term $A$ from (4.48). 


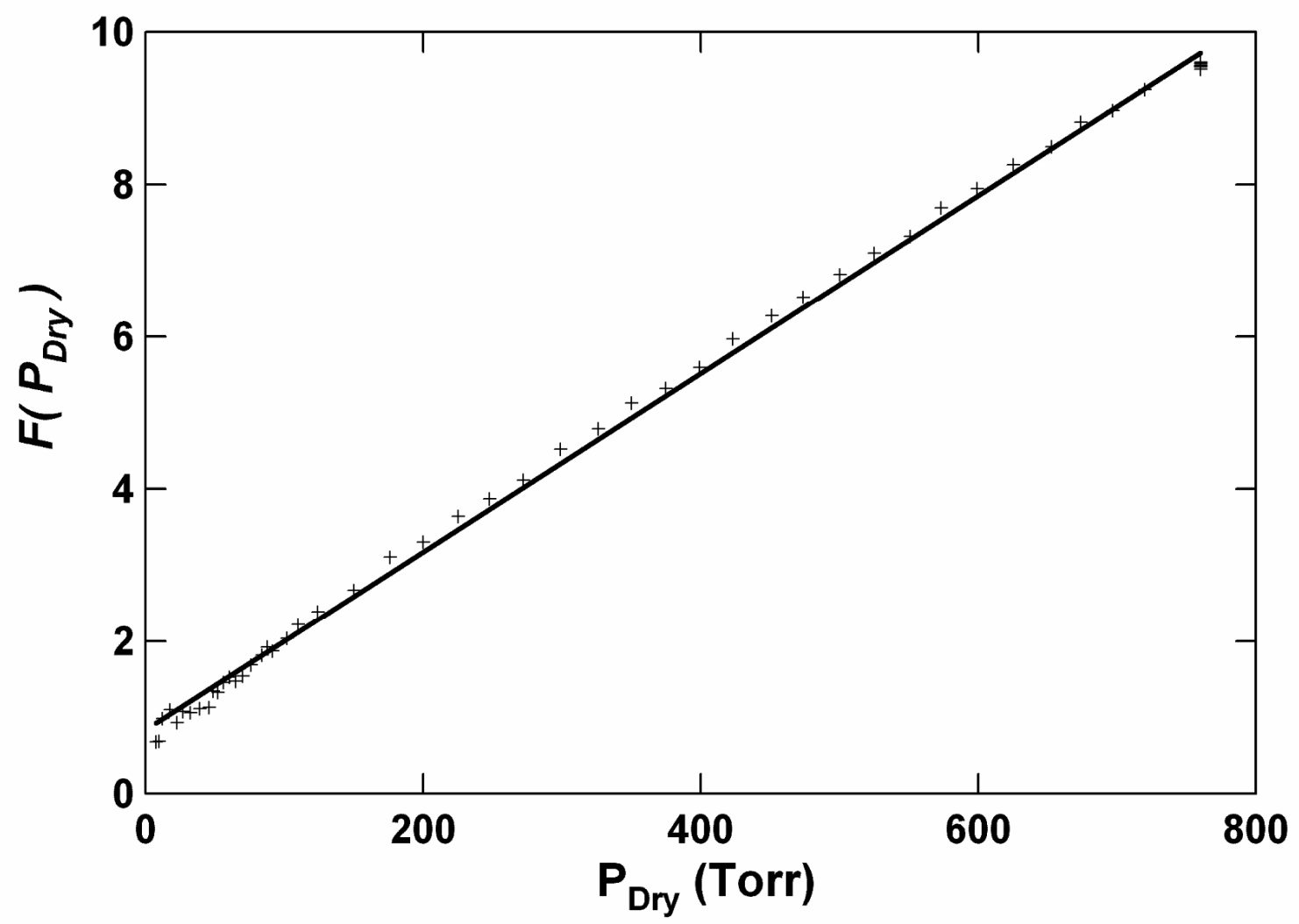

Figure 4.30: The $F\left(P_{D r y}\right)$ dependence on the dry air pressure for 760 Torr total and 2.4 Torr water starting pressures. Solid line is a linear fit of the data.

To check the pressure behavior of the moist air continuum absorption, we have measured the absorption of pure water vapor. The frequency dependence was once again well described by a simple frequency squared law, with equation (4.49) for the case of pure water becoming

$$
A_{\text {MoistCont }}\left(P_{\text {Dry }}=0, P_{\mathrm{H}_{2} \mathrm{O}}\right)=C_{\text {Self }} P_{\mathrm{H}_{2} \mathrm{O}}{ }^{2}+C_{W L} P_{\mathrm{H}_{2} \mathrm{O}}
$$

Figure 4.30 shows the pure water continuum, divided by the water pressure, dependence on the pressure. A linearly increasing with the water pressure term $C_{W L}$ is clearly present and is manifested by a non zero $\mathrm{Y}$-axes intersection. 


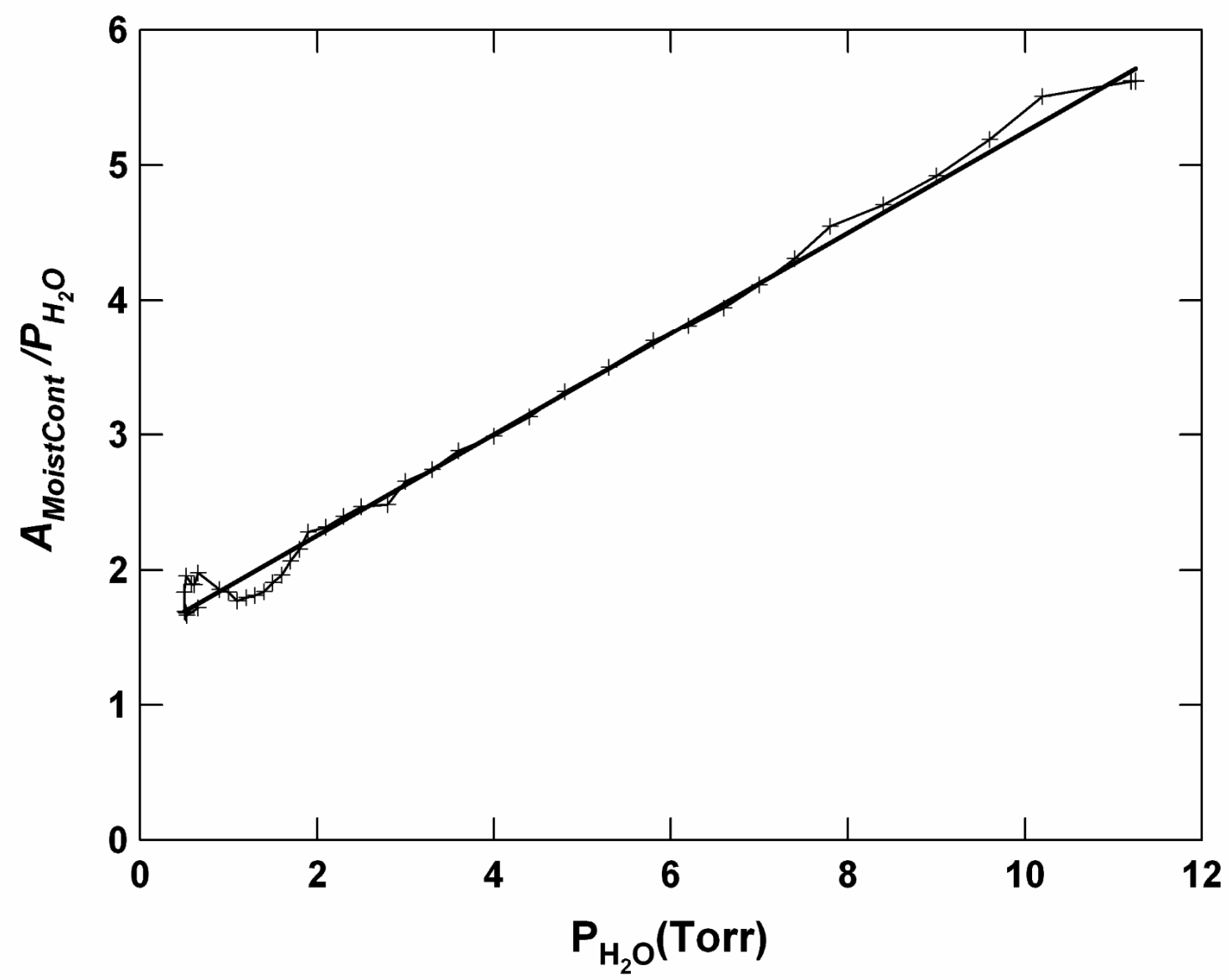

Figure 4.31: Pure water continuum coefficient $A_{\text {MoistCont }}$, divided by the water pressure, as a function of the water pressure.

Before we proceed to the speculation on whether this linear in water term has the gas absorption origin or it is purely a systematic effect, we will first present our measurements of the moist air absorption temperature dependence.

Most field studies of the moist air absorption in the microwave are performed at atmospheric pressures. It therefore makes sense to start the temperature dependence study by comparing our measurements performed at different temperatures but for the same total pressure of 760 Torr. Figure 4.32 shows the moist air foreign continuum coefficient $A_{\text {Foreign }}$ from equation (4.46), calculated by subtracting the dry and water self continua from the full moist air continuum, for a number of temperatures and 
water pressures, all measured at the same 760 Torr of the total pressure. One can see that for each temperature the dependence of the $A_{\text {Foreign }}$ on the water pressure is linear within the experimental uncertainty.

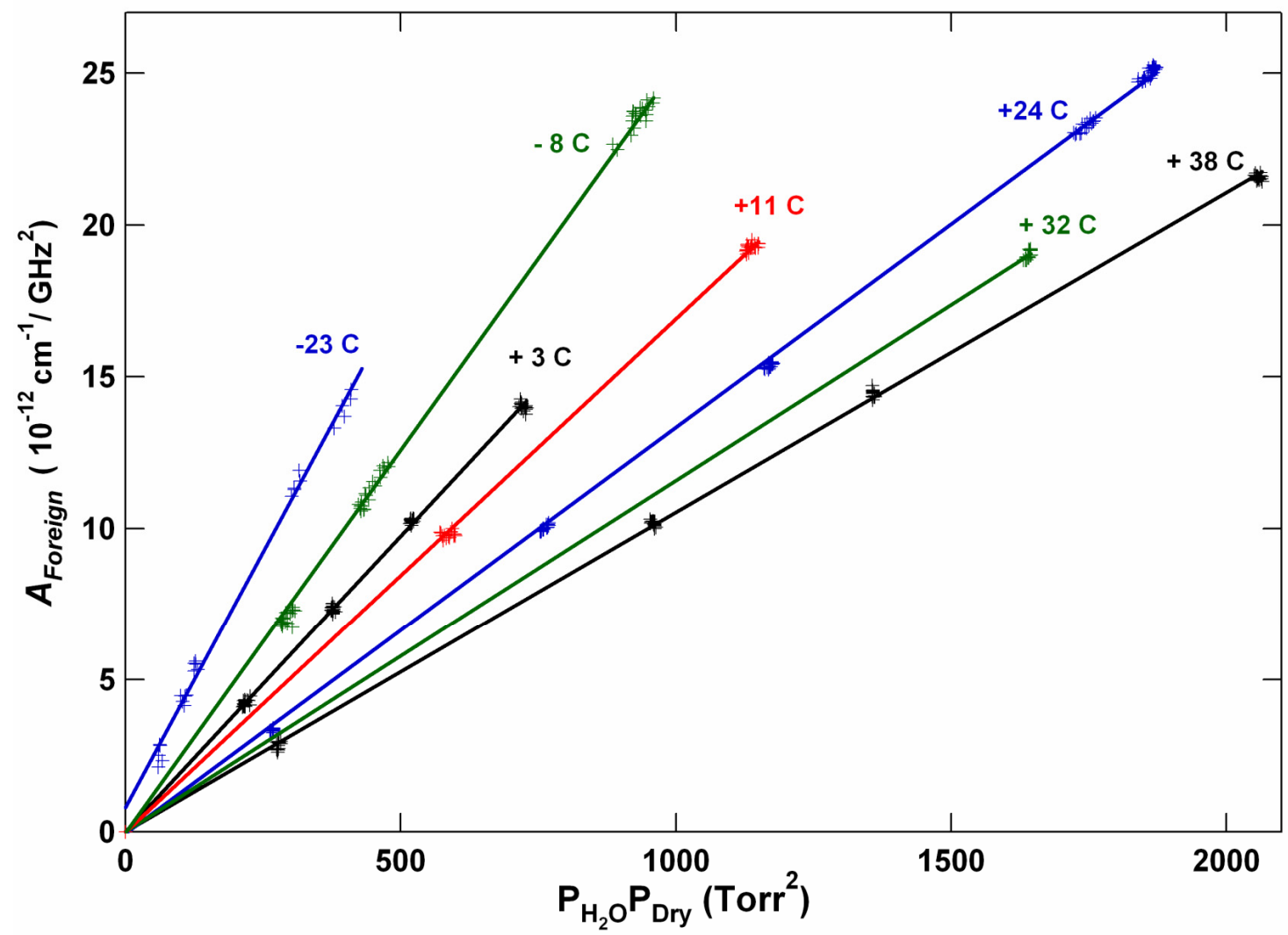

Figure 4.32: 1 atm moist air foreign continuum absorption coefficient $A_{\text {Foreign }}$ for a number of temperatures and water pressures.

The $-23{ }^{\mathrm{O}} \mathrm{C}$ data is a noticeable exception, as the best fit line does not have the zero interception. Although not significant for the $-8{ }^{\circ} \mathrm{C}$ measurements, it seems that there is another effect, introducing the extra loss due to the ice forming on the mirrors for the temperatures below freezing. This effect was shown in Figure 4.4 in the section 4.2 .3 for the $-23{ }^{\circ} \mathrm{C}$ data. Another demonstration of the ice effect is 
$-43{ }^{\mathrm{O}} \mathrm{C}$ continuum absorption data, shown in Figure 4.33. The non-zero interception of the best fit straight line is consistent with ice staying on the mirrors through the pump down until the low pressure below 1 Torr is reached.

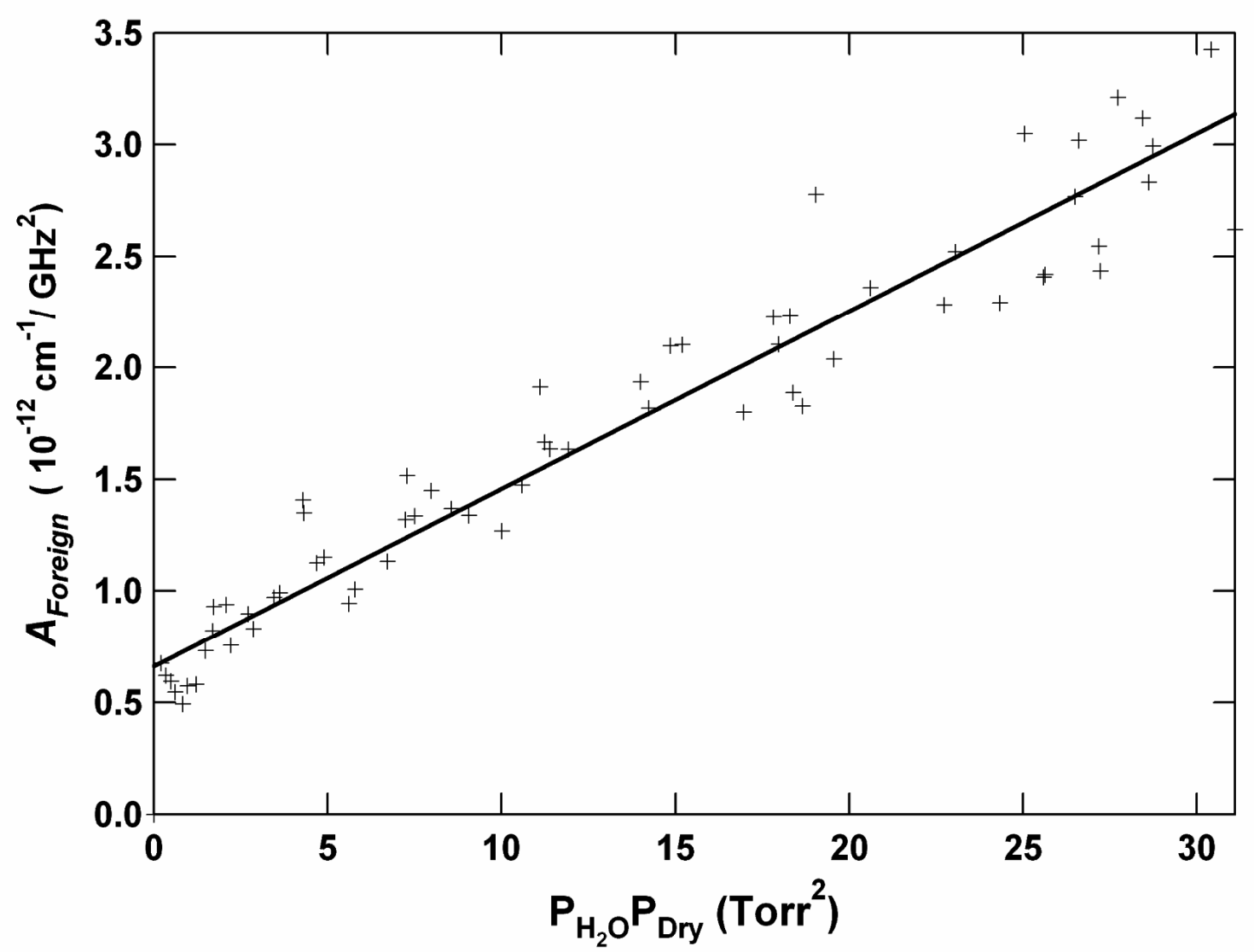

Figure 4.33: $-43{ }^{\mathrm{O}} \mathrm{C}$ moist air foreign continuum coefficient $A_{\text {Foreign }}$ vs. the product of the dry air and water pressures.

At each of the temperatures above $0{ }^{\mathrm{O}} \mathrm{C}$ and for the $-8{ }^{\mathrm{O}} \mathrm{C}$ the $C_{\text {Foreign }}$ was calculated as a slope in the straight line best fit with fixed zero intercept. For the $-43{ }^{\mathrm{O}} \mathrm{C}$ measurements, the slope of the linear fit shown in Figure 4.33 was used. For the $-23{ }^{\mathrm{O}} \mathrm{C}$ measurements the $1 \mathrm{~atm}$ data for five different water pressures was fit to a straight line with a zero intercept as a free variable parameter. The results of the moist air foreign continuum coefficient measurements for a number of temperatures are presented in Table 4.5 and Figure 4.34. 


$\begin{array}{cc}\text { Temperature }(\mathrm{K}) & C_{\text {Foreign }}(T)\left(10^{-15} \mathrm{~cm}^{-1} /\left(\mathrm{Torr}^{2} \mathrm{GHz}^{2}\right)\right) \\ 230.0 & 52.0(26) \\ 252.2 & 33.7(17) \\ 265.2 & 25.2(12) \\ 276.5 & 19.5(10) \\ 284.2 & 16.9(8) \\ 297.2 & 13.1(7) \\ 305.0 & 11.6(6) \\ 311.2 & 10.5(5)\end{array}$

Table 4.5: Moist air foreign continuum coefficient $C_{\text {Foreign }}$ temperature dependence. Uncertainty is estimated at $5 \%$. 


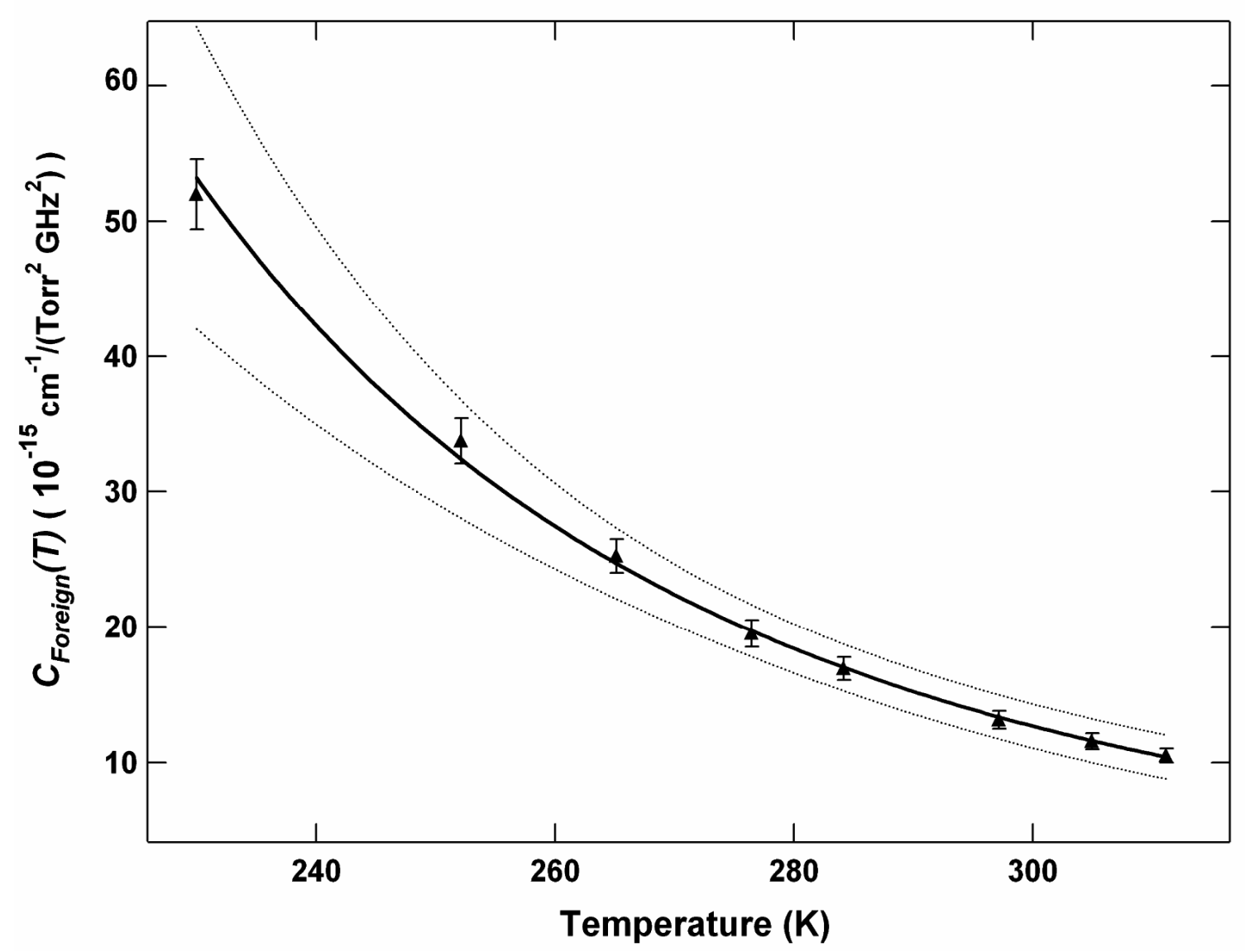

Figure 4.34: The temperature dependence of the moist air foreign continuum coefficient $C_{\text {Foreign }}$, measured at $1 \mathrm{~atm}$. Solid line is a fit to a power law. Dotted lines are the $3 \sigma$ confidence bands.

The temperature dependence of the moist air foreign continuum coefficient was fit to a power law, resulting in

$$
C_{\text {Foreign }}(T)=13.0(2) 10^{-15}\left(\frac{T}{300}\right)^{-5.24(15)} \mathrm{cm}^{-1} /\left(\operatorname{Torr}^{2} \mathrm{GHz}^{2}\right)
$$

This is a result of the fit of our 760 Torr measurements only.

We will now proceed to the measurements made at all the pressures to study the nonlinear effect, exhibited for pressures under $1 \mathrm{~atm}$. For each room temperature pump down, the moist continuum absorption was fit to equation (4.49) with $C_{F o r e i g n}^{W L}$ and $C_{W L}$ as free varying parameters, $C_{D r y}$ from our dry air measurements and 
$C_{\text {Self }}$ from MPM89. As shown in Figure 4.35 for a fit of the pump down results for the starting water pressure of 2.4 Torr, inclusion of the linear with water pressure term does reduce the residuals of the fit to a straight line, although there still seems to be some systematic deviation remaining.

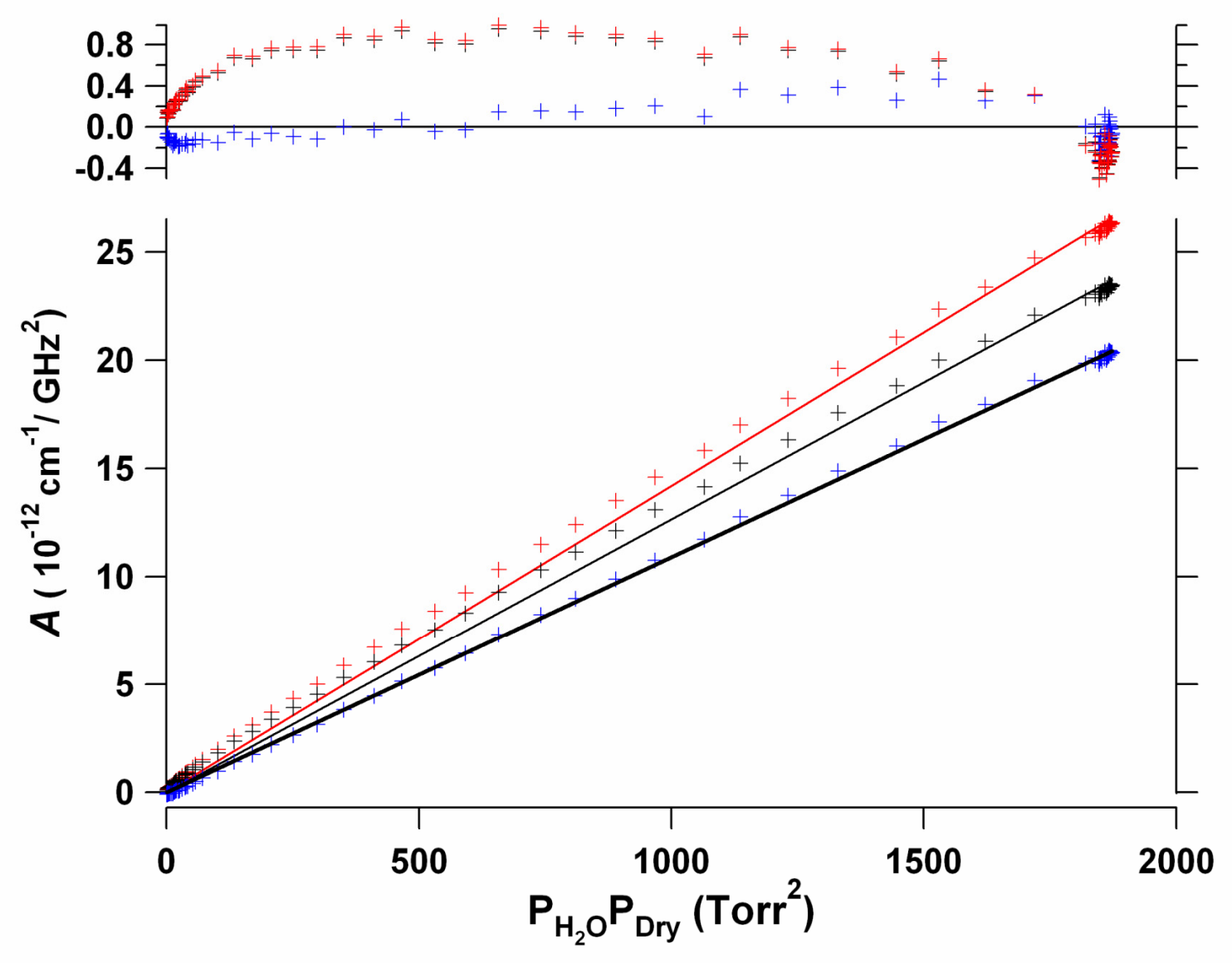

Figure 4.35: Moist air continuum coefficient as a function of the product of the dry air and water pressures. Starting water pressure is 2.4 Torr. Starting total pressure is 760 Torr. Full moist continuum $A_{\text {MoistCont }}$ (red), foreign continuum $A_{\text {Foreign }}$ (black), calculated by subtracting the water self continuum and the dry air continuum, and the foreign continuum $A_{\text {Foreign }}^{W L}$ (blue), calculated by further subtracting the linear with water term $A_{W L}$ from the fit with equation (4.49) is shown. Respective residuals of the fit to a straight line are shown at the top of the figure on an expanded scale. 
Figure 4.36 shows how the coefficients $C_{F o r e i g n}^{W L}$ and $C_{W L}$ vary for different water pressures. It also shows the $C_{\text {Foreign }}$ coefficient, calculated for the respective $1 \mathrm{~atm}$ measurements, and the $C_{W L}$ from the pure water measurement. The linear water term coefficient $C_{W L}$ is slightly increasing with the increasing starting water pressure, while both $C_{F o r e i g n}^{W L}$ from the 4.49 fit and $C_{\text {Foreign }}$ from 1 atm measurements stay constant.
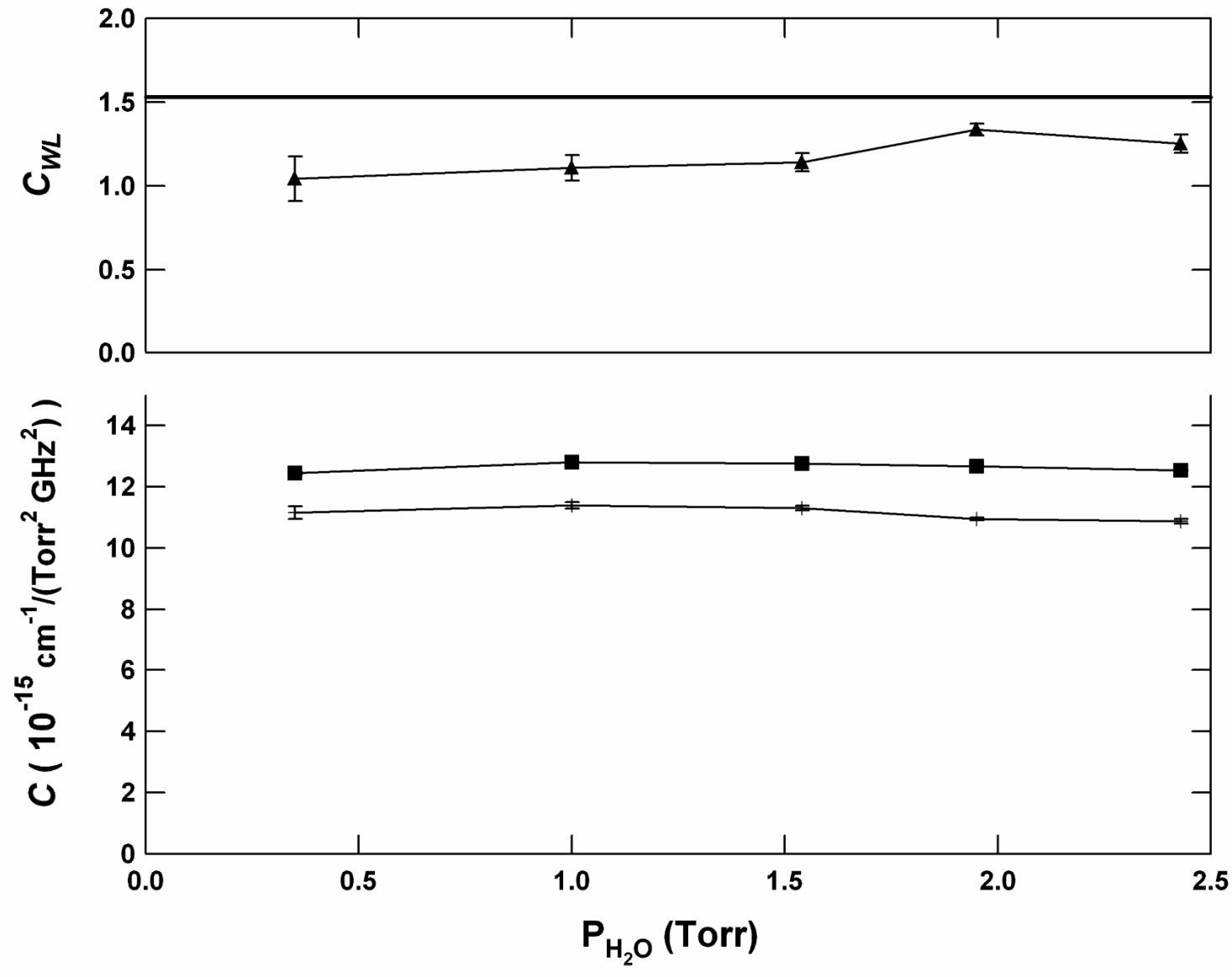

Figure 4.36: Moist air absorption continuum coefficients $C_{W L}(\mathbf{\Delta}), C_{F o r e i g n}^{W L}(+)$, and $C_{\text {Foreign }}(\mathbf{-})$ for the five starting water pressures from 0.35 to 2.4 Torr. $C_{W L}$, deduced from the pure water measurements, is shown as a straight line on the top plot. 
The same fit was performed for all our measurements at different temperatures with results for $C_{\text {Foreign }}^{W L}$ presented in Figure 4.37 along with the discussed above 1 atm $C_{\text {Foreign }}$ measurements and the MPM 89 predictions. High uncertainties of the $C_{\text {Foreign }}^{W L}$ for $-23 \mathrm{C}$ and $-43 \mathrm{C}$ data indicate difficulties in separating the linear with water pressure term from the total moist continuum absorption as the temperature gets lower and the maximum amount of water in the chamber gets limited by the saturation vapor pressure. The difference in the absolute value and the temperature dependence of the $C_{F o r e i g n}^{W L}$ and $C_{\text {Foreign }}$ indicates that the origin of the linear with water term $C_{W L}$ needs to be better understood before a fruitful comparison with theory of other groups experimental results is to be performed.

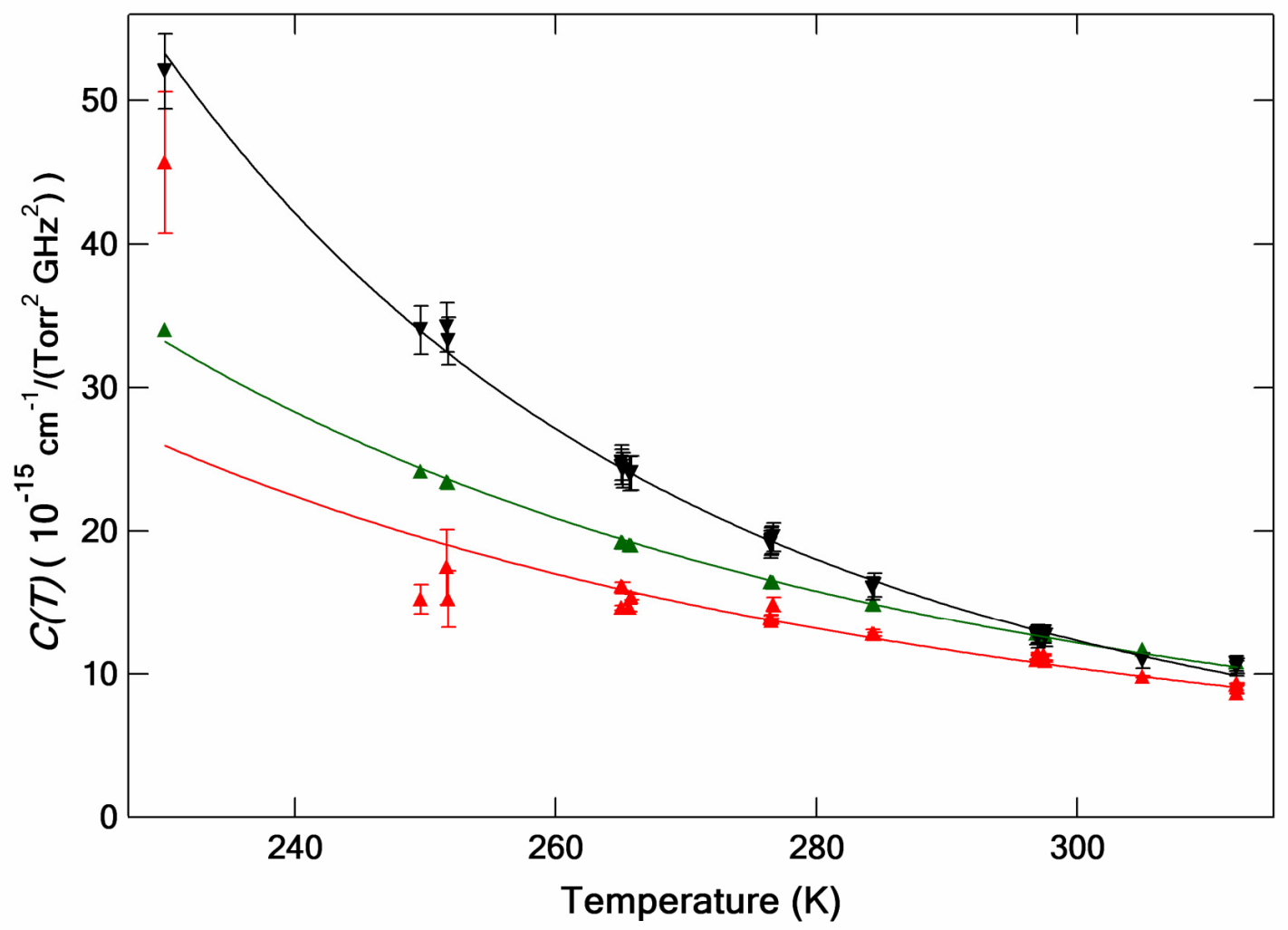

Figure 4.37: The temperature dependence of the foreign moist air continuum coefficient $C_{\text {Foreign }}^{W L}($ red), 1atm moist air foreign continuum absorption coefficient $C_{\text {Foreign }}$ (black) and the MPM 89 prediction (green). 
As was discussed above in the oxygen and dry air sections, when two or more effects with different pressure and frequency dependences combine to give the final absorption, uncertainties in estimating each of the contributions can be much greater than the uncertainty of the actual measurement. We have to keep this in mind when we separate the linear in water pressure term from the total absorption. If the linear with water effect discussed above has a gas phase absorption origin or even if it is related to an unknown error in processing of the sub-atmospheric pressure measurements, then the above analysis of the 1 atm absorption data presents a valid, albeit not full, study of the moist air absorption and its temperature dependence. On the other hand, if this effect is systematic, whether due to changes in the mirror reflectivity, liquid water absorption or the beam splitter coupling changes; than the actual gas phase measurement accuracy is limited by the ability to separate the linear term from the product term, not by the precision of the resonator loss measurements. In this case, as can be seen from equation (4.48), the relative size of this effect gets smaller as the dry air pressure increases, making the 1 atm measurements closest to the gas phase absorption and justifying our separate study of the 760 Torr measurements described above.

There is a straightforward way to separate the gas phase absorption from the rest of the resonator losses on a hardware level. It requires an addition of a third mirror to the system setup as described below and is planned to be implemented in the nearest future. 


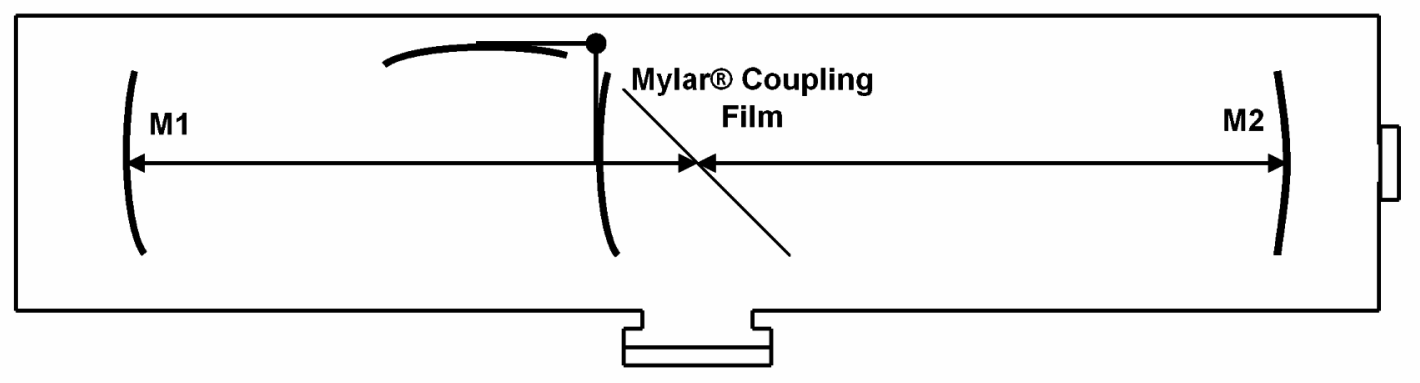

Figure 4.38: An addition of the third mirror to aid in separating the gas phase absorption from the rest of the resonator losses.

The gas phase absorption is the only resonator loss that changes linearly with the mirror separation. The mirror losses and the beam splitter losses remain exactly the same. If the resonance mode intensity distribution is not dramatically changed, the gas absorption is the only loss that changes at all, as the diffraction loss and the output coupling loss too remain constant. Although it would be very difficult to implement an experimental setup where one of the mirrors could move a considerable distance with respect to the initial mirror separation without losing the alignment; there is a simple, more straightforward way to achieve the same desired result. We plan to put a retractable mirror close to the center of the resonator chamber, as shown in Figure 4.38. This mirror will be flat, which will essentially keep the resonator mode structure the same. By comparing the resonator loss with and without the third mirror, it will be possible to separate the gas phase losses from the rest of the losses of the resonator in a straightforward manner. This should give us more insight on the origin of the moist air foreign continuum absorption deviation from the linear dependence on the product of the water and dry air pressures. 


\subsection{310 GHz WATER LINE PRESSURE BROADENING AND PRESSURE SHIFT MEASUREMENTS}

Atmospheric absorption of water around the $183.310 \mathrm{GHz}$ absorption line is very important in the atmospheric retrieval. The ability of our instrument to make absorption measurements covering the full $183 \mathrm{GHz}$ line profile for a wide range of pressures allows us to study this line in detail.

As was described in the previous section, for each set of the system parameters we have fitted the moist air absorption to the Van Vleck - Weisskopf line profile with an addition of a constant, linear and a quadratic in frequency terms to account for the moist air continuum absorption. The line center frequency and the line width were free varying parameters in the fit. The frequency calibration procedure for a filled resonator was described in Section 4.3. For each temperature and water starting partial pressure, the measured line shift and the line width dependence on the total air pressure was fitted to a straight line. Figures 4.39 and 4.40 show the room temperature measurements of the line width and the line shift as a function of pressure. 

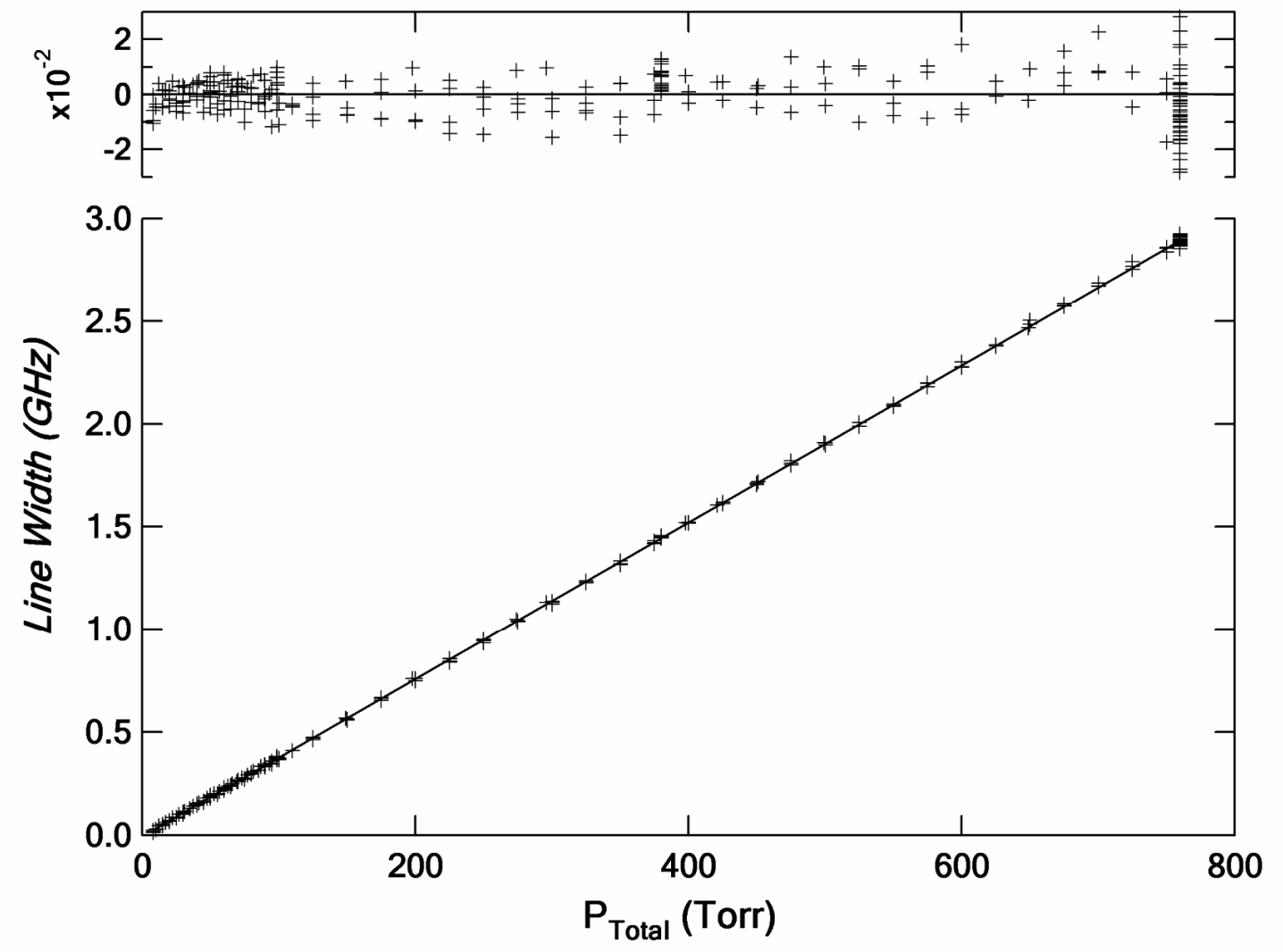

Figure 4.39: Room temperature $183 \mathrm{GHz}$ water line width as a function of the total pressure for four water starting pressures from 0.35 to 2.4 Torr. Solid line is a linear fit of the data. Residuals of the fit are shown at the top of the figure on an expanded scale.

As expected, the pressure broadened line width increased linearly with pressure as

$$
\Delta v=\gamma_{B}(T) P_{A i r},
$$

where $\Delta v$ is the $183 \mathrm{GHz}$ water line width, $\gamma_{B}(T)$ is the air pressure broadening parameter, $P_{\text {Air }}$ is the total air pressure. The measured line shift pressure dependence was also fitted to a linear function as

$$
\delta v=v(P)-v(0)=\gamma_{S}(T) P_{A i r},
$$

where $\delta v$ is the line center shift from the low pressure value, $\gamma_{S}(T)$ is the air line center pressure shift parameter. 


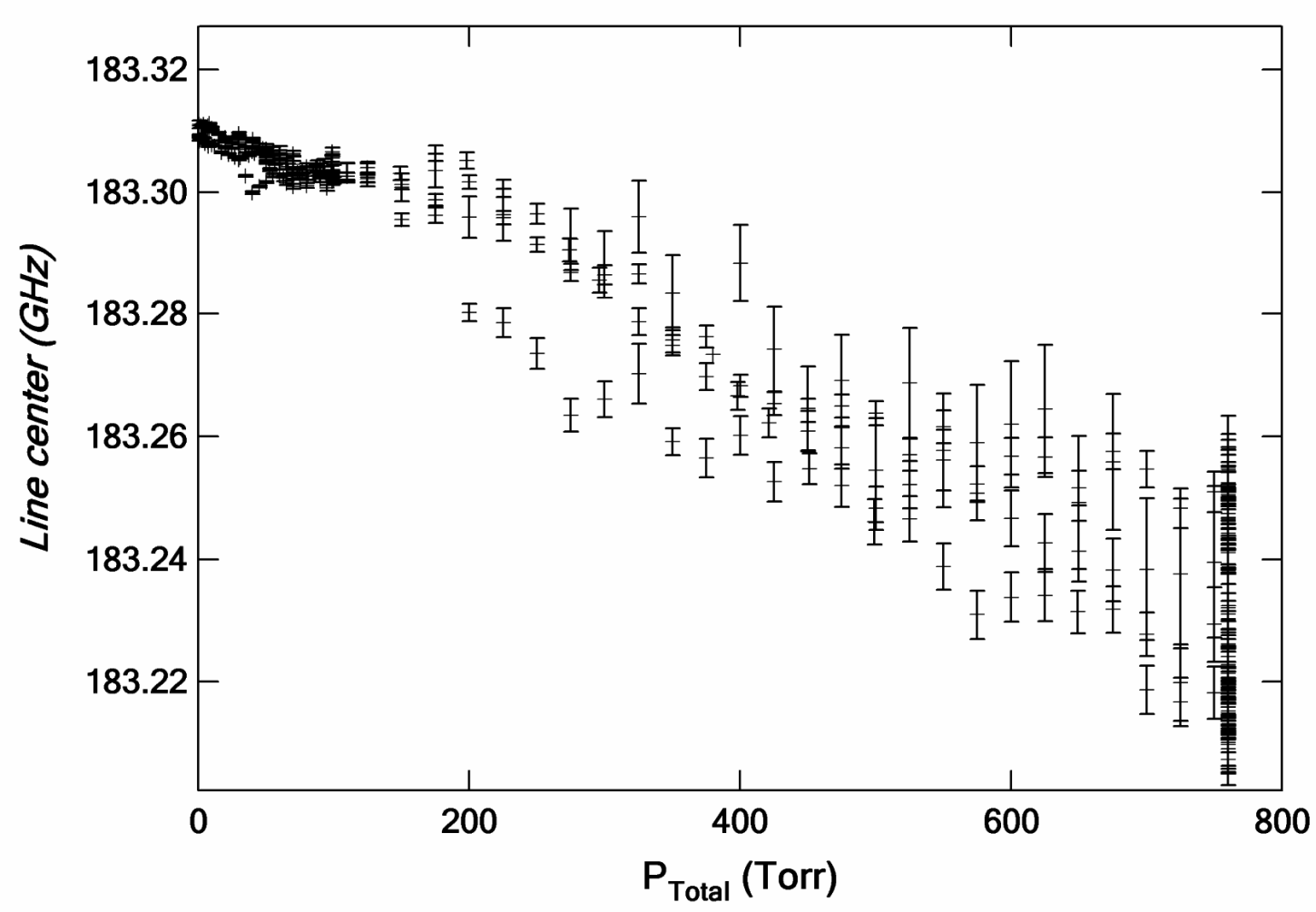

Figure 4.40: Room temperature $183 \mathrm{GHz}$ water line center frequency as a function of the total pressure for four water starting pressures from 0.35 to 2.4 Torr.

Results of the measurements at a number of temperatures in the $-40{ }^{\circ} \mathrm{C}$ to $+60{ }^{\circ} \mathrm{C}$ range are summarized in Table 4.6. For each temperature the results of measurements with varying water partial pressures were averaged together to obtain the final value. The pressure broadening coefficient uncertainty is due to the pressure and temperature uncertainty and is estimated at $0.5 \%$. The accurate measurement of the line shift is very difficult because the value of the shift is small compared with the line width and systematic effects, mainly baseline subtraction related, could be several times greater than the statistical uncertainty of a single measurement. The uncertainty in the pressure shift measurements was estimated as the larger of $25 \mathrm{KHz} /$ Torr or the statistical fit uncertainty. 


$\begin{array}{ccc}\text { Temperature }(\mathrm{K}) & \gamma_{B}(\mathrm{MHz} / \mathrm{Torr}) & \gamma_{S}(T)(\mathrm{KHz} / \mathrm{Tor}) \\ 230.0 & 4.61(14) & -183(35) \\ 252.2 & 4.333(7) & -142(25) \\ 265.2 & 4.104(3) & -138(40) \\ 276.5 & 4.010(4) & -115(26) \\ 284.2 & 3.921(6) & -143(25) \\ 297.2 & 3.804(4) & -103(25) \\ 305.0 & 3.724(3) & -143(27) \\ 311.2 & 3.621(4) & -132(25) \\ 322.2 & 3.511(3) & -145(25) \\ 331.6 & 3.481(2) & -133(26)\end{array}$

Table 4.6: $183 \mathrm{GHz}$ water transition pressure broadening and pressure shift temperature dependence.

The temperature dependence of the pressure broadening coefficient $\gamma_{B}(T)$ is shown in Figure 4.41. The $\gamma_{B}(T)$ was fit to a power law, resulting in

$$
\gamma_{B}(T)=3.768(7)\left(\frac{T}{300 K}\right)^{-0.761(14)} \mathrm{MHz} / \text { Torr. }
$$




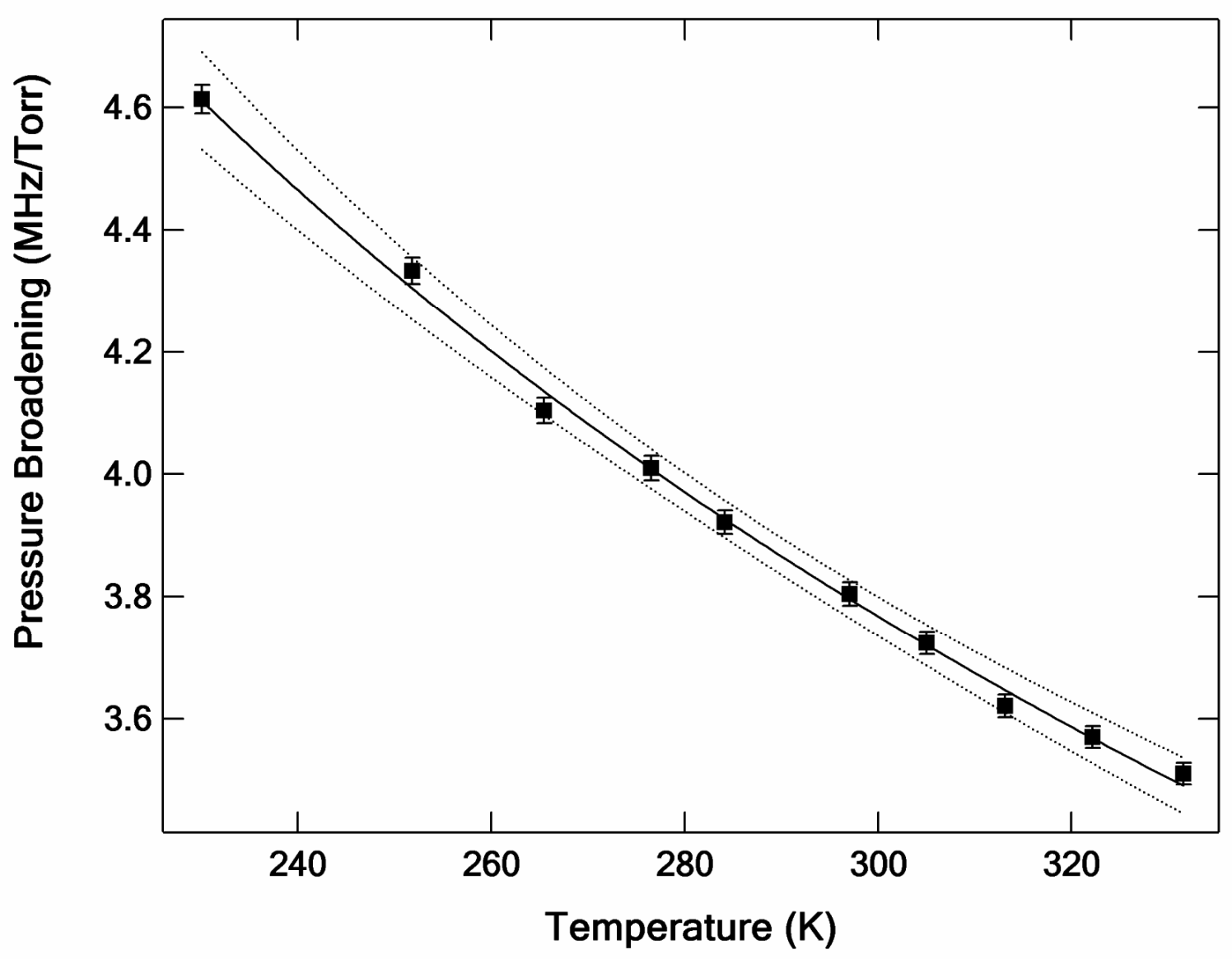

Figure 4.41: $183 \mathrm{GHz}$ water line pressure broadening coefficient $\gamma_{B}$ temperature dependence. Solid line is a fit to a power law. Dotted lines are the $3 \sigma$ confidence bands.

Our pressure broadening coefficient of $3.768(7) \mathrm{MHz} /$ Torr at $300 \mathrm{~K}$ is in good agreement with the experimentally measured values of 3.82(4) by Tretyakov et al. ${ }^{81}$, 3.87(14) by Goyette and De Lucia ${ }^{21}$ and 3.75(6) by Bauer et al. ${ }^{82}$, all recalculated for $300 \mathrm{~K}$ using the temperature exponent from equation (4.54). Our temperature dependence exponent of $-0.761(14)$ is also in agreement with $-0.76(4)$, calculated from the oxygen and nitrogen measurements by Goyette and De Lucia, ${ }^{21}$ but is higher than the value of $-0.64(10)$ by Bauer et al. ${ }^{82}$ 
The room temperature value for the pressure shift coefficient of $-103(25) \mathrm{KHz} /$ Torr is greater than $-67(16)$ measured by Tretyakov et al. ${ }^{81}$ and almost two times smaller than $-190(40)$, retrieved from the MLS data. ${ }^{83}$ It was shown theoretically by Pickett ${ }^{84}$ that the temperature exponent for the line shift $n_{S}$ is related to the temperature exponent for the pressure broadening $n_{p}$ as

$$
n_{S}=\frac{3}{2} n_{p}-\frac{1}{4}
$$

Substituting $n_{p}=-0.76$ into the above equation will give a value of -1.39 for the temperature exponent of the line shift coefficient. The temperature dependence of the line shift parameter is shown in Figure 4.42, along with the best fit of our data to a power law with a fixed -1.39 temperature exponent. The uncertainty in the pressure shift coefficient $\gamma_{S}$ measurement is comparable with the coefficient variation with temperature. This is a manifestation of the difficulty in making an accurate measurement of this small shift. 


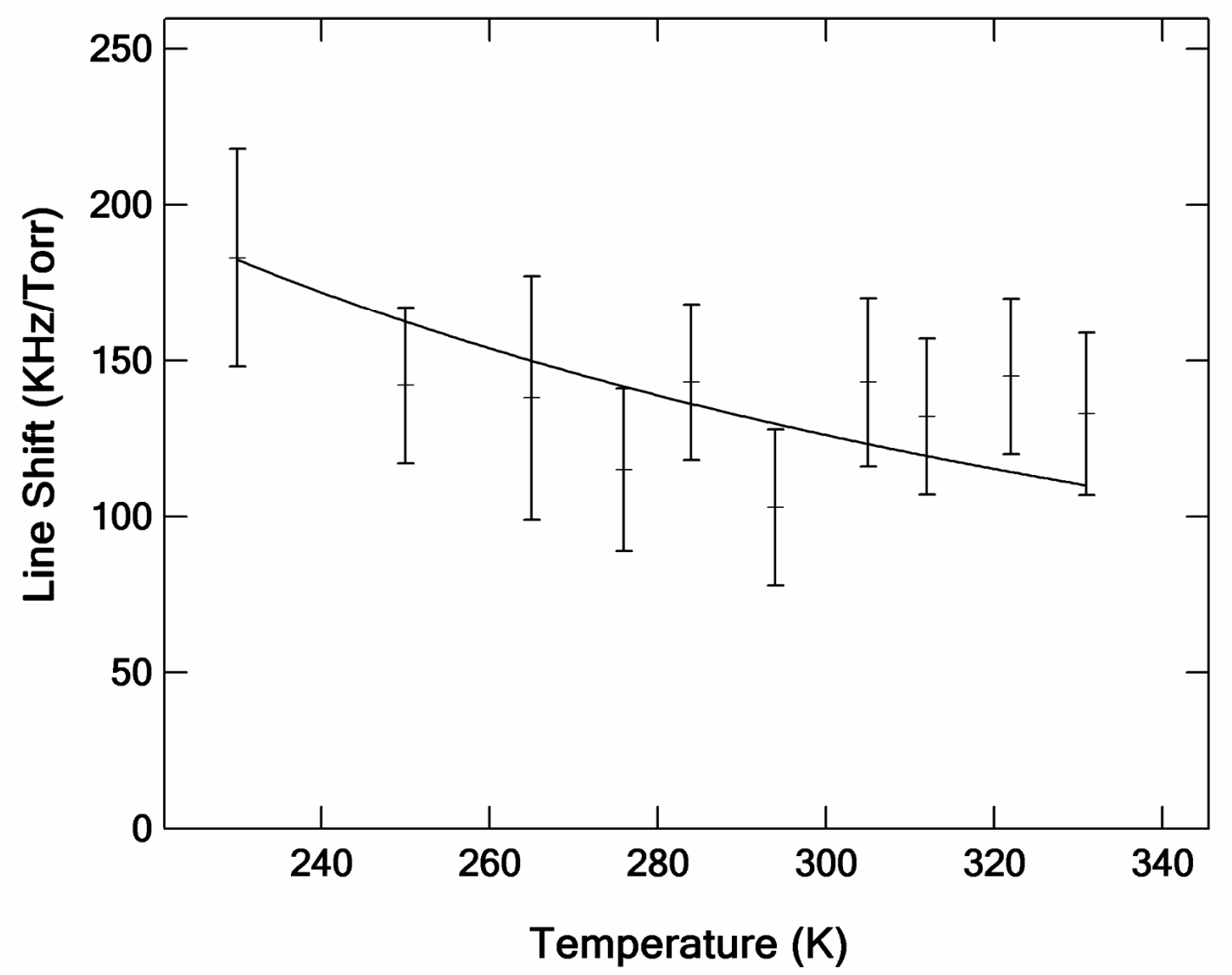

Figure 4.42: $183 \mathrm{GHz}$ water line pressure shift coefficient $\gamma_{S}$ temperature dependence. Solid line is a fit to a power law with the temperature exponent fixed at -1.39 .

\subsection{CONCLUSIONS AND FUTURE PLANS}

The time domain cavity ringdown instrument that was developed in our laboratory and described in this dissertation has many advantages when compared with other methods used to study broad, slowly changing with frequency, small absorptions. It combines the high sensitivity and great speed with the broad frequency, pressure and temperature coverage, which makes it an ideal candidate for an extensive study of the microwave atmospheric absorption. 
The sensitivity of the instrument allowed us to make the first laboratory measurements of the nitrogen microwave absorption at pressures close to atmospheric and to extensively study the dry air absorption for a wide range of temperatures and pressures in the 170-260 GHz. It was demonstrated by measurements made for the $183 \mathrm{GHz}$ water transition that the instrument can be used to study the pressure broadening and the pressure shift coefficients with great success.

Although the moist air measurements were performed with an exceptional precision, this precision and the range of these measurements were great enough to show a systematic effect that could be a result of water condensing on the resonator mirrors or the beam splitter. This non-atmospheric loss is what is currently limiting the accuracy of our moist air absorption coefficient. The addition of a third mirror that was described in Section 4.5 should make a careful study of this effect possible and present a way to measure the gas phase absorption independently of other resonator losses.

All the measurements performed so far were made in the $170-260 \mathrm{GHz}$ range of the BWO-24. The extension of the frequency range by the use of the higher frequency BWO sources is straightforward, with more measurements providing invaluable information on the atmospheric absorption.

While for many remote sensing applications, an emphasis has been on the "dry air" measurements, the physics of the oxygen and nitrogen continua are clearly very different. Further laboratory measurements, especially for the oxygen absorption temperature and frequency dependence, should provide much needed information for the theoretical community. 


\section{BIBLIOGRAPHY}

1 G. E. Becker and S. H. Autler, Phys. Rev. 70, 300 (1946).

2 Q. Ma and R. H. Tipping, J. Chem. Phys. 93(9), 6127 (1990).

3 E. Hudis, Y. Ben-Aryeh, and U. P. Oppenheim, J. Quant. Spectrosc. Rad. Transfer 47, 319 (1992).

$4 \quad$ P. W. Rosenkranz, Radio Sci. 33, 919 (1998).

5 H. A. Gebbie, W. J. Burroughs, J. E. Chamberlain, et al., Nature (London) 221(5176), 143 (1969).

6 H. A. Gebbie, J. Chem. Phys. 95(2), 1427 (1991).

7 W. B. Brown and H. A. Gebbie, Infrared Phys. 33(5), 359 (1992).

$8 \quad$ R. J. Hill, Radio Sci. 21(3), 447 (1986).

9 Q. Ma and R. H. Tipping, J. Quant. Spectrosc. Rad. Transfer 82, 517 (2003).

10 S. A. Clough, F. X. Kneizys, R. Davies, et al., in Atmsopheric Water Vapor, edited by A. Deepak, T. D. Wilkerson and L. H. Ruhnke (Academic Press, New York, 1980).

11 L. Frenkel and D. Woods, Proc. IEEE 54, 498 (1966).

12 H. J. Liebe, in Atmospheric Water Vapor, edited by A. Deepak, T. D. Wilkerson and L. H. Ruhnke (Academic Press, New York, 1980).

13 I. R. Dagg, A. Anderson, S. Yan, et al., Can. J. Phys. 63, 625 (1984). 
14 M. Godon, A. Carlier, and A. Bauer, J. Quant. Spectrosc. Radiat. Transfer 47, 275 (1991).

15 A. F. Krupnov, M. Y. Tretyakov, V. V. Parshin, et al., Journal of Molecular Spectroscopy 202, 107 (2000).

16 S. Buhler and P. Erickson, Atmospheric Millimeter and Sub-Millimeter Wave Radiative Transfer Modelling (Shaker Verlag, Aachen[Germany] ) (2000).

17 H. M. Pickett, R.L.Poynter, E. A. Cohen, et al., J.Quant. Spectrosc. Radiat. Transfer 60, 883 (1998).

18 L. S. Rothman, R. R. Gamache, R. H. Tipping, et al., J.Quant. Spectrosc. Radiat. Transfer 48, 469 (1992).

19 C. H. Townes and A. L. Schawlow, Microwave Spectroscopy, (Dover Publications, inc., New York, 1975).

20 J. H. V. Vleck and V. F. Weisskopf, Rev. Mod. Phys. 17, 227 (1945).

21 T. M. Goyette and F. C. D. Lucia, Journal of Molecular Spectroscopy 143, 346 (1990).

22 J. H. V. Vleck, Physical Review 71, 425 (1947).

23 B. Edlen, The refractive index of air, Metrologia 2 (1966) 71-80.

24 J. D. Poll and J. L. Hunt, Can. J. Phys. 54, 461 (1976).

25 N. W. Stone, L. A. A. Read, A. Anderson, et al., Can. J. Phys. 62, 338 (1984).

26 A. Borysow and L. Frommhold, The Astrophysicall Journal 311, 1043 (1986).

27 J. Boissoles, C. Boulet, R. H. Tipping, et al., J.Quant. Spectrosc. Radiat. Transfer 82, 505 (2003).

28 H. J. Liebe, Int. J. Infrared Millimeter Waves 10, 631 (1989). 
29 P. W. Rosenkranz, in Atmospheric Remote Sensing by Microwave radiometry, edited by M. A. Janssen, Chapter 2 ( John Wiley \& Sons, Inc, New York, 1993).

30 P. W. Rosenkranz, IEEE Transactions on. Antennas and Propagation AP23, 498 (1975).

31 J. H. V. Vleck, Physical Review 71, 413 (1947).

32 P. Debye, Polar Molecules, (Chemical Catalog Company, New York, 1929).

33 G. K. Hartman, Phys. Chem. Earth 25, 189 (2000).

34 H. J. Liebe, in Atmospheric Water Vapor, edited by A. Deepak, T. D. Wilkerson and L. H. Ruhnke, 143-203, (Academic Press, New York, 1980).

35 H. J. Liebe, Radio Science 20, 1069 (1985).

36 H. J. Liebe, International Journal of Infrared and Millimeter Waves 5, 207 (1984).

37 H. J. Liebe, G. A. Hufford, and M. G. Cotton, Propagation Modelling of Moist Air and Suspended Water/Ice Particles at Frequencies Below $1000 \mathrm{GHz}$, Proc. NATO/AGARD Wave Propagation Panel, 52nd Meeting, No. 3/1-10, Palma de Mallorca, Spain, 17-20 May 1993 (1993).

38 H. J. Liebe, P. W. Rosenkranz, and G. A. Hufford, J. Quant. Spectrosc. Radiat. Transfer 48, 629 (1992).

39 P. W. Rosenkranz, Radio Science 33, 919 (1998).

40 T. R. Dyke, K. M. Mack, and J. S. Muenter, J. Chem. Phys. 66, 498 (1977).

41 N. Goldman, R. S. Fellers, C. Leforestier, et al., J. Phys. Chem 105 (2001).

42 N. Goldman, C. Leforestier, and R. J. Saykally, J. Phys. Chem 108, 787 (2004).

43 H. A. Gebbie, W. J. Burroughs, J. Chamberlin, et al., Nature (London) 221, 143 (1969). 
45 S. A. Clough, F. X. Kneizys, R. Davies, et al., in Atmospheric Water Vapor, edited by A. Deepak, T. D. Wilkerson and L. H. Ruhnke, 25-47, (Academic Press, New York, 1980).

46 Q. Ma and R. H. Tipping, J. Chem. Phys. 111, 5909 (1999).

47 Q. Ma and R. H. Tipping, J. Chem. Phys. 112, 574 (2000).

48 Q. Ma and R. H. Tipping, J. Chem. Phys. 116, 4102 (2002).

49 Q. Ma and R. H. Tipping, J. Chem. Phys. 117, 10581 (2002).

50 J. H. V. Vleck and D. L. Huber, Reviews of Modern Physics 49, 939 (1977).

51 A. F. Krupnov, M. Y. Tretyakov, V. V. Parshin, et al., J. Mol. Spectrosc. 202, 107 (2000).

52 A. J. Gatesman, R. H. Giles, and J. Waldman, J. Opt. Soc. Am. B 12, 221 (1995).

53 J. D. Cook, J. W. Zwart, K. J. Long, et al., Rev. Sci. Instrum. 52, 2480 (1991).

54 A. Yariv, Introduction to optical electronics ( New York : Holt, Rinehart, and Winston, c1985).

55 P. F. Goldsmith, Quasioptical Systems (IEEE Press, c1998).

56 D. E. McCumber, Bell Syst. Tech. J. 44, 333 (1965).

57 D. E. McCumber, Bell Syst. Tech. J. 48, 1919 (1969).

58 G. T. McNice and V. E. Derr, IEEE J. Quantum Electron. QE-5, 569 (1969).

59 F. Sobel, F. L. Wentworth, and J. C. Wiltse, IRE Trans. Microwave Theory Tech. MTT-9, 512 (1961). 
60 P. A. R. Ade, J. Acres, and W. R. V. d. Reijden, Infrared Phys. 11, 233 (1971).

61 J. Poirson, F. Bretenaker, M. Vallet, et al., J. Opt. Soc. Am. B 14, 2811 (1997).

62 D. T. Petkie, T. M. Goyette, R. P. A. Bettens, et al., Rev. Sci. Instrum. 68, 1675 (1997).

63 J. W. Lamb, International Journal of Infrared and Millimeter Waves 17, 1997 (1996).

64 F. J. Tischer, IEEE Trans. Microwave Theory Tech. MTT-24, 853 (1976).

65 S. Albert, D. T. Petkie, R. P. A. Bettens, et al., Anal. Chem. 70, 719A (1998).

66 A. C. Newell and R. C. Baird, J. Appl. Phys. 36(12) (1965).

67 T. W. Pape, F. C. De Lucia, and D. D. Skatrud, J. Chem. Phys. 100, 5566 (1994).

68 G. Birnbaum and A. A. Maryott, J. Chem. Phys. 36, 2032 (1962).

69 W. Ho, I. A. Kaufman, and P. Thaddeus, J. Chem. Phys. 49, 3627 (1968).

70 I. R. Dagg, G. E. Reesor, and J. L. Urbaniak, Can. J. Phys. 53, 1764 (1975).

71 I. R. Dagg, G. E. Reesor, and M. Wong, Can. J. Phys. 56, 1037 (1978).

72 R. Occelli, H. Chaaban, J. M. Moynault, et al., Can. J. Phys. 69, 1264 (1991).

73 P. W. Rosenkranz, J. Quant. Spectrosc. Radiat. Transfer 39, 287 (1988).

74 M. Y. Tretyakov, M. A. Koshelev, V. V. Dorovskikh, et al., Journal of Molecular Spectroscopy 231, 1-14 (2005).

75 G. Y. Golubiatnikov, M. A. Koshelev, and A. F. Krupnov, Journal of Molecular Spectroscopy 222 (2003). 
76 M. Y. Tretyakov, G. Y. Golubiatnikov, V. V. Parshin, et al., Journal of Molecular Spectroscopy 223, 31 (2004).

77 D. R. Bosomworth and H. P. Gush, Can. J. Phys. 42, 751 (1965).

78 A. A. Vigasin, Journal of Molecular Spectroscopy 224, 185-187 (2004).

79 J. R. Pardo, E. Serabyn, and J. Cernicharo, Journal of Quantitative Spectroscopy \& Radiative Transfer 68, 419 (2001).

80 D. R. Bosomworth and H. P. Gush, Can. J. Phys. 43, 751 (1965).

81 M. Y. Tretyakov, V. V. Parshin, M. A. Koshelev, et al., J. Mol. Spectrosc. 218, 239 (2003).

82 A. Bauer, M. Gordon, M. Kheddar, et al., J. Quant. Spectrosc. Rad. Transfer 41, 49 (1989).

83 H. C. Pumphrey and S. Buhler, J. Quant. Spectrosc. Rad. Transfer 64, 421 (2000).

84 H. M. Pickett, J. Chem. Phys. 73, 6090 (1980). 